\title{
Finite-width effects in three-body $B$ decays
}

\author{
Hai-Yang Cheng, ${ }^{1}$ Cheng-Wei Chiang, ${ }^{2,3}$ and Chun-Khiang Chua $\odot^{4}$ \\ ${ }^{1}$ Institute of Physics, Academia Sinica, Taipei, Taiwan 115, Republic of China \\ ${ }^{2}$ Department of Physics, National Taiwan University, Taipei, Taiwan 106, Republic of China \\ ${ }^{3}$ Physics Division, National Center for Theoretical Sciences, Taipei, Taiwan 106, Republic of China \\ ${ }^{4}$ Department of Physics and Center for High Energy Physics, Chung Yuan Christian University, \\ Chung-Li, Taiwan 320, Republic of China
}

(Received 17 November 2020; accepted 12 January 2021; published 26 February 2021)

It is customary to apply the so-called narrow width approximation $\Gamma\left(B \rightarrow R P_{3} \rightarrow P_{1} P_{2} P_{3}\right)=$ $\Gamma\left(B \rightarrow R P_{3}\right) \mathcal{B}\left(R \rightarrow P_{1} P_{2}\right)$ to extract the branching fraction of the quasi-two-body decay $B \rightarrow R P_{3}$, with $R$ and $P_{3}$ being an intermediate resonant state and a pseudoscalar meson, respectively. However, the above factorization is valid only in the zero width limit. We consider a correction parameter $\eta_{R}$ from finite-width effects. Our main results are as follows: (i) We present a general framework for computing $\eta_{R}$ and show that it can be expressed in terms of the normalized differential rate and determined by its value at the resonance. (ii) We introduce a form factor $F\left(s_{12}, m_{R}\right)$ for the strong coupling involved in the $R\left(m_{12}\right) \rightarrow P_{1} P_{2}$ decay when $m_{12}$ is away from $m_{R}$. We find that off-shell effects are small in vector meson productions, but prominent in the $K_{2}^{*}(1430), \sigma / f_{0}(500)$, and $K_{0}^{*}(1430)$ resonances. (iii) We evaluate $\eta_{R}$ in the theoretical framework of QCD factorization (QCDF) and in the experimental parametrization (EXPP) for three-body decay amplitudes. In general, $\eta_{R}^{\mathrm{QCDF}}$ and $\eta_{R}^{\mathrm{EXPP}}$ are similar for vector mesons, but different for tensor and scalar resonances. A study of the differential rates enables us to understand the origin of their differences. (iv) Finite-width corrections to $\mathcal{B}\left(B^{-} \rightarrow R P\right)_{\mathrm{NWA}}$ obtained in the narrow width approximation are generally small, less than $10 \%$, but they are prominent in $B^{-} \rightarrow \sigma / f_{0}(500) \pi^{-}$and $B^{-} \rightarrow \bar{K}_{0}^{* 0}(1430) \pi^{-}$decays. The EXPP of the normalized differential rates should be contrasted with the theoretical predictions from QCDF calculation as the latter properly takes into account the energy dependence in weak decay amplitudes. (v) It is common to use the Gounaris-Sakurai model to describe the line shape of the broad $\rho(770)$ resonance. After including finite-width effects, the PDG value of $\mathcal{B}\left(B^{-} \rightarrow \rho \pi^{-}\right)=(8.3 \pm 1.2) \times 10^{-6}$ should be corrected to $(7.9 \pm 1.1) \times 10^{-6}$ in EXPP and $(7.7 \pm 1.1) \times 10^{-6}$ in QCDF. (vi) For the very broad $\sigma / f_{0}(500)$ scalar resonance, we use a simple pole model to describe its line shape and find a very large width effect: $\eta_{\sigma}^{\text {QCDF }} \sim$ 2.15 and $\eta_{\sigma}^{\mathrm{EXPP}} \sim 1$ 1.64. Consequently, $B^{-} \rightarrow \sigma \pi^{-}$has a large branching fraction of order $10^{-5}$. (vii) We employ the Breit-Wigner line shape to describe the production of $K_{0}^{*}(1430)$ in three-body $B$ decays and find large off-shell effects. The smallness of $\eta_{K_{0}^{*}}^{\mathrm{QCDF}}$ relative to $\eta_{K_{0}^{*}}^{\mathrm{EXP}}$ is ascribed to the differences in the normalized differential rates off the resonance. (viii) In the approach of QCDF, the calculated $C P$ asymmetries of $B^{-} \rightarrow f_{2}(1270) \pi^{-}, \sigma / f_{0}(500) \pi^{-}, K^{-} \rho^{0}$ decays agree with the experimental observations. The nonobservation of $C P$ asymmetry in $B^{-} \rightarrow \rho(770) \pi^{-}$can also be accommodated in QCDF.

DOI: 10.1103/PhysRevD.103.036017

\section{INTRODUCTION}

In a three-body decay with resonance contributions, it is a common practice to apply the factorization relation, also known as the narrow width approximation (NWA), to factorize the process as a quasi-two-body weak decay

Published by the American Physical Society under the terms of the Creative Commons Attribution 4.0 International license. Further distribution of this work must maintain attribution to the author(s) and the published article's title, journal citation, and DOI. Funded by SCOAP . followed by another two-body strong decay. Take a $B$ meson decay $B \rightarrow R P_{3} \rightarrow P_{1} P_{2} P_{3}$ as an example, where $R$ and $P_{3}$ are an intermediate resonant state and a pseudoscalar meson, respectively. One then uses

$\Gamma\left(B \rightarrow R P_{3} \rightarrow P_{1} P_{2} P_{3}\right)=\Gamma\left(B \rightarrow R P_{3}\right) \mathcal{B}\left(R \rightarrow P_{1} P_{2}\right)$

to extract the branching fraction of the quasi-two-body decay, $\mathcal{B}\left(B \rightarrow R P_{3}\right)$, which is then compared with theoretical predictions. However, such an approach is valid only in the narrow width limit, $\Gamma_{R} \rightarrow 0$. In other words, one should have instead 
$\Gamma\left(B \rightarrow R P_{3} \rightarrow P_{1} P_{2} P_{3}\right)_{\Gamma_{R} \rightarrow 0}=\Gamma\left(B \rightarrow R P_{3}\right) \mathcal{B}\left(R \rightarrow P_{1} P_{2}\right)$,

where we have assumed that both $\Gamma\left(B \rightarrow R P_{3}\right)$ and $\mathcal{B}\left(R \rightarrow P_{1} P_{2}\right)$ are not affected by the NWA. In other words, while taking the $\Gamma\left(R \rightarrow P_{1} P_{2}\right) \rightarrow 0$ limit, the branching fraction of $R \rightarrow P_{1} P_{2}$ is assumed to remain intact. For the case when $R$ has a finite width, Eq. (1.1) does not hold. Moreover, theoretical predictions of $\mathcal{B}\left(B \rightarrow R P_{3}\right)$ are normally calculated under the assumption that the both final-state particles are stable (i.e., $\Gamma_{R}, \Gamma_{P_{3}} \rightarrow 0$ ). Therefore, the question is how one should extract $\mathcal{B}\left(B \rightarrow R P_{3}\right)$ from the experimental measurement of the partial rate of $B \rightarrow$ $R P_{3} \rightarrow P_{1} P_{2} P_{3}$ and make a meaningful comparison with its theoretical predictions.

Let us define a quantity ${ }^{1}$

$$
\begin{aligned}
\eta_{R} & \equiv \frac{\Gamma\left(B \rightarrow R P_{3} \rightarrow P_{1} P_{2} P_{3}\right)_{\Gamma_{R} \rightarrow 0}}{\Gamma\left(B \rightarrow R P_{3} \rightarrow P_{1} P_{2} P_{3}\right)} \\
& =\frac{\Gamma\left(B \rightarrow R P_{3}\right) \mathcal{B}\left(R \rightarrow P_{1} P_{2}\right)}{\Gamma\left(B \rightarrow R P_{3} \rightarrow P_{1} P_{2} P_{3}\right)}=1+\delta,
\end{aligned}
$$

so that the deviation of $\eta_{R}$ from unity measures the degree of departure from the NWA when the width is finite. It is naively expected that the correction $\delta$ will be of order $\Gamma_{R} / m_{R}$. The quantity $\eta_{R}$ extrapolates the three-body decay from the physical width to the zero width. It is calculable theoretically but depends on the line shape of the resonance and the approach of describing weak hadronic decays such as QCD factorization (QCDF), perturbative QCD, and soft collinear effective theory. After taking into account the finite-width effect $\eta_{R}$ from the resonance, the branching fraction of the quasi-two-body decay reads

$\mathcal{B}\left(B \rightarrow R P_{3}\right)=\eta_{R} \frac{\mathcal{B}\left(B \rightarrow R P_{3} \rightarrow P_{1} P_{2} P_{3}\right)_{\mathrm{expt}}}{\mathcal{B}\left(R \rightarrow P_{1} P_{2}\right)_{\text {expt }}}$.

Note that $\mathcal{B}\left(B \rightarrow R P_{3}\right)$ on the left-hand side of the above formula is the branching fraction under the assumption that both $R$ and $P_{3}$ are stable and thus have zero decay width. Therefore, it is suitable for a comparison with theoretical calculations.

In the literature, such as the Particle Data Group (PDG) [3], the branching fraction of the quasi-two-body decay is often inferred from Eq. (1.4) by setting $\eta_{R}$ equal to unity. While this is justified for narrow-width resonances, it is not so for the broad ones. For example, $\Gamma_{\rho} / m_{\rho}=0.192$ for the $\rho$ vector meson, $\Gamma_{f_{2}} / m_{f_{2}}=0.146$ for the $f_{2}(1270)$ tensor

\footnotetext{
${ }^{1}$ For later convenience, our definition of $\eta_{R}$ here is inverse to the one defined in [1]. A similar (but inversely) quantity $\mathcal{W}_{R}^{(\ell)}=$ $\Gamma_{R}^{(\ell)} / \Gamma_{R, \mathrm{NWL}}^{(\ell)}$ was also considered in [2], where $\Gamma_{R}^{(\ell)}$ is the partialwave decay rate integrated in a region around a resonance and $\Gamma_{R, \mathrm{NWL}}^{(\ell)}$ denotes $\Gamma_{R}^{(\ell)}$ in the narrow width limit.
}

meson, $\Gamma_{\sigma} / m_{\sigma} \sim \mathcal{O}(1)$ for the $\sigma / f_{0}(500)$ scalar meson, and $\Gamma_{K_{2}^{*}} / m_{K_{2}^{*}} \approx 0.189$ for the $K_{2}^{*}(1430)$ tensor meson. For these resonances, finite-width effects seem to be important and cannot be neglected. We shall see in this work that the deviation of $\eta_{R}$ from unity does not always follow the guideline from the magnitude of $\Gamma_{R} / m_{R}$.

It is worth mentioning that the finite-width effects play an essential role in charmed meson decays $[1,4]$. There exist some modes, e.g., $D^{0} \rightarrow \rho(1700)^{+} K^{-}, D^{0} \rightarrow$ $K^{*}(1410)^{-} K^{+}$which are not allowed kinematically can proceed through the finite-width effects.

In this work, we will calculate the parameter $\eta_{R}$ within the framework of QCDF for various resonances and use these examples to highlight the importance of finite-width effects. First, we need to check the NWA relation Eq. (1.2) both analytically and numerically. Once this is done, it is straightforward to compute $\eta_{R}$.

In the experimental analysis of $B \rightarrow R P_{3} \rightarrow P_{1} P_{2} P_{3}$ decays, it is customary to parametrize the amplitude as $A\left(m_{12}, m_{23}\right)=c F\left(m_{12}, m_{23}\right)$, where the strong dynamics is described by the function $F$ that parametrizes the intermediate resonant processes, while the information of weak interactions is encoded in the complex coefficient $c$ which is obtained by fitting to the measured Dalitz plot. The function $F$ can be further parametrized in terms of a resonance line shape, an angular dependence, and BlattWeisskopf barrier factors. Using the experimental parametrization of $F\left(m_{12}, m_{23}\right)$, we can also compute the ratio of the three-body decay rate without and with the finitewidth effects of the resonance, which we shall refer to as $\eta_{R}^{\mathrm{EXPP}}$. Obviously, $\eta_{R}^{\mathrm{EXPP}}$ is independent of $c$. On the contrary, the weak decay amplitude of $B \rightarrow R\left(m_{12}\right) P_{3}$ generally has some dependence on $m_{12}$ in QCDF calculations. Hence, $\eta_{R}^{\mathrm{QCDF}}$ is different from $\eta_{R}^{\mathrm{EXPP}}$ in general. It will be instructive to compare them to gain more insight to the underlying mechanism.

Although it is straightforward to estimate the parameter $\eta_{R}$ in a theoretical framework by computing the decay rates of the quasi-two-body decay and the corresponding threebody decay, we shall develop a general framework for the study of $\eta_{R}$. We will show that $\eta_{R}$ can be expressed in terms of a normalized differential decay rate. It turns out that $\eta_{R}$ is nothing but the value of the normalized differential decay rate evaluated at the contributing resonance. Not only is the calculation significantly simplified, the underlying physics also becomes more transparent. Finally, we note in passing that while we focus on three-body $B$ meson decays in this paper to elucidate our point and explain the cause, our finding generally applies to all quasi-two-body decays.

The layout of the present paper is as follows. In Sec. II, we present a general framework for the study of the parameter $\eta_{R}$ and show that it can be obtained from the normalized differential decay rate. The experimental analysis of $B \rightarrow R P_{3} \rightarrow P_{1} P_{2} P_{3}$ decays relies on a parametrization of the involved strong dynamics. This is discussed in detail 
in Sec. III. We then proceed to evaluate $\eta_{R}^{\mathrm{QCDF}}$ within the framework of QCDF in Sec. IV for some selected processes mediated by tensor, vector and scalar resonances, and compare them with $\eta_{R}^{\text {EXPP }}$ determined from the experimental parametrization. We discuss our findings in Sec. V. Section VI comes to our conclusions. A more concise version of this work has been presented in [5].

\section{GENERAL FRAMEWORK}

In this section, we discuss how $\eta_{R}$ can be determined from a normalized differential decay rate. We start by considering the simpler case where the mediating resonance is a scalar meson and show that the result reduces to the usual one in the NWA. We then generalize our discussions to resonances of arbitrary spin and derive an important relation between $\eta_{R}$ and the normalized differential decay rate evaluated at the resonance mass. Two examples of the $\rho(770)$ and $\sigma / f_{0}(500)$ resonances are presented at the end of the section.

\section{A. Scalar intermediate states}

We first consider the case that $R$ is a scalar resonance for simplicity. The three-body $B \rightarrow R P_{3} \rightarrow P_{1} P_{2} P_{3}$ decay amplitude has the following form:

$A\left(m_{12}, m_{23}\right)=\frac{\mathcal{M}\left[B \rightarrow R\left(m_{12}\right) P_{3}\right] \mathcal{M}\left[R\left(m_{12}\right) \rightarrow P_{1} P_{2}\right]}{\left(m_{12}^{2}-m_{R}^{2}\right)+i m_{R} \Gamma_{R}}$,

where $\mathcal{M}\left[B \rightarrow R\left(m_{12}\right) P_{3}\right]$ and $\mathcal{M}\left[R\left(m_{12}\right) \rightarrow P_{1} P_{2}\right]$ are weak and strong decay amplitudes of $B \rightarrow R\left(m_{12}\right) P_{3}$ and $R\left(m_{12}\right) \rightarrow P_{1} P_{2}$ decays, respectively, and $m_{i j}^{2} \equiv p_{i j}^{2} \equiv$ $\left(p_{i}+p_{j}\right)^{2}$. Note that at the resonance, we have

$$
\begin{aligned}
& i \sqrt{\pi m_{R} \Gamma_{R}} A\left(m_{R}, m_{23}\right) \\
& \quad=\mathcal{M}\left[B \rightarrow R\left(m_{R}\right) P_{3}\right] \frac{\mathcal{M}\left[R\left(m_{R}\right) \rightarrow P_{1} P_{2}\right]}{\sqrt{m_{R} \Gamma_{R} / \pi}},
\end{aligned}
$$

which contains the critical information of the physical $B \rightarrow$ $R P_{3}$ and $R \rightarrow P_{1} P_{2}$ decay amplitudes.

Using the standard formulas [3], the three-body differential decay rate at the resonance is given by

$$
\frac{d \Gamma\left(m_{R}^{2}\right)}{d m_{12}^{2}}=\frac{1}{(2 \pi)^{3}} \frac{1}{32 m_{B}^{3}} \int\left|A\left(m_{R}, m_{23}\right)\right|^{2} d m_{23}^{2},
$$

or equivalently,

$$
\begin{aligned}
\frac{d \Gamma\left(m_{R}^{2}\right)}{d m_{12}^{2}}= & \frac{1}{(2 \pi)^{5}} \frac{1}{32 m_{R} m_{B}^{2}} \\
& \times \int\left|A\left(m_{R}, m_{23}\right)\right|^{2}\left|\vec{p}_{1}\right|\left|\vec{p}_{3}\right| d \Omega_{1} d \Omega_{3},
\end{aligned}
$$

where $\left|\vec{p}_{1}\right|$ and $\Omega_{1}$ are evaluated in the $R$ rest frame. With the help of Eq. (2.2), the above equation can be rewritten as

$$
\begin{aligned}
& \pi m_{R} \Gamma_{R} \frac{d \Gamma\left(m_{R}^{2}\right)}{d m_{12}^{2}} \\
= & \frac{1}{32 \pi^{2}} \int\left|\mathcal{M}\left[B \rightarrow R\left(m_{R}\right) P_{3}\right]\right|^{2} \frac{\left|\vec{p}_{3}\right|}{m_{B}^{2}} d \Omega_{3} \\
& \times\left(\frac{1}{32 \pi^{2}} \int\left|\mathcal{M}\left[R\left(m_{R}\right) \rightarrow P_{1} P_{2}\right]\right|^{2} \frac{\left|\vec{p}_{1}\right|}{m_{R}^{2}} d \Omega_{1}\right) / \Gamma_{R}, \\
= & \Gamma\left(B \rightarrow R P_{3}\right) \mathcal{B}\left(R \rightarrow P_{1} P_{2}\right) .
\end{aligned}
$$

Hence, we obtain

$$
\begin{aligned}
& \Gamma\left(B \rightarrow R P_{3}\right) \mathcal{B}\left(R \rightarrow P_{1} P_{2}\right) \\
& =\pi m_{R} \Gamma_{R} \frac{d \Gamma\left(m_{R}^{2}\right)}{d m_{12}^{2}} \\
& =\frac{\pi m_{R} \Gamma_{R}}{(2 \pi)^{3}} \frac{1}{32 m_{B}^{3}} \int_{\left(m_{23}^{2}\right) \min \left(m_{R}\right)}^{\left(m_{23}^{2}\right)_{\max }\left(m_{R}\right)}\left|A\left(m_{R}, m_{23}\right)\right|^{2} d m_{23}^{2} .
\end{aligned}
$$

Consequently, Eqs. (2.6) and (1.3) imply that $\eta_{R}$ is related to the normalized differential rate,

$\eta_{R}=\frac{\pi m_{R} \Gamma_{R} \frac{d \Gamma\left(m_{R}^{2}\right)}{d m_{12}^{2}}}{\int \frac{d \Gamma\left(m_{12}^{2}\right)}{d m_{12}^{2}} d m_{12}^{2}}=\pi m_{R} \Gamma_{R} \frac{\int\left|A\left(m_{R}, m_{23}\right)\right|^{2} d m_{23}^{2}}{\int\left|A\left(m_{12}, m_{23}\right)\right|^{2} d m_{12}^{2} d m_{23}^{2}}$.

With the help of the following identity ${ }^{2}$ :

$$
\lim _{\Gamma_{R} \rightarrow 0} \frac{m_{R} \Gamma_{R} / \pi}{\left(m_{12}^{2}-m_{R}^{2}\right)^{2}+m_{R}^{2} \Gamma_{R}^{2}}=\delta\left(m_{12}^{2}-m_{R}^{2}\right),
$$

one can readily verify that $\eta_{R}$ given in the above equation approaches unity in the narrow width limit, reproducing the well-known result of Eq. (1.2).

\section{B. General case}

Although Eqs. (2.6) and (2.7) are derived for the case of a scalar resonance, they can be generalized to a more generic case, where the resonance particle has spin $J$. Instead of Eq. (2.1), the general amplitude has the following expression:

$$
A\left(m_{12}, m_{23}\right)=\mathcal{M}\left(m_{12}, m_{12}\right) R_{J}\left(m_{12}\right) \mathcal{T}_{J}\left(m_{12}, m_{23}\right),
$$

where $\mathcal{M}\left(m_{12}, m_{12}\right)$ is a regular function containing the information of $B \rightarrow R\left(m_{12}\right) P_{3}$ weak decay and $R\left(m_{12}\right) \rightarrow$ $P_{1} P_{2}$ strong decay, $R_{J}$ describes the line shape of the resonance, and $\mathcal{T}_{J}$ encodes the angular dependence.

\footnotetext{
${ }^{2}$ This follows from the formula $\lim _{\epsilon \rightarrow 0} \frac{\epsilon}{\epsilon^{2}+x^{2}}=\pi \delta(x)$.
} 
Resonant contributions are commonly depicted by the relativistic Breit-Wigner (BW) line shape,

$$
R_{J}^{\mathrm{BW}}\left(m_{12}\right)=\frac{1}{\left(m_{12}^{2}-m_{R}^{2}\right)+i m_{R} \Gamma_{R}\left(m_{12}\right)} .
$$

In general, the mass-dependent width is expressed as

$$
\Gamma_{R}\left(m_{12}\right)=\Gamma_{R}^{0}\left(\frac{q}{q_{0}}\right)^{2 J+1} \frac{m_{R}}{m_{12}} \frac{X_{J}^{2}(q)}{X_{J}^{2}\left(q_{0}\right)},
$$

where $q=\left|\vec{p}_{1}\right|=\left|\vec{p}_{2}\right|$ is the center-of-mass (c.m.) momentum in the rest frame of the resonance $R, q_{0}$ is the value of $q$ when $m_{12}$ is equal to the pole mass $m_{R}$, and $X_{J}$ is a BlattWeisskopf barrier factor given by

$$
\begin{aligned}
& X_{0}(z)=1, \quad X_{1}(z)=\sqrt{\frac{1}{\left(z r_{\mathrm{BW}}\right)^{2}+1}}, \\
& X_{2}(z)=\sqrt{\frac{1}{\left(z r_{\mathrm{BW}}\right)^{4}+3\left(z r_{\mathrm{BW}}\right)^{2}+9}},
\end{aligned}
$$

with $r_{\mathrm{BW}} \approx 4.0 \mathrm{GeV}^{-1}$. In Eq. (2.11), $\Gamma_{R}^{0}$ is the nominal total width of $R$ with $\Gamma_{R}^{0}=\Gamma_{R}\left(m_{R}\right)$. One advantage of using the energy-dependent decay width is that $\Gamma_{R}\left(m_{12}\right)$ vanishes when $m_{12}$ is below the $m_{1}+m_{2}$ threshold [see the expression of $q$ in Eq. (3.9) below]. Hence, the factor $q^{2 L+1}$ with $L$ being the orbital angular momentum between $R$ and $P_{3}$ guarantees the correct threshold behavior. The rapid growth of this factor for angular momenta $>L$ is compensated at higher energies by the Blatt-Weisskopf barrier factors [3].

From Eqs. (3.8), (3.10), (4.46), (4.61), and (4.102) below, we find that the angular distribution term $\mathcal{T}_{J}$ in Eq. (2.9) at the resonance is governed by the Legendre polynomial $P_{J}(\cos \theta)$, where $\theta$ is the angle between $\vec{p}_{1}$ and $\vec{p}_{3}$ measured in the rest frame of the resonance (see also [6]). Explicitly, we have

$$
\begin{aligned}
& P_{0}(\cos \theta)=1, \quad P_{1}(\cos \theta)=\cos \theta, \\
& P_{2}(\cos \theta)=\frac{1}{2}\left(-1+3 \cos ^{2} \theta\right),
\end{aligned}
$$

and

$$
\mathcal{T}_{0}\left(m_{R}, m_{23}\right)=1, \quad \mathcal{T}_{1}\left(m_{R}, m_{23}\right) \propto \cos \theta,
$$$$
\mathcal{T}_{2}\left(m_{R}, m_{23}\right) \propto 1-3 \cos ^{2} \theta \text {. }
$$

Note that $\mathcal{T}_{0}\left(m_{12}, m_{23}\right)=1$ throughout the entire phase space. This means that the strong and weak amplitudes can always be separated for the scalar case, as shown in Eq. (2.1).

Instead of Eq. (2.2), the general amplitude at the resonance takes the form

$$
\begin{aligned}
i \sqrt{\pi m_{R} \Gamma_{R}^{0}} A\left(m_{R}, m_{23}\right)= & \sum_{\lambda} \mathcal{M}_{\lambda}\left[B \rightarrow R\left(m_{R}\right) P_{3}\right] \\
& \times \frac{\mathcal{M}_{\lambda}\left[R\left(m_{R}\right) \rightarrow P_{1} P_{2}\right]}{\sqrt{m_{R} \Gamma_{R}^{0} / \pi}},
\end{aligned}
$$

where $\lambda$ is the helicity of the resonance $R$. Such a relation is expectable because there is a propagator of the resonance $R$ in the amplitude $A\left(m_{12}, m_{23}\right)$ and its denominator reduces to $i m_{R} \Gamma_{R}^{0}$ on the mass shell of $m_{12}$ while its numerator reduces to a polarization sum of the polarization vectors, producing the above structure after contracted with the rest of the amplitude.

From Eqs. (2.4) and (2.15), we have

$$
\begin{aligned}
\frac{d \Gamma\left(m_{R}^{2}\right)}{d m_{12}^{2}}= & \frac{1}{\left(32 \pi^{2}\right)^{2} \pi m_{R}\left(\Gamma_{R}^{0}\right)^{2}} \\
& \times \int \mid \sum_{\lambda} \mathcal{M}_{\lambda}\left[B \rightarrow R\left(m_{R}\right) P_{3}\right] \mathcal{M}_{\lambda}\left[R\left(m_{R}\right)\right. \\
& \left.\rightarrow P_{1} P_{2}\right]\left.\right|^{2} \frac{\left|\vec{p}_{1}\right|}{m_{R}^{2}} \frac{\left|\vec{p}_{3}\right|}{m_{B}^{2}} d \Omega_{3} d \Omega_{1},
\end{aligned}
$$

where $\left|\vec{p}_{1}\right|$ and $\Omega_{1}$ are evaluated in the $R$ rest frame. In this frame, the sum over helicities in the amplitude can be replaced by the sum over spins. Consequently, $\mathcal{M}_{\lambda}[B \rightarrow$ $\left.R\left(m_{R}\right) P_{3}\right]$ and $\mathcal{M}_{\lambda}\left[R\left(m_{R}\right) \rightarrow P_{1} P_{2}\right]$ are proportional to $Y_{J \lambda}^{*}\left(\Omega_{3}\right)$ and $Y_{J \lambda}\left(\Omega_{1}\right)$, respectively. ${ }^{3}$ As a cross-check, we note that Eq. (2.14) can be reproduced by using the well-known addition theorem of spherical harmonics, $(2 J+1) P_{J}(\cos \theta)=4 \pi \sum_{\lambda} Y_{J \lambda}^{*}\left(\Omega_{3}\right) Y_{J \lambda}\left(\Omega_{1}\right)$. Alternatively, we can start from Eq. (2.14) and make use of the addition theorem to obtain the $\sum_{\lambda} Y_{J \lambda}^{*}\left(\Omega_{3}\right) Y_{J \lambda}\left(\Omega_{1}\right)$ factor.

We now see that the interference terms in Eq. (2.16) from different helicities (or spins) vanish after the angular integrations. As a result, we obtain

$$
\begin{aligned}
\pi & m_{R} \Gamma_{R} \frac{d \Gamma\left(m_{R}^{2}\right)}{d m_{12}^{2}} \\
= & \frac{1}{32 \pi^{2}} \sum_{\lambda} \int\left|\mathcal{M}_{\lambda}\left[B \rightarrow R\left(m_{R}\right) P_{3}\right]\right|^{2} \frac{\left|\vec{p}_{3}\right|}{m_{B}^{2}} d \Omega_{3} \\
& \times\left(\frac{1}{32 \pi^{2}} \int\left|\mathcal{M}_{\lambda}\left[R\left(m_{R}\right) \rightarrow P_{1} P_{2}\right]\right|^{2} \frac{\left|\vec{p}_{1}\right|}{m_{R}^{2}} d \Omega_{1}\right) / \Gamma_{R}^{0} \\
= & \Gamma\left(B \rightarrow R P_{3}\right) \mathcal{B}\left(R \rightarrow P_{1} P_{2}\right),
\end{aligned}
$$

where we have made use of the fact that the branching fraction $\mathcal{B}\left(R \rightarrow P_{1} P_{2}\right)$ is independent of the helicity

\footnotetext{
${ }^{3}$ For example, in the $J=1$ case and at the resonance, $\mathcal{M}_{\lambda}[B \rightarrow$ $\left.V\left(m_{R}\right) P_{3}\right]$ is proportional to $p_{B} \cdot \epsilon^{*}\left(p_{12}, \lambda\right)$, while $\mathcal{M}_{\lambda}\left[V\left(m_{R}\right) \rightarrow\right.$ $\left.P_{1} P_{2}\right]$ is proportional to $\epsilon\left(p_{12}, \lambda\right) \cdot\left(p_{1}-p_{2}\right)$. See also Eq. (3.8) below. It can be easily seen that in the $V$ rest frame, these terms provide the $Y_{1 \lambda}^{*}\left(\Omega_{3}\right)$ and $Y_{1 \lambda}\left(\Omega_{1}\right)$ factors, respectively.
} 
(or spin) in the last step. The above equation agrees with Eq. (2.6), and consequently Eq. (2.7) follows.

Equation (2.7) can be easily generalized to the case with identical particles in the final state. Let $P_{2}$ and $P_{3}$ be identical particles so that the decay amplitude reads $\mathcal{M}=A\left(m_{12}, m_{23}\right)+A\left(m_{13}, m_{23}\right)$, giving

$\eta_{R}=\pi m_{R} \Gamma_{R} \frac{2 \int\left|A\left(m_{R}, m_{23}\right)\right|^{2} d m_{23}^{2}}{\int\left|A\left(m_{12}, m_{23}\right)+A\left(m_{13}, m_{23}\right)\right|^{2} d m_{12}^{2} d m_{23}^{2}}$.

Furthermore, from Eqs. (2.8) and (2.9), we see that in the narrow width limit, the amplitude squared takes the form

$\left|A\left(m_{12}, m_{23}\right)\right|_{\Gamma_{R}^{0} \rightarrow 0}^{2}=\pi m_{R} \Gamma_{R}^{0} \delta\left(m_{12}^{2}-m_{R}^{2}\right)\left|A\left(m_{R}, m_{23}\right)\right|^{2}$.

Substituting this into Eq. (2.7), we obtain $\eta_{R}=1$ in the limit of zero width, hence reproducing the well-known result in Eq. (1.2).

In this work, we will consider $A\left(m_{12}, m_{23}\right)$ using the experimental parametrization (EXPP) and the QCDF calculation and compute $\eta_{R}^{\mathrm{EXPP}}$ and $\eta_{R}^{\mathrm{QCDF}}$, respectively. In the latter case, we shall see that in the narrow width limit, the weak interaction part of the amplitude does reduce to the QCDF amplitude of the $B \rightarrow R P_{3}$ decay. We will also show explicitly the validity of the factorization relation in the zero width limit for several selected examples of threebody decays involving tensor, vector, and scalar mediating resonances.

\section{C. $\eta_{R}$ and the normalized differential rate}

As suggested by Eq. (2.7), $\eta_{R}$ can be expressed in terms of the normalized differential rate,

$$
\eta_{R}=\pi m_{R} \Gamma_{R} \frac{d \tilde{\Gamma}\left(m_{R}^{2}\right)}{d m_{12}^{2}}=\frac{1}{2} \pi \Gamma_{R} \frac{d \tilde{\Gamma}\left(m_{R}\right)}{d m_{12}},
$$

where we have defined

$$
\frac{d \tilde{\Gamma}\left(m_{12}^{2}\right)}{d m_{12}^{2}} \equiv \frac{d \Gamma\left(m_{12}^{2}\right)}{d m_{12}^{2}} / \int \frac{d \Gamma\left(m_{12}^{2}\right)}{d m_{12}^{2}} d m_{12}^{2} .
$$

Hence, $\eta_{R}$ is determined by the value of the normalized differential rate at the resonance. It should be noted that as the normalized differential rate is always positive and normalized to 1 after integration, the value of $d \tilde{\Gamma}\left(m_{R}\right) /$ $d m_{12}$ is anticorrelated with $d \tilde{\Gamma}\left(m_{12}\right) / d m_{12}$ elsewhere. Hence, it is the shape of the (normalized) differential rate that matters in the determination of $\eta_{R}$.

The above point can be made more precise. When $\Gamma_{R} / m_{R} \ll 1$, we expect that the normalized differential rate around the resonance is reasonably well described as $\left.\frac{d \tilde{\Gamma}\left(m_{12}^{2}\right)}{d m_{12}^{2}}\right|_{m_{12}^{2} \simeq m_{R}^{2}} \simeq \frac{m_{R}^{2} \Gamma_{R}^{2}}{\left(m_{12}^{2}-m_{R}^{2}\right)^{2}+m_{R}^{2} \Gamma_{R}^{2}} \frac{d \tilde{\Gamma}\left(m_{R}^{2}\right)}{d m_{12}^{2}}$.

It is straightforward to show that as a result, Eq. (2.20) can be approximated by

$$
\eta_{R} \simeq \frac{\pi}{2 \tan ^{-1} 2} \int_{\left(m_{R}-\Gamma_{R}\right)^{2}}^{\left(m_{R}+\Gamma_{R}\right)^{2}} \frac{d \tilde{\Gamma}\left(m_{12}^{2}\right)}{d m_{12}^{2}} d m_{12}^{2},
$$

or equivalently,

$$
\begin{aligned}
\eta_{R} & \simeq \frac{\pi}{2 \tan ^{-1} 2} \int_{m_{R}-\Gamma_{R}}^{m_{R}+\Gamma_{R}} \frac{d \tilde{\Gamma}\left(m_{12}\right)}{d m_{12}} d m_{12} \\
& =\frac{\pi}{2 \tan ^{-1} 2}\left(1-\int_{\text {elsewhere }} \frac{d \tilde{\Gamma}\left(m_{12}\right)}{d m_{12}} d m_{12}\right) .
\end{aligned}
$$

It becomes clear that $\eta_{R}$ represents the fraction of rates around the resonance and is anticorrelated with the fraction of rates off the resonance.

The EXPP and the QCDF approaches may have different shapes in the differential rates, resulting in different $\eta_{R}$ 's, i.e., $\eta_{R}^{\mathrm{EXPP}} \neq \eta_{R}^{\mathrm{QCDF}}$ in general. The two-body rate reported by experiments should be corrected using $\eta_{R}=\eta_{R}^{\text {EXPP }}$ in Eq. (1.4), as the data are extracted using the experimental parametrization. On the other hand, the experimental parametrization on normalized differential rates should be compared with the theoretical predictions from QCDF calculation as the latter takes into account the energy dependence of weak interaction amplitudes. As we shall show in Sec. V.A, the usual experimental parametrization ignores the momentum dependence in weak dynamics and would lead to incorrect extraction of quasi-two-body decay rates in the case of broad resonances, as contrasted with the estimates using the QCDF approach.

\section{Formula of $\eta_{R}$ in the case of the Gounaris-Sakurai line shape}

A popular choice for describing the broad $\rho(770)$ resonance is the Gounaris-Sakurai (GS) model [7]. It was employed by both $B A B A R$ [8] and LHCb $[9,10]$ Collaborations in their analyses of the $\rho(770)$ resonance in the $B^{-} \rightarrow \pi^{+} \pi^{-} \pi^{-}$decay. The GS line shape for $\rho(770)$ is given by

$$
T_{\rho}^{\mathrm{GS}}(s)=\frac{1+D \Gamma_{\rho}^{0} / m_{\rho}}{s-m_{\rho}^{2}-f(s)+i m_{\rho} \Gamma_{\rho}(s)},
$$

where

$$
\Gamma_{\rho}(s)=\Gamma_{\rho}^{0}\left(\frac{q}{q_{0}}\right)^{3} \frac{m_{\rho}}{\sqrt{s}} \frac{X_{1}^{2}(q)}{X_{1}^{2}\left(q_{0}\right)},
$$

the Blatt-Weisskopf barrier factor is given in Eq. (2.12), $\Gamma_{\rho}^{0}$ is the nominal total $\rho$ width with $\Gamma_{\rho}^{0}=\Gamma_{\rho}\left(m_{\rho}^{2}\right)$. The quantities $q$ and $q_{0}$ are already introduced before in 
Sec. II. A. In this model, the real part of the pion-pion scattering amplitude with an intermediate $\rho$ exchange calculated from the dispersion relation is taken into account by the $f(s)$ term in the propagator of $T_{\rho}^{\mathrm{GS}}(s)$. Unitarity far from the pole mass is thus ensured. Explicitly,

$f(s)=\Gamma_{\rho}^{0} \frac{m_{\rho}^{2}}{q_{0}^{3}}\left[q^{2}\left[h(\sqrt{s})-h\left(m_{\rho}\right)\right]+\left.\left(m_{\rho}^{2}-s\right) q_{0}^{2} \frac{d h}{d s}\right|_{m_{\rho}}\right]$

and

$$
\begin{aligned}
h(s) & =\frac{2}{\pi} \frac{q}{\sqrt{s}} \ln \left(\frac{\sqrt{s}+2 q}{2 m_{\pi}}\right), \\
\left.\frac{d h}{d s}\right|_{m_{\rho}} & =h\left(m_{\rho}\right)\left[\frac{1}{8 q_{0}^{2}}-\frac{1}{2 m_{\rho}^{2}}\right]+\frac{1}{2 \pi m_{\rho}^{2}} .
\end{aligned}
$$

The constant parameter $D$ is given by

$$
D=\frac{3}{\pi} \frac{m_{\pi}^{2}}{q_{0}^{2}} \ln \left(\frac{m_{\rho}+2 q_{0}}{2 m_{\pi}}\right)+\frac{m_{\rho}}{2 \pi q_{0}}-\frac{m_{\pi}^{2} m_{\rho}}{\pi q_{0}^{3}} .
$$

The $\left(1+D \Gamma_{\rho}^{0} / m_{\rho}\right)$ factor in Eq. (2.25) will modify the relation in Eq. (2.15) into

$$
\begin{aligned}
& i \frac{\sqrt{\pi m_{R} \Gamma_{R}^{0}}}{\left(1+D \Gamma_{\rho}^{0} / m_{\rho}\right)} A^{\mathrm{GS}}\left(m_{\rho}, m_{23}\right) \\
& =\sum_{\lambda} \mathcal{M}_{\lambda}\left[B \rightarrow \rho\left(m_{R}\right) P_{3}\right] \frac{\mathcal{M}_{\lambda}\left[\rho\left(m_{R}\right) \rightarrow P_{1} P_{2}\right]}{\sqrt{m_{R} \Gamma_{R}^{0} / \pi}}
\end{aligned}
$$

instead. It can be easily seen that Eqs. (2.6), (2.7), and (2.20) all need to be corrected by the factor of $1 /(1+$ $\left.D \Gamma_{\rho}^{0} / m_{\rho}\right)^{2}$ accordingly. More explicitly, Eqs. (2.7) and (2.20) should be replaced by

$\eta_{\rho}^{\mathrm{GS}}=\frac{\pi m_{\rho} \Gamma_{\rho}}{\left(1+D \Gamma_{\rho}^{0} / m_{\rho}\right)^{2}} \frac{\int\left|A\left(m_{\rho}, m_{23}\right)\right|^{2} d m_{23}^{2}}{\int\left|A\left(m_{12}, m_{23}\right)\right|^{2} d m_{12}^{2} d m_{23}^{2}}$

and

$$
\eta_{\rho}^{\mathrm{GS}}=\frac{\pi m_{\rho} \Gamma_{\rho}^{0}}{\left(1+D \Gamma_{\rho}^{0} / m_{\rho}\right)^{2}} \frac{d \tilde{\Gamma}\left(m_{\rho}^{2}\right)}{d m_{12}^{2}}=\frac{\pi \Gamma_{\rho}^{0}}{2\left(1+D \Gamma_{\rho}^{0} / m_{\rho}\right)^{2}} \frac{d \tilde{\Gamma}\left(m_{\rho}\right)}{d m_{12}},
$$

respectively.

\section{E. Formula of $\eta_{R}$ in the case of the $\sigma / f_{0}(500)$ resonance}

As stressed in [11], the scalar resonance $\sigma / f_{0}(500)$ is very broad and cannot be described by the usual BreitWigner line shape. The partial wave amplitude does not resemble a Breit-Wigner shape with a clear peak and a simultaneous steep rise in the phase. The mass and width of the $\sigma$ resonance are identified from the associated pole position $\sqrt{s_{\sigma}}$ of the partial wave amplitude in the second Riemann sheet as $\sqrt{s_{\sigma}}=m_{\sigma}-i \Gamma_{\sigma} / 2$ [11]. Hence, we shall follow the LHCb Collaboration [10] to use a simple pole description

$T_{\sigma}(s)=\frac{1}{s-s_{\sigma}}=\frac{1}{s-m_{\sigma}^{2}+\Gamma_{\sigma}^{2}(s) / 4+i m_{\sigma} \Gamma_{\sigma}(s)}$,

with

$$
\Gamma_{\sigma}(s)=\Gamma_{\sigma}^{0}\left(\frac{q}{q_{0}}\right) \frac{m_{\sigma}}{\sqrt{s}}
$$

and $\Gamma_{\sigma}\left(m_{\sigma}^{2}\right)=\Gamma_{\sigma}^{0}$.

The factor of $1 /\left[\left(\Gamma_{\sigma}^{0}\right)^{2} / 4+i m_{\sigma} \Gamma_{\sigma}^{0}\right]=\left(i m_{\sigma} \Gamma_{\sigma}^{0}\right)^{-1}(1-$ $\left.i \Gamma_{\sigma}^{0} / 4 m_{\sigma}\right)^{-1}$ in Eq. (2.33) at the resonance will modify the relation in Eq. (2.15) into

$$
\begin{aligned}
& \left(1-i \frac{\Gamma_{\sigma}^{0}}{4 m_{\sigma}}\right) i \sqrt{\pi m_{R} \Gamma_{R}^{0}} A\left(m_{\sigma}, m_{23}\right) \\
& =\sum_{\lambda} \mathcal{M}_{\lambda}\left[B \rightarrow \sigma\left(m_{R}\right) P_{3}\right] \frac{\mathcal{M}_{\lambda}\left[\sigma\left(m_{R}\right) \rightarrow P_{1} P_{2}\right]}{\sqrt{m_{R} \Gamma_{R}^{0} / \pi}}
\end{aligned}
$$

instead. It can be easily seen that Eqs. (2.6), (2.7), and (2.20) all need to be corrected by the factor of $r_{\sigma} \equiv[1+$ $\left.\left(\Gamma_{\sigma}^{0} / 4 m_{\sigma}\right)^{2}\right]$ accordingly. More explicitly, Eqs. (2.7) and (2.20) should be replaced by

$$
\eta_{\sigma}=\pi r_{\sigma} m_{\sigma} \Gamma_{\sigma} \frac{\int\left|A\left(m_{\sigma}, m_{23}\right)\right|^{2} d m_{23}^{2}}{\int\left|A\left(m_{12}, m_{23}\right)\right|^{2} d m_{12}^{2} d m_{23}^{2}}
$$

and

$$
\eta_{\sigma}=\pi r_{\sigma} m_{\sigma} \Gamma_{\sigma}^{0} \frac{d \tilde{\Gamma}\left(m_{\sigma}^{2}\right)}{d m_{12}^{2}}=\frac{\pi r_{\sigma} \Gamma_{\sigma}^{0}}{2} \frac{d \tilde{\Gamma}\left(m_{\sigma}\right)}{d m_{12}},
$$

respectively.

\section{DIFFERENTIAL RATES AND $\eta_{R}^{\text {EXPP }}$ USING THE EXPERIMENTAL PARAMETRIZATION}

The following parametrization of the decay amplitude is widely used in the experimental studies of $B \rightarrow R P_{3} \rightarrow$ $P_{1} P_{2} P_{3}$ decays (see, e.g., [12]):

$$
\begin{aligned}
A\left(m_{12}, m_{23}\right)= & c F\left(m_{12}, m_{23}\right) \\
= & c R_{J}\left(m_{12}\right) \times X_{J}\left(p_{3}\right) \\
& \times X_{J}\left(p_{1}\right) \times T_{J}\left(m_{12}, m_{23}\right),
\end{aligned}
$$

where $R_{J}$ describes the line shape of the resonance introduced before in Eq. (2.10), $X_{J}$ is the BlattWeisskopf barrier form factor as defined in Eq. (2.12) with both $p_{1}$ and $p_{3}$ evaluated in the $R\left(m_{12}\right)$ rest frame, $T_{J}\left(m_{12}, m_{23}\right)$ is an angular distribution term given by [6] 


$$
\begin{aligned}
T_{0}\left(m_{12}, m_{23}\right)= & 1, \\
T_{1}\left(m_{12}, m_{23}\right)= & m_{23}^{2}-m_{13}^{2}+\frac{\left(m_{B}^{2}-m_{3}^{2}\right)\left(m_{1}^{2}-m_{2}^{2}\right)}{m_{12}^{2}}, \\
T_{2}\left(m_{12}, m_{23}\right)= & \left(m_{23}^{2}-m_{13}^{2}+\frac{\left(m_{B}^{2}-m_{3}^{2}\right)\left(m_{1}^{2}-m_{2}^{2}\right)}{m_{12}^{2}}\right)^{2}-\frac{1}{3}\left(m_{12}^{2}-2 m_{B}^{2}-2 m_{3}^{2}+\frac{\left(m_{B}^{2}-m_{3}^{2}\right)^{2}}{m_{12}^{2}}\right) \\
& \times\left(m_{12}^{2}-2 m_{1}^{2}-2 m_{2}^{2}+\frac{\left(m_{1}^{2}-m_{2}^{2}\right)^{2}}{m_{12}^{2}}\right),
\end{aligned}
$$

and $c$ is an unknown complex coefficient to be fitted to the data. Basically the information of weak decay amplitude is included in $c$. However, it is assumed to be a constant that has no dependence on energy or momentum of the decay products.

The quantities $\Gamma\left(B \rightarrow R P_{3}\right) \mathcal{B}\left(R \rightarrow P_{1} P_{2}\right)$ and $\eta_{R}^{\mathrm{EXPP}}$ can be obtained by using Eqs. (2.6) and (2.7) as

$$
\begin{aligned}
\Gamma\left(B \rightarrow R P_{3}\right) \mathcal{B}\left(R \rightarrow P_{1} P_{2}\right)= & \frac{|c|^{2}}{8 \pi^{2} m_{R} \Gamma_{R}} \frac{1}{32 m_{B}^{3}} \int_{\left(m_{23}^{2}\right)_{\min }\left(m_{R}\right)}^{\left(m_{22}^{2}\right)_{\max }\left(m_{R}\right)}\left(\left|X_{J}\left(p_{3}\right)\right|^{2}\left|X_{J}\left(p_{1}\right)\right|^{2}\right)_{m_{12} \rightarrow m_{R}} \\
& \times\left|T_{J}\left(m_{R}, m_{23}\right)\right|^{2} d m_{23}^{2}
\end{aligned}
$$

and

$$
\eta_{R}^{\mathrm{EXPP}}=\frac{\pi}{m_{R} \Gamma_{R}} \frac{\int_{\left(m_{23}^{3}\right) \min }^{\left(m_{\min }^{2}\right)_{\max }\left(m_{R}\right)}\left(\left|X_{J}\left(p_{3}\right)\right|^{2}\left|X_{J}\left(p_{1}\right)\right|^{2}\right)_{m_{12} \rightarrow m_{R}} \times\left|T_{J}\left(m_{R}, m_{23}\right)\right|^{2} d m_{23}^{2}}{\int\left|R_{J}\left(m_{12}\right) \times X_{J}\left(p_{3}\right) \times X_{J}\left(p_{1}\right) \times T_{J}\left(m_{12}, m_{23}\right)\right|^{2} d m_{12}^{2} d m_{23}^{2}} .
$$

Note that being a constant, the factor $c$ in $A\left(m_{12}, m_{23}\right)$ is canceled out between the numerator and the denominator in $\eta_{R}^{\text {EXPP }}$. One can readily verify that $\eta_{R}^{\text {EXPP }}$ approaches unity in the narrow width limit by virtue of Eq. (2.8).

We can express $\eta_{R}^{\text {EXPP }}$ in terms of the normalized differential rate,

$$
\eta_{R}^{\mathrm{EXPP}}=\pi m_{R} \Gamma_{R} \frac{d \tilde{\Gamma}\left(m_{R}^{2}\right)}{d m_{12}^{2}}=\frac{1}{2} \pi \Gamma_{R} \frac{d \tilde{\Gamma}\left(m_{R}\right)}{d m_{12}},
$$

with

$$
\frac{d \tilde{\Gamma}\left(m_{12}^{2}\right)}{d m_{12}^{2}}=\frac{\left|R_{J}\left(m_{12}\right)\right|^{2} \int\left|X_{J}\left(p_{3}\right) \times X_{J}\left(p_{1}\right) \times T_{J}\left(m_{R}, m_{23}\right)\right|^{2} d m_{23}^{2}}{\int\left|R_{J}\left(m_{12}\right) \times X_{J}\left(p_{3}\right) \times X_{J}\left(p_{1}\right) \times T_{J}\left(m_{12}, m_{23}\right)\right|^{2} d m_{12}^{2} d m_{23}^{2}} .
$$

In the case that $P_{2}$ and $P_{3}$ are identical particles, we shall use Eq. (2.18) to obtain $\eta_{R}^{\mathrm{EXPP}}$, giving

$$
\eta_{R}^{\mathrm{EXPP}}=\frac{2 \pi \int_{\left(m_{23}^{2}\right)_{\min }\left(m_{R}\right)}^{\left(m^{2}\right)_{\operatorname{man}}\left(m_{R}\right)}\left(\left|X_{J}\left(p_{3}\right)\right|^{2}\left|X_{J}\left(p_{1}\right)\right|^{2}\right)_{m_{12} \rightarrow m_{R}} \times\left|T_{J}\left(m_{R}, m_{23}\right)\right|^{2} d m_{23}^{2}}{m_{R} \Gamma_{R} \int\left|R_{J}\left(m_{12}\right) \times X_{J}\left(p_{3}\right) \times X_{J}\left(p_{1}\right) \times T_{J}\left(m_{12}, m_{23}\right)+(2 \leftrightarrow 3)\right|^{2} d m_{12}^{2} d m_{23}^{2}} .
$$

Note that when the Gounaris-Sakurai line shape is used in place of $R_{J}$, we should use Eqs. (2.31) and (2.32), instead of Eq. (3.5), while Eqs. (3.4) and (3.6) are still valid. For the case of the $\sigma$ resonance, we should use Eqs. (2.36) and (2.37).

In the case of narrow width, it is legitimate to use a complex constant $c$ to represent the weak dynamics. As noted previously, it is the shape of the entire normalized differential rate that matters in determining $\eta_{R}$. Hence, in the case of finite width, the momentum dependence in the weak amplitude will play some role.

There are some subtleties in the angular terms. Note that Eq. (3.2) was obtained with transversality conditions, $p_{12}^{\mu} \epsilon_{\mu}=0$ and $p_{12}^{\mu} p_{12}^{\nu} \epsilon_{\mu \nu}=0$, enforced for $J=1,2$ [6], 


$$
\begin{aligned}
T_{1}\left(m_{12}, m_{23}\right) & =\sum_{\lambda}\left(p_{B}+p_{3}\right)_{\mu} \epsilon^{* \mu}\left(p_{12}, \lambda\right) \epsilon^{\nu}\left(p_{12}, \lambda\right)\left(p_{1}-p_{2}\right)_{\nu} \\
& =-2 \vec{p}_{1} \cdot \vec{p}_{3}=-2 q\left|\vec{p}_{3}\right| \cos \theta, \\
T_{2}\left(m_{12}, m_{23}\right) & =\sum_{\lambda}\left(p_{B}+p_{3}\right)_{\mu}\left(p_{B}+p_{3}\right)_{\nu} \epsilon^{* \mu \nu}\left(p_{12}, \lambda\right) \epsilon^{\alpha \beta}\left(p_{12}, \lambda\right)\left(p_{1}-p_{2}\right)_{\alpha}\left(p_{1}-p_{2}\right)_{\beta} \\
& =\frac{4}{3}\left[3\left(\vec{p}_{1} \cdot \vec{p}_{3}\right)^{2}-\left(\left|\vec{p}_{1}\right|\left|\vec{p}_{3}\right|\right)^{2}\right]=\frac{4}{3} q^{2}\left|\vec{p}_{3}\right|^{2}\left(3 \cos ^{2} \theta-1\right),
\end{aligned}
$$

where $\epsilon^{\mu}$ and $\epsilon^{\mu \nu}$ are the polarization vector and tensor, respectively, and

$$
\begin{aligned}
q=\left|\vec{p}_{1}\right|=\left|\vec{p}_{2}\right| & =\frac{\sqrt{\left[m_{12}^{2}-\left(m_{1}+m_{2}\right)^{2}\right]\left[m_{12}^{2}-\left(m_{1}-m_{2}\right)^{2}\right]}}{2 m_{12}}, \\
\left|\vec{p}_{3}\right| & =\left(\frac{\left(m_{B}^{2}-m_{3}^{2}-m_{12}^{2}\right)^{2}}{4 m_{12}^{2}}-m_{3}^{2}\right)^{1 / 2},
\end{aligned}
$$

with $q=\left|\vec{p}_{1,2}\right|$ and $\left|\vec{p}_{3}\right|$ being the momenta of $P_{1,2}$ and $P_{3}$ in the $R\left(m_{12}\right)$ rest frame, respectively. ${ }^{4}$ Note that in Eq. (3.8), the factor contracted with $\left(p_{B}+p_{3}\right)$ comes from the $B \rightarrow R\left(m_{12}\right) P$ weak decay amplitude, while the one contracted with $\left(p_{1}-p_{2}\right)$ comes from the $R\left(m_{12}\right) \rightarrow$ $P_{1} P_{2}$ strong decay amplitude. To obtain the $\cos \theta$ dependence, it is useful to recall $\sum_{\lambda} \epsilon_{\mu}^{*}\left(p_{12}, \lambda\right) \epsilon_{\nu}\left(p_{12}, \lambda\right)=g_{\mu}^{i} g_{\nu}^{j} \delta_{i j}$ in the rest fame of $R\left(m_{12}\right)$.

Alternatively, using the standard expressions of vector and tensor propagators, which are contracted with the $B \rightarrow$ $R\left(m_{12}\right) P$ and the $R\left(m_{12}\right) \rightarrow P_{1} P_{2}$ parts, we expect the angular terms to take the following forms:

$$
\begin{aligned}
T_{1}^{\prime}\left(m_{12}, m_{23}\right)= & m_{23}^{2}-m_{13}^{2}+\frac{\left(m_{B}^{2}-m_{3}^{2}\right)\left(m_{1}^{2}-m_{2}^{2}\right)}{m_{R}^{2}}, \\
T_{2}^{\prime}\left(m_{12}, m_{23}\right)= & \left(m_{23}^{2}-m_{13}^{2}+\frac{\left(m_{B}^{2}-m_{3}^{2}\right)\left(m_{1}^{2}-m_{2}^{2}\right)}{m_{R}^{2}}\right)^{2} \\
& -\frac{1}{3}\left(m_{12}^{2}-2 m_{B}^{2}-2 m_{3}^{2}+\frac{\left(m_{B}^{2}-m_{3}^{2}\right)^{2}}{m_{R}^{2}}\right) \\
& \times\left(m_{12}^{2}-2 m_{1}^{2}-2 m_{2}^{2}+\frac{\left(m_{1}^{2}-m_{2}^{2}\right)^{2}}{m_{R}^{2}}\right) .
\end{aligned}
$$

The transversality condition, however, is not imposed on the above equations as the denominators become $m_{R}^{2}$ instead of $m_{12}^{2}$. In general, these $T_{J}^{\prime}$ cannot be expressed as Eq. (3.8) except on the mass shell of $p_{12}$, where these two angular terms coincide, i.e., $T_{J}^{\prime}\left(m_{R}, m_{23}\right)=$ $T_{J}\left(m_{R}, m_{23}\right)$. In the case of a vector resonance, except

\footnotetext{
${ }^{4}$ Note that $\left|\vec{p}_{3}\right|$ is related to $\tilde{p}_{c}$ through the relation $\tilde{p}_{c}=$ $\left(m_{12} / m_{B}\right)\left|\vec{p}_{3}\right|$, where $\tilde{p}_{c}$ is the c.m. momentum of $P_{3}$ or $R\left(m_{12}\right)$ in the $B$ rest frame. This relation can be easily verified using the conservation of momentum.
}

for modes with the intermediate resonance decaying to daughters of different masses, these two angular terms are identical throughout the entire phase space. We will also consider the case where the transversality condition is not imposed.

\section{ANALYSIS IN THE QCD FACTORIZATION APPROACH}

In this section, we will evaluate the decay amplitudes of $B \rightarrow R P_{3}$ and $B \rightarrow R P_{3} \rightarrow P_{1} P_{2} P_{3}$ within the framework of QCD factorization [13,14]. For the latter, its general amplitude has the expression

$$
\begin{aligned}
A\left(B \rightarrow R P_{3} \rightarrow P_{1} P_{2} P_{3}\right) \\
\equiv \\
=A\left(m_{12}, m_{23}\right) \\
=g^{R \rightarrow P_{1} P_{2}} F\left(s_{12}, m_{R}\right) \tilde{A}\left(B \rightarrow R\left(m_{12}\right) P_{3}\right) \\
\quad \times R_{J}\left(m_{12}\right) \mathcal{T}_{J}\left(m_{12}, m_{23}\right),
\end{aligned}
$$

where $g^{R \rightarrow P_{1} P_{2}}$ is the strong coupling constant associated with the strong decay $R\left(m_{12}\right) \rightarrow P_{1} P_{2}, F\left(s, m_{R}\right)$ is a form factor to be introduced later [see Eq. (4.20) below], $R_{J}$ is the resonance line shape, and $\mathcal{T}_{J}$ is the angular distribution function. In this work, we find

$\mathcal{T}_{0}=1, \quad \mathcal{T}_{1}=2 q \cos \theta, \quad \mathcal{T}_{2}=\frac{q^{2}}{\sqrt{6}}\left(1-3 \cos ^{2} \theta\right)$,

where $\theta$ is the angle between $\vec{p}_{1}$ and $\vec{p}_{3}$ measured in the rest frame of the resonance and $q$ is given before in Eq. (3.9). In Eq. (4.1), the weak decay amplitude $\tilde{A}\left(B \rightarrow R\left(m_{12}\right) P_{3}\right)$ will be reduced to the QCDF amplitude $A\left(B \rightarrow R\left(m_{R}\right) P_{3}\right)$ when $m_{12} \rightarrow m_{R}$. 
Taking the relativistic Breit-Wigner line shape Eq. (2.10), it follows from Eqs. (2.2) and (2.4) that

$$
\begin{aligned}
\frac{d \Gamma\left(m_{R}^{2}\right)}{d m_{12}^{2}}= & \frac{1}{(2 \pi)^{5}} \frac{1}{32 m_{R} m_{B}^{2}} \int\left|A\left(m_{R}, m_{23}\right)\right|^{2}\left|\vec{p}_{1}\right|\left|\vec{p}_{3}\right| d \Omega_{1} d \Omega_{3}, \\
= & \frac{1}{\pi m_{R} \Gamma_{R}^{2}}\left(\frac{1}{32 \pi^{2}} \int\left|A\left(B \rightarrow R\left(m_{R}\right) P_{3}\right)\right|^{2} \frac{\left|\vec{p}_{3}\right|}{m_{B}^{2}} d \Omega_{3}\right) \\
& \times\left(\frac{1}{32 \pi^{2}} \int\left|g^{R \rightarrow P_{1} P_{2}} \mathcal{T}_{J}\right|^{2} \frac{\left|\vec{p}_{1}\right|}{m_{R}^{2}} d \Omega_{1}\right), \\
= & \frac{1}{\pi m_{R} \Gamma_{R}} \Gamma\left(B \rightarrow R P_{3}\right) \mathcal{B}\left(R \rightarrow P_{1} P_{2}\right) .
\end{aligned}
$$

Indeed, it is straightforward to show that the partial rate of $R\left(m_{R}\right) \rightarrow P_{1} P_{2}$ given by

$$
\Gamma\left(R \rightarrow P_{1} P_{2}\right)=\frac{1}{32 \pi^{2}} \int\left|g^{R \rightarrow P_{1} P_{2}} \mathcal{T}_{J}\right|^{2} \frac{q_{0}}{m_{R}^{2}} d \Omega_{1}
$$

has the following expressions (see also Eq. (2.39) of [15]):

$$
\begin{aligned}
& \Gamma_{S \rightarrow P_{1} P_{2}}=\frac{q_{0}}{8 \pi m_{S}^{2}} g_{S \rightarrow P_{1} P_{2}}^{2}, \quad \Gamma_{V \rightarrow P_{1} P_{2}}=\frac{q_{0}^{3}}{6 \pi m_{V}^{2}} g_{V \rightarrow P_{1} P_{2}}^{2}, \\
& \Gamma_{T \rightarrow P_{1} P_{2}}=\frac{q_{0}^{5}}{60 \pi m_{T}^{2}} g_{T \rightarrow P_{1} P_{2}}^{2}
\end{aligned}
$$

for different types of resonances. Therefore, the decay rate of $B \rightarrow R\left(m_{R}\right) P_{3}$ can be related to the differential rate of $B \rightarrow$ $R P_{3} \rightarrow P_{1} P_{2} P_{3}$ at the resonance. This means that $\eta_{R}$ can be obtained from the normalized differential rate as shown in Eq. (2.7) or (2.20).

Most of the input parameters employed in this section such as decay constants, form factors, Cabibbo-KobayashiMaskawa matrix elements can be found in Appendix A of [15].

\section{A. Tensor resonances}

We begin with the tensor resonances and consider the three-body decay processes: $B^{-} \rightarrow f_{2}(1270) \pi^{-} \rightarrow \pi^{+} \pi^{-} \pi^{-}$ and $B^{-} \rightarrow \bar{K}_{2}^{* 0}(1430) \pi^{-} \rightarrow K^{-} \pi^{+} \pi^{-}$. Since the decay widths of $f_{2}(1270)$ and $K_{2}^{*}(1430)$ are around 187 and $109 \mathrm{MeV}$, respectively, it is naïvely expected that the deviation of $\eta_{f_{2}}$ from unity is larger than that of $\eta_{K_{2}^{*}}$ in both QCDF and EXPP schemes. We shall see below that this is not respected in the QCDF scheme and barely holds in the EXPP scheme.

$$
\text { 1. } f_{\mathbf{2}}(\mathbf{1 2 7 0})
$$

$B^{-} \rightarrow f_{2}(1270) \pi^{-}$decay in $Q C D F$.-

Consider the process $B^{-} \rightarrow f_{2}(1270) \pi^{-} \rightarrow \pi^{+} \pi^{-} \pi^{-}$. In QCDF, the amplitude of the quasi-two-body decay $B^{-} \rightarrow$ $f_{2}(1270) \pi^{-}$is given by [16]

$$
\begin{aligned}
A\left(B^{-} \rightarrow f_{2}(1270) \pi^{-}\right)= & \frac{G_{F}}{2} \sum_{p=u, c} \lambda_{p}^{(d)}\left\{\left[a_{1} \delta_{p u}+a_{4}^{p}+a_{10}^{p}-\left(a_{6}^{p}+a_{8}^{p}\right) r_{\chi}^{\pi}+\beta_{2}^{p} \delta_{p u}+\beta_{3}^{p}+\beta_{3, \mathrm{EW}}^{p}\right]_{f_{2} \pi} X^{\left(B f_{2}, \pi\right)}\right. \\
& +\left[a_{2} \delta_{p u}+2\left(a_{3}^{p}+a_{5}^{p}\right)+a_{4}^{p}+r_{\chi}^{f_{2}} a_{6}^{p}+\frac{1}{2}\left(a_{7}^{p}+a_{9}^{p}\right)-\frac{1}{2}\left(a_{10}^{p}+r_{\chi}^{f_{2}} a_{8}^{p}\right)\right. \\
& \left.+\beta_{2}^{p} \delta_{p u}+\beta_{3}^{p}+\beta_{3, \mathrm{EW}}^{p}\right]_{\pi f_{2}}^{\left.X^{\left(B \pi, f_{2}\right)}\right\},}
\end{aligned}
$$

where $\lambda_{p}^{(d)} \equiv V_{p b} V_{p d}^{*}$ and

$$
X^{\left(B f_{2}, \pi\right)}=2 f_{\pi} A_{0}^{B f_{2}}\left(m_{\pi}^{2}\right) \frac{m_{f_{2}}}{m_{B}} \epsilon^{* \mu \nu}(0) p_{B \mu} p_{B \nu}, \quad X^{\left(B \pi, f_{2}\right)}=2 f_{f_{2}} m_{B} p_{c} F_{1}^{B \pi}\left(m_{f_{2}}^{2}\right),
$$

with $p_{c}$ being the c.m. momentum of either $f_{2}$ or $\pi^{-}$in the $B$ rest frame. The chiral factors $r_{\chi}^{\pi}$ and $r_{\chi}^{f_{2}}$ in Eq. (4.6) are given by

$$
r_{\chi}^{\pi}(\mu)=\frac{2 m_{\pi}^{2}}{m_{b}(\mu)\left(m_{u}+m_{d}\right)(\mu)}, \quad r_{\chi}^{f_{2}}(\mu)=\frac{2 m_{f_{2}}}{m_{b}(\mu)} \frac{f_{f_{2}}^{\perp}(\mu)}{f_{f_{2}}}
$$

For the definition of the scale-dependent decay constants $f_{f_{2}}$ and $f_{f_{2}}^{\perp}$, see, for example, Ref. [16]. The coefficients $\beta_{i}^{p}$ describe weak annihilation contributions to the decay. The order of the arguments in the $a_{i}^{p}\left(M_{1} M_{2}\right)$ and $\beta_{i}^{p}\left(M_{1} M_{2}\right)$ coefficients is dictated by the subscript $M_{1} M_{2}$ given in Eq. (4.6).
In Eq. (4.7), $X^{\left(B f_{2}, \pi\right)}$ is factorizable and given by $\left\langle\pi\left|J^{\mu}\right| 0\right\rangle\left\langle f_{2}\left|J_{\mu}^{\prime}\right| B\right\rangle$, while $X^{\left(B \pi, f_{2}\right)}$ is a nonfactorizable amplitude as the factorizable one $\left\langle f_{2}\left|J^{\mu}\right| 0\right\rangle\left\langle\pi^{-}\left|J_{\mu}^{\prime}\right| B^{-}\right\rangle$ vanishes owing to the fact that the tensor meson cannot be produced through the $V-A$ current. Nevertheless, beyond the factorization approximation, contributions 
proportional to the decay constant $f_{f_{2}}$ can be produced from vertex, penguin, and spectator-scattering corrections [16]. Therefore, when the strong coupling $\alpha_{s}$ is turned off, the nonfactorizable contributions vanish accordingly.

The factorizable amplitude $X^{\left(B f_{2}, \pi\right)}$ can be further simplified by working in the $B$ rest frame and assuming that $f_{2}(\pi)$ moves along the $-z(z)$ axis [16]. In this case, $p_{B}^{\mu}=\left(m_{B}, 0,0,0\right)$ and $\epsilon^{* \mu \nu}(0)=\sqrt{2 / 3} \epsilon^{* \mu}(0) \epsilon^{* \nu}(0)$ with $\epsilon^{* \mu}(0)=\left(p_{c}, 0,0, E_{f_{2}}\right)^{\mu} / m_{f_{2}}$ and, consequently,

$$
X^{\left(B f_{2}, \pi\right)}=2 \sqrt{\frac{2}{3}} f_{\pi} \frac{m_{B}}{m_{f_{2}}} p_{c}^{2} A_{0}^{B f_{2}}\left(m_{\pi}^{2}\right) .
$$

Three-body decay $B^{-} \rightarrow f_{2}(1270) \pi^{-} \rightarrow \pi^{+} \pi^{-} \pi^{-}$.-

As shown in [15], the decay amplitude $\mathcal{A}_{f_{2}(1270)} \equiv$ $A\left(B^{-} \rightarrow \pi^{-} f_{2}(1270) \rightarrow \pi^{-}\left(p_{5}\right) \pi^{+}\left(p_{2}\right) \pi^{-}\left(p_{3}\right)\right)$ evaluated in the factorization approach ${ }^{5}$ arises from the matrix element $\left\langle\pi^{+}\left(p_{2}\right) \pi^{-}\left(p_{3}\right)|(\bar{u} b)| B^{-}\right\rangle^{f_{2}}\left\langle\pi^{-}\left(p_{1}\right)|(\bar{d} u)| 0\right\rangle$, where $\left(\bar{q}_{1} q_{2}\right) \equiv \bar{q}_{1} \gamma_{\mu}\left(1-\gamma_{5}\right) q_{2}$ and the superscript $f_{2}$ denotes the contribution from the $f_{2}$ resonance to the matrix element $\left\langle\pi^{+}\left(p_{2}\right) \pi^{-}\left(p_{3}\right)|(\bar{u} b)| B^{-}\right\rangle$. We shall use the relativistic Breit-Wigner line shape to describe the distribution of $f_{2}(1270)$,

$$
T_{f_{2}}^{\mathrm{BW}}(s)=\frac{1}{s-m_{f_{2}}^{2}+i m_{f_{2}} \Gamma_{f_{2}}(s)},
$$

with

$$
\Gamma_{f_{2}}(s)=\Gamma_{f_{2}}^{0}\left(\frac{q}{q_{0}}\right)^{5} \frac{m_{f_{2}}}{\sqrt{s}} \frac{X_{2}^{2}(q)}{X_{2}^{2}\left(q_{0}\right)},
$$

where the quantities $q, q_{0}, X_{2}$, and $\Gamma_{T}^{0}$ are already introduced before in Eq. (2.11). One advantage of using the energy-dependent decay width is that $\Gamma_{f_{2}}(s)$ will vanish when $s$ goes below the $2 \pi$ threshold.

Consequently,

$$
\begin{aligned}
& \left\langle\pi^{+}\left(p_{2}\right) \pi^{-}\left(p_{3}\right)|(\bar{u} b)| B^{-}\right\rangle^{f_{2}}\left\langle\pi^{-}\left(p_{1}\right)|(\bar{d} u)| 0\right\rangle \\
& =\left\langle\pi^{+}\left(p_{2}\right) \pi^{-}\left(p_{3}\right) \mid f_{2}\right\rangle T_{f_{2}}^{\mathrm{BW}}\left(s_{23}\right)\left\langle f_{2}|(\bar{u} b)| B^{-}\right\rangle\left\langle\pi^{-}\left(p_{1}\right)|(\bar{d} u)| 0\right\rangle \\
& =\sum_{\lambda} \varepsilon^{* \mu \nu}(\lambda) p_{2 \mu} p_{3 \nu} g^{f_{2} \rightarrow \pi^{+} \pi^{-}} T_{f_{2}}^{\mathrm{BW}}\left(s_{23}\right) \frac{2 m_{f_{2}}}{m_{B}} f_{\pi} A_{0}^{B f_{2}}\left(m_{\pi}^{2}\right) \varepsilon_{\alpha \beta}(\lambda) p_{B}^{\alpha} p_{1}^{\beta} \\
& =\frac{2 m_{f_{2}}}{m_{B}} g^{f_{2} \rightarrow \pi^{+} \pi^{-}} f_{\pi} A_{0}^{B f_{2}}\left(m_{\pi}^{2}\right) T_{f_{2}}^{\mathrm{BW}}\left(s_{23}\right)\left[\frac{1}{3}\left(\left|\vec{p}_{1} \| \vec{p}_{2}\right|\right)^{2}-\left(\vec{p}_{1} \cdot \vec{p}_{2}\right)^{2}\right],
\end{aligned}
$$

where we have followed [20] for the definition of the $B \rightarrow T$ transition form factors $^{6}$ and employed the relation $[6,22]$

$$
\sum_{\lambda} \varepsilon^{* \mu \nu}(\lambda) \varepsilon_{\alpha \beta}(\lambda) p_{2 \mu} p_{3 \nu} p_{B}^{\alpha} p_{1}^{\beta}=\frac{1}{3}\left(\left|\vec{p}_{1}\right|\left|\vec{p}_{2}\right|\right)^{2}-\left(\vec{p}_{1} \cdot \vec{p}_{2}\right)^{2}
$$

with

$$
\left|\vec{p}_{1}\right|=\left(\frac{\left(m_{B}^{2}-m_{\pi}^{2}-s_{23}\right)^{2}}{4 s_{23}}-m_{\pi}^{2}\right)^{1 / 2}, \quad\left|\vec{p}_{2}\right|=\left|\vec{p}_{3}\right|=q=\frac{1}{2} \sqrt{s_{23}-4 m_{\pi}^{2}}
$$

Hence, factorization leads to

$$
\begin{aligned}
\mathcal{A}_{f_{2}(1270)}= & \frac{1}{\sqrt{2}} \frac{G_{F}}{\sqrt{2}} \sum_{p=u, c} \lambda_{p}^{(d)}\left[a_{1} \delta_{p u}+a_{4}^{p}+a_{10}^{p}-\left(a_{6}^{p}+a_{8}^{p}\right) r_{\chi}^{\pi}\right] \\
& \times \frac{2 m_{f_{2}}}{m_{B}} g^{f_{2} \rightarrow \pi^{+} \pi^{-}} f_{\pi^{\prime}} A_{0}^{B f_{2}}\left(m_{\pi}^{2}\right) T_{f_{2}}^{\mathrm{BW}}\left(s_{23}\right) \frac{1}{3}\left|\vec{p}_{1}\right|^{2}\left|\vec{p}_{2}\right|^{2}\left(1-3 \cos ^{2} \theta\right)+\left(s_{23} \leftrightarrow s_{12}\right),
\end{aligned}
$$

where the identical particle effect due to the two identical $\pi^{-}$has been taken into account. Comparing with Eq. (4.6), we see that the nonfactorizable contribution characterized by $X^{\left(B \pi, f_{2}\right)}$ and the weak annihilation described by $\beta^{p}$ terms are absent in the naïve factorization approach. We shall use the QCDF expression for $B^{-} \rightarrow f_{2}(1270) \pi^{-}$and write

\footnotetext{
${ }^{5}$ The study of charmless three-body $B$ decays in the factorization approach can be found in [15,17-19] and references therein.

${ }^{6}$ The $B \rightarrow T$ transition form factors defined in [16,20] differ by a factor of $i$. We shall use the former as they are consistent with the normalization of $B \rightarrow S$ transition given in [21].
} 


$$
\mathcal{A}_{f_{2}(1270)}=g^{f_{2} \rightarrow \pi^{+} \pi^{-}} T_{f_{2}}^{\mathrm{BW}}\left(s_{23}\right) \frac{q^{2}}{\sqrt{6}}\left(1-3 \cos ^{2} \theta\right) \tilde{A}\left(B^{-} \rightarrow f_{2}\left(m_{23}\right) \pi^{-}\right)+\left(s_{23} \leftrightarrow s_{12}\right),
$$

with

$$
\begin{aligned}
\tilde{A}\left(B^{-} \rightarrow f_{2}\left(m_{23}\right) \pi^{-}\right)= & \frac{G_{F}}{2} \sum_{p=u, c} \lambda_{p}^{(d)} \frac{m_{f_{2}}^{2}}{s_{23}}\left\{\left[a_{1} \delta_{p u}+a_{4}^{p}+\cdots+\beta_{3, \mathrm{EW}}^{p}\right]_{f_{2} \pi} \tilde{X}^{\left(B f_{2}, \pi\right)}\right. \\
& \left.+\left[a_{2} \delta_{p u}+2\left(a_{3}^{p}+a_{5}^{p}\right)+\cdots+\beta_{3, \mathrm{EW}}^{p}\right]_{\pi f_{2}} \tilde{X}^{\left(B \pi, f_{2}\right)}\right\}
\end{aligned}
$$

where

$$
\tilde{X}^{\left(B f_{2}, \pi\right)}=2 \sqrt{\frac{2}{3}} f_{\pi} \frac{m_{B}}{m_{f_{2}}} \tilde{p}_{c}^{2} A_{0}^{B f_{2}}\left(m_{\pi}^{2}\right), \quad \tilde{X}^{\left(B \pi, f_{2}\right)}=2 f_{f_{2}} m_{B} \tilde{p}_{c} F_{1}^{B \pi}\left(s_{23}\right),
$$

and

$$
\tilde{p}_{c}=\left(\frac{\left(m_{B}^{2}-m_{\pi}^{2}-s_{23}\right)^{2}}{4 m_{B}^{2}}-m_{\pi}^{2}\right)^{1 / 2}
$$

It is easily seen that $\tilde{A}\left(B^{-} \rightarrow f_{2}\left(m_{23}\right) \pi^{-}\right)$is reduced to the QCDF amplitude $A\left(B^{-} \rightarrow f_{2} \pi^{-}\right)$given in Eq. (4.6) when $m_{23} \rightarrow m_{f_{2}}$.

Before proceeding, we would like to address an issue. The strong coupling constant $\left|g^{f_{2}(1270) \rightarrow \pi^{+} \pi^{-}}\right|=$ $18.56 \mathrm{GeV}^{-1}$ extracted from the measured $f_{2}(1270)$ width [see Eq. (4.27) below] is for the physical $f_{2}(1270)$. When $f_{2}$ is off the mass shell, especially when $s_{23}$ is approaching the upper bound of $\left(m_{B}-m_{\pi}\right)^{2}$, it is necessary to account

for the off-shell effect. For this purpose, we shall follow [23] to introduce a form factor $F\left(s, m_{R}\right)$ parametrized as ${ }^{7}$

$$
F\left(s, m_{R}\right)=\left(\frac{\Lambda^{2}+m_{R}^{2}}{\Lambda^{2}+s}\right)^{n},
$$

with the cutoff $\Lambda$ not far from the resonance,

$$
\Lambda=m_{R}+\beta \Lambda_{\mathrm{QCD}}
$$

where the parameter $\beta$ is expected to be of order unity. We shall use $n=1, \Lambda_{\mathrm{QCD}}=250 \mathrm{MeV}$, and $\beta=1.0 \pm 0.2$ in subsequent calculations.

Finally, the decay rate is given by

$$
\begin{aligned}
\Gamma\left(B^{-} \rightarrow f_{2} \pi^{-} \rightarrow \pi^{+} \pi^{-} \pi^{-}\right)= & \frac{1}{2} \frac{1}{(2 \pi)^{3} m_{B}^{3}} \int_{\left(m_{\pi}+m_{\pi}\right)^{2}}^{\left(m_{B}-m_{\pi}\right)^{2}} d s_{23} \int_{\left(s_{12}\right)_{\min }}^{\left(s_{12}\right)_{\max }} d s_{12}\left|\mathcal{A}_{f_{2}}\right|^{2} \\
= & \frac{1}{2} \frac{1}{(2 \pi)^{3} 32 m_{B}^{3}} \int_{\left(m_{\pi}+m_{\pi}\right)^{2}}^{\left(m_{B}-m_{\pi}\right)^{2}} d s_{23} \int_{\left(s_{12}\right)_{\min }}^{\left(s_{12}\right)_{\max }} d s_{12}\left\{\frac{\left|g^{f_{2} \rightarrow \pi^{+} \pi^{-}}\right|^{2} F\left(s_{23}, m_{f_{2}}\right)^{2}}{\left(s_{23}-m_{f_{2}}^{2}\right)^{2}+m_{f_{2}}^{2} \Gamma_{f_{2}}^{2}\left(s_{23}\right)}\right. \\
& \left.\times \frac{q^{4}}{6}\left(1-3 \cos ^{2} \theta\right)^{2}\left|\tilde{A}\left(B^{-} \rightarrow f_{2} \pi^{-}\right)\right|^{2}+\left(s_{23} \leftrightarrow s_{12}\right)+\text { interference terms }\right\},
\end{aligned}
$$

where the factor of $1 / 2$ accounts for the identical-particle effect. Note that $\cos \theta$ can be expressed as in terms of $s_{12}$ and $s_{23}$,

$$
\cos \theta=a\left(s_{23}\right) s_{12}+b\left(s_{23}\right)
$$

with [24]

$$
\begin{gathered}
a(s)=\frac{1}{\left(s-4 m_{\pi}^{2}\right)^{1 / 2}\left(\frac{\left(m_{B}^{2}-m_{\pi}^{2}-s\right)^{2}}{4 s}-m_{\pi}^{2}\right)^{1 / 2}}, \\
b(s)=-\frac{m_{B}^{2}+3 m_{\pi}^{2}-s}{2\left(s-4 m_{\pi}^{2}\right)^{1 / 2}\left(\frac{\left(m_{B}^{2}-m_{\pi}^{2}-s\right)^{2}}{4 s}-m_{\pi}^{2}\right)^{1 / 2}} .
\end{gathered}
$$

\footnotetext{
${ }^{7}$ Note that the form factor $F(t, m)$ used in [23] is for the $t$-channel off-shell effect.
} 
TABLE I. Numerical values of the flavor operators $a_{i}^{p}\left(M_{1} M_{2}\right)$ for $M_{1} M_{2}=f_{2}(1270) \pi$ and $\pi f_{2}(1270)$ at the scale $\mu=\bar{m}_{b}\left(\bar{m}_{b}\right)=4.18 \mathrm{GeV}$.

\begin{tabular}{lcclcc}
\hline \hline$a_{i}^{p}$ & $f_{2} \pi$ & $\pi f_{2}$ & $a_{i}^{p}$ & $f_{2} \pi$ & $\pi f_{2}$ \\
\hline$a_{1}$ & $1.011+0.014 i$ & $-0.035+0.014 i$ & $a_{6}^{c}$ & $-0.053-0.005 i$ & $(6.3+1.6 i) 10^{-3}$ \\
$a_{2}$ & $0.123-0.080 i$ & $0.133-0.078 i$ & $a_{7}$ & $(-0.2+3.4 i) 10^{-5}$ & $(9.5-3.4 i) 10^{-5}$ \\
$a_{3}$ & $0.0014+0.0027 i$ & $-0.006+0.003 i$ & $a_{8}^{u}$ & $(3.6-1.0 i) 10^{-4}$ & $(-2.1+0.1 i) 10^{-5}$ \\
$a_{4}^{u}$ & $-0.027-0.014 i$ & $0.0064-0.0016 i$ & $a_{8}^{c}$ & $(3.4-0.5 i) 10^{-4}$ & $(3.3+1.0 i) 10^{-5}$ \\
$a_{4}^{c}$ & $-0.032-0.006 i$ & $0.0091+0.0064 i$ & $a_{9}$ & $(-9.1-0.1 i) 10^{-3}$ & $(3.0-1.2 i) 10^{-4}$ \\
$a_{5}$ & $0.0009-0.0031 i$ & $-0.008+0.003 i$ & $a_{10}^{u}$ & $(-8.2+6.2 i) 10^{-4}$ & $(-9.6+7.0 i) 10^{-4}$ \\
$a_{6}^{u}$ & $-0.050-0.014 i$ & $-(3.52+0.02 i) 10^{-3}$ & $a_{10}^{c}$ & $(-8.5+6.7 i) 10^{-4}$ & $(-9.4+7.5 i) 10^{-4}$ \\
\hline \hline
\end{tabular}

It follows that $\left(s_{12}\right)_{\min }=-(1+b) / a$ and $\left(s_{12}\right)_{\max }=$ $(1-b) / a$. It is straightforward to show that

$\int_{\left(s_{12}\right)_{\min }}^{\left(s_{12}\right)_{\max }} d s_{12}\left(1-3 \cos ^{2} \theta\right)^{2}=\frac{8}{5 a}=\frac{16}{5} \frac{m_{B}}{\sqrt{s_{23}}} q \tilde{p}_{c}$

In the narrow width limit, we have

$$
\frac{m_{f_{2}} \Gamma_{f_{2}}(s)}{\left(s-m_{f_{2}}^{2}\right)^{2}+m_{f_{2}}^{2} \Gamma_{f_{2}}^{2}(s)} \stackrel{\Gamma_{f_{2}} \rightarrow 0}{\longrightarrow} \pi \delta\left(s-m_{f_{2}}^{2}\right)
$$

Under the NWA, $\left|g^{f_{2} \rightarrow \pi^{+} \pi^{-}}\right|^{2} / \Gamma_{f_{2}}$ is finite as it is proportional to the branching fraction $\mathcal{B}\left(f_{2} \rightarrow \pi^{+} \pi^{-}\right)$. Due to the Dirac $\delta$-function in the above equation, we have $s_{23} \rightarrow m_{f_{2}}^{2}$ in the zero width limit. As a result, $\tilde{p}_{c} \rightarrow p_{c}, q \rightarrow q_{0}$, $\tilde{X}^{\left(B f_{2}, \pi\right)} \rightarrow X^{\left(B f_{2}, \pi\right)}, \quad \tilde{X}^{\left(B \pi, f_{2}\right)} \rightarrow X^{\left(B \pi, f_{2}\right)}, \quad$ and $\tilde{A}\left(B^{-} \rightarrow\right.$ $\left.f_{2} \pi^{-}\right) \rightarrow A\left(B^{-} \rightarrow f_{2} \pi^{-}\right)$. Likewise, the second term in Eq. (4.22) with the replacement $s_{23} \leftrightarrow s_{12}$ has a similar expression. However, the interference term vanishes in the NWA due to different $\delta$-functions. Using

$$
\Gamma_{f_{2} \rightarrow \pi^{+} \pi^{-}}=\frac{q_{0}^{5}}{60 \pi m_{f_{2}}^{2}}\left|g^{f_{2} \rightarrow \pi^{+} \pi^{-}}\right|^{2}, \quad \Gamma_{B^{-} \rightarrow f_{2} \pi^{-}}=\frac{p_{c}}{8 \pi m_{B}^{2}}\left|A\left(B^{-} \rightarrow f_{2} \pi^{-}\right)\right|^{2},
$$

we are led to the desired factorization relation,

$$
\Gamma\left(B^{-} \rightarrow f_{2} \pi^{-} \rightarrow \pi^{+} \pi^{-} \pi^{-}\right) \stackrel{\Gamma_{f_{2}} \rightarrow 0}{\longrightarrow} \Gamma\left(B^{-} \rightarrow f_{2} \pi^{-}\right) \mathcal{B}\left(f_{2} \rightarrow \pi^{+} \pi^{-}\right) .
$$

Numerical results.-

To compute $B^{-} \rightarrow f_{2} \pi^{-}$and the three-body decay $B^{-} \rightarrow f_{2} \pi^{-} \rightarrow \pi^{+} \pi^{-} \pi^{-}$, we need to know the values of the flavor operators $a_{i}^{p}\left(M_{1}, M_{2}\right)$. In the QCDF approach, the flavor operators have the expression [13,14]

$$
a_{i}^{p}\left(M_{1}, M_{2}\right)=\left(c_{i}+\frac{c_{i \pm 1}}{N_{c}}\right) N_{i}\left(M_{2}\right)+\frac{c_{i \pm 1}}{N_{c}} \frac{C_{F} \alpha_{s}}{4 \pi}\left[V_{i}\left(M_{2}\right)+\frac{4 \pi^{2}}{N_{c}} H_{i}\left(M_{1} M_{2}\right)\right]+P_{i}^{p}\left(M_{2}\right),
$$

where $i=1, \ldots, 10$, the upper (lower) sign is for odd (even) $i, c_{i}$ are the Wilson coefficients, $C_{F}=\left(N_{c}^{2}-1\right) /\left(2 N_{c}\right)$ with $N_{c}=3, M_{2}$ is the emitted meson, and $M_{1}$ shares the same spectator quark with the $B$ meson. The detailed expressions for the vertex corrections $V_{i}\left(M_{2}\right)$, hard spectator interactions $H_{i}\left(M_{1} M_{2}\right)$, and penguin contractions $P_{i}^{p}\left(M_{2}\right)$ for $M_{1} M_{2}=T P$ and $P T$ can be found in [16]. Note that the parameters $N_{i}(M)$ in Eq. (4.29) vanish if $M$ is a tensor meson; otherwise, it is equal to one. Therefore, the coefficient $a_{2}\left(\pi f_{2}\right)$ appearing in Eq. (4.6) vanishes when the strong coupling $\alpha_{s}$ is turned off. We see from Table I that $a_{i}^{p}\left(f_{2} P\right)$ and $a_{i}^{p}\left(P f_{2}\right)$ can be quite different.

It is known that power corrections in QCDF always involve troublesome endpoint divergences. For example, the annihilation amplitude has endpoint divergences even at twist-2 level, and the hard spectator scattering diagram at twist-3 order is power suppressed and possesses soft and collinear divergences arising from the soft spectator quark. Since the treatment of endpoint divergences is model dependent, we shall follow [13] to model the endpoint divergence $X \equiv \int_{0}^{1} d x / \bar{x}$ in the annihilation and hard spectator scattering diagrams as

$$
\begin{aligned}
& X_{A}=\ln \left(\frac{m_{B}}{\Lambda_{h}}\right)\left(1+\rho_{A} e^{i \phi_{A}}\right), \\
& X_{H}=\ln \left(\frac{m_{B}}{\Lambda_{h}}\right)\left(1+\rho_{H} e^{i \phi_{H}}\right),
\end{aligned}
$$


with $\Lambda_{h}$ being a typical hadronic scale of $0.5 \mathrm{GeV}$. In this work, we use

$$
\rho_{A}^{T P}=\rho_{A}^{P T}=0.7, \quad \phi_{A}^{T P}=\phi_{A}^{P T}=-30^{\circ},
$$

leading to

$$
\begin{aligned}
& \beta_{2}^{p}\left(f_{2} \pi\right)=0.023-0.010 i, \quad\left(\beta_{3}^{p}+\beta_{3, \mathrm{EW}}^{p}\right)\left(f_{2} \pi\right)=-0.047+0.053 i, \\
& \beta_{2}^{p}\left(\pi f_{2}\right)=-0.033+0.018 i, \quad\left(\beta_{3}^{p}+\beta_{3, \mathrm{EW}}^{p}\right)\left(\pi f_{2}\right)=-0.050+0.047 i
\end{aligned}
$$

for both $p=u$ and $c$.

Following [16], we obtain the branching fraction and $C P$ asymmetry for $B^{-} \rightarrow f_{2}(1270) \pi^{-}$as

$$
\begin{aligned}
\mathcal{B}\left(B^{-} \rightarrow f_{2}(1270) \pi^{-}\right)_{\mathrm{QCDF}} & =\left(2.65_{-1.22}^{+1.29}\right) \times 10^{-6}, \\
A_{C P}\left(B^{-}\right. & \left.\rightarrow f_{2}(1270) \pi^{-}\right)_{\mathrm{QCDF}}=\left(46.7_{-62.5}^{+32.6}\right) \%,
\end{aligned}
$$

where the decay constants $f_{f_{2}}=102 \pm 6 \mathrm{MeV}$ and $f_{f_{2}}^{\perp}=$ $117 \pm 25 \mathrm{MeV}$ both at $\mu=1 \mathrm{GeV}$ [25], the form factors $A_{0}^{B f_{2}(1270)}(0)=0.13 \pm 0.02$, derived from large energy effective theory (see Table II of [16]), and

$$
F_{1}^{B \pi}\left(q^{2}\right)=\frac{0.26 \pm 0.03}{1-\frac{q^{2}}{m_{B^{*}}^{2}}}\left(1+\frac{0.64 \frac{q^{2}}{m_{B^{*}}^{2}}}{1-0.40 \frac{q^{2}}{m_{B}^{2}}}\right)
$$

have been used. The theoretical errors correspond to the uncertainties due to the variation of Gegenbauer moments, decay constants, quark masses, form factors, the $\lambda_{B}$ parameter for the $B$ meson wave function, and the powercorrection parameters $\rho_{A, H}, \phi_{A, H}$ (see [16] for details), all added in quadrature. In the narrow width limit, we find the central values

$$
\begin{aligned}
\mathcal{B}\left(B^{-} \rightarrow f_{2}(1270) \pi^{-} \rightarrow \pi^{+} \pi^{-} \pi^{-}\right)_{\Gamma_{f_{2}} \rightarrow 0} & =1.485 \times 10^{-6}, \\
A_{C P}\left(B^{-} \rightarrow f_{2}(1270) \pi^{-} \rightarrow \pi^{+} \pi^{-} \pi^{-}\right)_{\Gamma_{f_{2}} \rightarrow 0} & =46.23 \% .
\end{aligned}
$$

Since $\mathcal{B}\left(f_{2}(1270) \rightarrow \pi^{+} \pi^{-}\right)=\left(0.842_{-0.009}^{+0.029}\right) \times \frac{2}{3}$, it is easily seen that the factorization relation Eq. (4.28) is indeed numerically valid in the narrow width limit.

For the finite-width $\Gamma_{f_{2}}^{0}=186.7_{-2.5}^{+2.2} \mathrm{MeV}$ [3], we find

$$
\begin{array}{rlrl}
\mathcal{B}\left(B^{-} \rightarrow f_{2}(1270) \pi^{-}\right. & \left.\rightarrow \pi^{+} \pi^{-} \pi^{-}\right)=\left(1.48_{-0.37}^{+0.42}\right) \times 10^{-6} \quad\left[\left(1.52_{-0.38}^{+0.43}\right) \times 10^{-6}\right], \\
A_{C P}\left(B^{-} \rightarrow f_{2}(1270) \pi^{-} \rightarrow \pi^{+} \pi^{-} \pi^{-}\right) & =\left(44.56_{-0.39}^{+0.41}\right) \% & {\left[\left(47.20_{-0.43}^{+0.45}\right) \%\right],}
\end{array}
$$

where the values in square parentheses are obtained with the form factor $F\left(s, m_{f_{2}}\right)$ being set as unity. They are in agreement with the recent $\mathrm{LHCb}$ measurements $[9,10]$,

$$
\begin{aligned}
\mathcal{B}\left(B^{-} \rightarrow f_{2}(1270) \pi^{-} \rightarrow \pi^{+} \pi^{-} \pi^{-}\right)_{\mathrm{LHCb}} & =(1.37 \pm 0.26) \times 10^{-6}, \\
A_{C P}\left(B^{-}\right. & \left.\rightarrow f_{2}(1270) \pi^{-} \rightarrow \pi^{+} \pi^{-} \pi^{-}\right)_{\mathrm{LHCb}}=(46.8 \pm 7.7) \%,
\end{aligned}
$$

and consistent with the earlier $B A B A R$ measurements [8],

$$
\begin{aligned}
\mathcal{B}\left(B^{-}\right. & \left.\rightarrow f_{2}(1270) \pi^{-} \rightarrow \pi^{+} \pi^{-} \pi^{-}\right)_{B A B A R}=\left(0.9 \pm 0.2_{-0.1}^{+0.3}\right) \times 10^{-6}, \\
A_{C P}\left(B^{-}\right. & \left.\rightarrow f_{2}(1270) \pi^{-} \rightarrow \pi^{+} \pi^{-} \pi^{-}\right)_{B A B A R}=\left(41 \pm 25_{-15}^{+18}\right) \% .
\end{aligned}
$$

Notice that a large $C P$ asymmetry in the $f_{2}(1270)$ component was firmly established by the LHCb Collaboration.

We are now in the position to compute the parameter $\eta_{f_{2}(1270)}$ defined in Eq. (1.3),

$$
\eta_{f_{2}}=\frac{\Gamma\left(B^{-} \rightarrow f_{2}(1270) \pi^{-}\right) \mathcal{B}\left(f_{2}(1270) \rightarrow \pi^{+} \pi^{-}\right)}{\Gamma\left(B^{-} \rightarrow f_{2}(1270) \pi^{-} \rightarrow \pi^{+} \pi^{-} \pi^{-}\right)} .
$$

From Eqs. (4.33) and (4.36), we find

$$
\eta_{f_{2}(1270)}^{\mathrm{QCDF}}=1.003_{-0.002}^{+0.001} \quad(0.9743 \pm 0.0003) .
$$




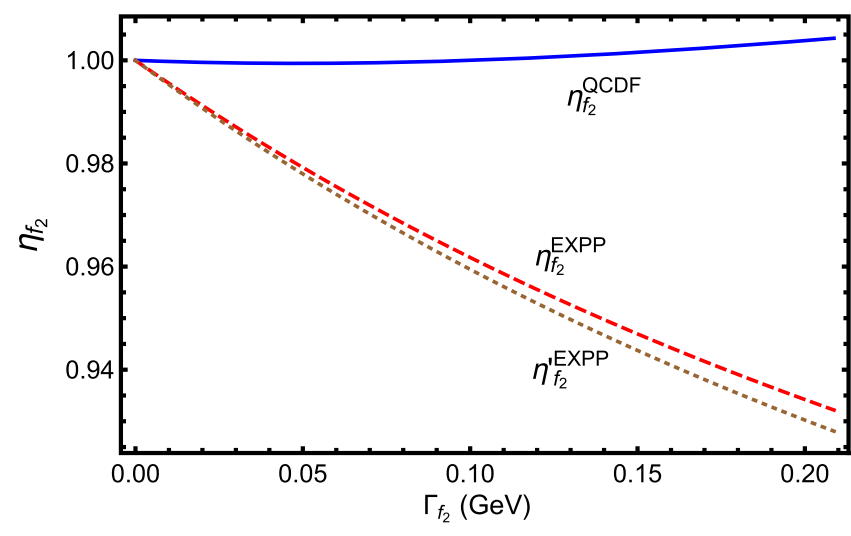

FIG. 1. The parameter $\eta_{f_{2}}$ as a function of the $f_{2}(1270)$ width, where the solid curve is derived from the QCDF calculation and the dashed (dotted) curve from the EXPP with (without) the transversality condition imposed.

Since the theoretical uncertainties in the numerator and denominator essentially cancel out, the errors on $\eta_{f_{2}}$ mainly arise from the uncertainties in $\beta$ and the $f_{2}$ width. As discussed in Sec. II, $\eta_{R}$ can be expressed in terms of the normalized differential rate. In general, the calculation done in this way is simpler. From Eqs. (2.18) and (4.16), we obtain the same result for $\eta_{f_{2}}^{\mathrm{QCDF}}$. The dependence of the parameter $\eta_{f_{2}}$ on the width $\Gamma_{f_{2}}$ is plotted as the solid blue curve in Fig. 1. It is somewhat surprising that the deviation of $\eta_{f_{2}}^{\mathrm{QCDF}}$ from unity is very tiny, even though $\Gamma_{f_{2}} / m_{f_{2}}$ is about 0.146 .

The parameter $\eta_{f_{2}}^{\text {EXPP }}$ is calculated using Eq. (2.18) together with the experimental parametrization, Eq. (3.1) for $A\left(m_{12}, m_{23}\right)$. Its dependence on the $f_{2}(1270)$ width is depicted by the dashed red curve in Fig. 1. At the resonance, we obtain

$$
\eta_{f_{2}(1270)}^{\mathrm{EXPP}}=0.937_{-0.005}^{+0.006}
$$

We see that with the physical width $\Gamma_{f_{2}}^{0}=186.7_{-2.5}^{+2.2} \mathrm{MeV}$, the results in the QCDF and EXPP schemes differ by about $7 \%$.

\section{2. $K_{2}^{*}(1430)$}

We next turn to the $B^{-} \rightarrow \bar{K}_{2}^{* 0}(1430) \pi^{-} \rightarrow K^{-} \pi^{+} \pi^{-}$ decay. The QCDF amplitude of the quasi-two-body $B^{-} \rightarrow$ $\bar{K}_{2}^{* 0}(1430) \pi^{-}$decay is given by [16]

$$
A\left(B^{-} \rightarrow \bar{K}_{2}^{* 0} \pi^{-}\right)=\frac{G_{F}}{\sqrt{2}} \sum_{p=u, c} \lambda_{p}^{(s)}\left[a_{4}^{p}+r_{\chi}^{K_{2}^{*}} a_{6}^{p}-\frac{1}{2}\left(a_{10}^{p}+r_{\chi}^{K_{2}^{*}} a_{8}\right)+\beta_{2}^{p} \delta_{p u}+\beta_{3}^{p}+\beta_{3, \mathrm{EW}}^{p}\right]_{\pi K_{2}^{*}} X^{\left(B \pi, \bar{K}_{2}^{*}\right)},
$$

with $\lambda_{p}^{(s)} \equiv V_{p b} V_{p s}^{*}$ and

$$
X^{\left(B \pi, K_{2}^{*}\right)}=2 f_{K_{2}^{*}} m_{B} p_{c} F_{1}^{B \pi}\left(m_{K_{2}^{*}}^{2}\right) .
$$

Note that this decay proceeds only through nonfactorizable diagrams.

Analogous to the $f_{2}(1270)$ resonance, the decay amplitude $\mathcal{A}_{K_{2}^{*}(1430)} \equiv A\left(B^{-} \rightarrow \bar{K}_{2}^{* 0}(1430) \pi^{-} \rightarrow K^{-}\left(p_{1}\right) \pi^{+}\left(p_{2}\right) \pi^{-}\left(p_{3}\right)\right.$ reads [see the second term of Eq. (4.16)]

$$
\mathcal{A}_{K_{2}^{*}(1430)}=g^{\bar{K}_{2}^{* 0} \rightarrow K^{-} \pi^{+}} F\left(s_{12}, m_{K_{2}^{*}}\right) T_{K_{2}^{*}}^{\mathrm{BW}}\left(s_{12}\right) \frac{q^{2}}{\sqrt{6}}\left(1-3 \cos ^{2} \theta\right) \tilde{A}\left(B^{-} \rightarrow \bar{K}_{2}^{* 0} \pi^{-}\right),
$$

with

$$
q=\frac{\sqrt{\left[s_{12}-\left(m_{K}+m_{\pi}\right)^{2}\right]\left[s_{12}-\left(m_{K}-m_{\pi}\right)^{2}\right]}}{2 \sqrt{s_{12}}}
$$

and

$$
\tilde{A}\left(B^{-} \rightarrow \bar{K}_{2}^{* 0} \pi^{-}\right)=\frac{G_{F}}{\sqrt{2}} \sum_{p=u, c} \lambda_{p}^{(s)} \frac{m_{K_{2}^{*}}^{2}}{s_{12}}\left[a_{4}^{p}+\cdots+\beta_{3, \mathrm{EW}}^{p}\right]_{\pi K_{2}^{*}} \tilde{X}^{\left(B \pi, \bar{K}_{2}^{*}\right)},
$$

where $\tilde{X}^{\left(B \pi, \bar{K}_{2}^{*}\right)}$ has the same expression as $X^{\left(B \pi, \bar{K}_{2}^{*}\right)}$ except for a replacement of $p_{c}$ by $\tilde{p}_{c}$ and $F_{1}^{B \pi}\left(m_{K_{2}^{*}}^{2}\right)$ by $F_{1}^{B \pi}\left(s_{12}\right)$. Following the previous case, it is straightforward to show that the factorization relation

$$
\Gamma\left(B^{-} \rightarrow \bar{K}_{2}^{* 0} \pi^{-} \rightarrow K^{-} \pi^{+} \pi^{-}\right) \stackrel{\Gamma_{K_{2}^{*} \rightarrow 0}}{\longrightarrow} \Gamma\left(B^{-} \rightarrow \bar{K}_{2}^{* 0} \pi^{-}\right) \mathcal{B}\left(\bar{K}_{2}^{* 0} \rightarrow K^{-} \pi^{+}\right)
$$

holds in the NWA. 
In QCDF, we obtain

$$
\begin{aligned}
\mathcal{B}\left(B^{-} \rightarrow \bar{K}_{2}^{* 0}(1430) \pi^{-}\right)_{\mathrm{QCDF}} & =\left(2.60_{-2.53}^{+9.07}\right) \times 10^{-6}, \\
A_{C P}\left(B^{-}\right. & \left.\rightarrow \bar{K}_{2}^{* 0}(1430) \pi^{-}\right)_{\mathrm{QCDF}}=\left(1.72_{-1.95}^{+2.12}\right) \%,
\end{aligned}
$$

where the decay constants $f_{K_{2}^{*}}=118 \pm 5 \mathrm{MeV}$ and $f_{K_{2}^{*}}^{\perp}=77 \pm 14 \mathrm{MeV}$ at $\mu=1 \mathrm{GeV}$ [25], and the penguin annihilation effects

$$
\beta_{2}^{p}\left(\pi K_{2}^{*}\right)=0.017+0.006 i, \quad\left(\beta_{3}^{p}+\beta_{3, \mathrm{EW}}^{p}\right)\left(\pi K_{2}^{*}\right)=-0.027+0.022 i
$$

have been used. In the narrow width limit, we find that

$$
\mathcal{B}\left(B^{-} \rightarrow \bar{K}_{2}^{* 0}(1430) \pi^{-} \rightarrow K^{-} \pi^{+} \pi^{-}\right)_{\Gamma_{K_{2}^{*}} \rightarrow 0}=0.864 \times 10^{-6} .
$$

Since $\mathcal{B}\left(K_{2}^{* 0}(1430) \rightarrow \pi^{+} \pi^{-}\right)=(0.499 \pm 0.012) \times \frac{2}{3}$ [3], it is seen that the factorization relation Eq. (4.47) is numerically satisfied.

With the finite-width $\Gamma_{K_{2}^{* 0}}^{0}=109 \pm 5 \mathrm{MeV}$, we obtain ${ }^{8}$

$$
\begin{aligned}
\mathcal{B}\left(B^{-} \rightarrow \bar{K}_{2}^{* 0}(1432) \pi^{-} \rightarrow K^{-} \pi^{+} \pi^{-}\right) & =\left(0.89_{-0.19}^{+0.22}\right) \times 10^{-6}, \\
A_{C P}\left(B^{-}\right. & \left.\rightarrow \bar{K}_{2}^{* 0}(1432) \pi^{-} \rightarrow K^{-} \pi^{+} \pi^{-}\right)=(1.711 \pm 0.002) \%,
\end{aligned}
$$

and

$$
\eta_{K_{2}^{*}}^{\mathrm{QCDF}}=0.972 \pm 0.001 \quad(0.715 \pm 0.009)
$$

As for the $\eta_{K_{2}^{*}}$ parameter in the experimental parametrization, we need to consider two possibilities for the angular distribution function: $T_{2}$ in Eq. (3.2) imposed with the transversality condition and $T_{2}^{\prime}$ in Eq. (3.10) without the transversality condition. We thus find

$$
\eta_{K_{2}^{*}}^{\mathrm{EXPP}}=1.053 \pm 0.002, \quad \eta_{K_{2}^{*}}^{\mathrm{EXPP}}=1.031 \pm 0.001 .
$$

Therefore, the transversality condition has little impact on the determination of $\eta_{R}$. The dependence of $\eta_{K_{2}^{*}}$ in QCDF and in experimental parametrization is shown in Fig. 2. Experimentally, the BABAR measurement [12] yields

$$
\mathcal{B}\left(B^{-} \rightarrow \bar{K}_{2}^{* 0}(1430) \pi^{-} \rightarrow K^{-} \pi^{+} \pi^{-}\right)_{\mathrm{expt}}=\left(1.85_{-0.50}^{+0.73}\right) \times 10^{-6} .
$$

Our result of Eq. (4.51) for the branching fraction is consistent with experiment within uncertainties.

Comparing $\eta_{K_{2}^{*}}$ 's with $\eta_{f_{2}}$ 's, it is clear that the proximity of $\eta_{f_{2}}^{\mathrm{QCDF}}$ to unity in QCDF is unexpected, while the deviation of $\eta_{R}$ from unity in the EXPP scenario is barely consistent with the expectation from the ratio of $\Gamma_{R} / m_{R}$ for $R=f_{2}(1270)$ and $K_{2}^{*}(1430)$.

\section{B. Vector mesons}

We take the processes $B^{-} \rightarrow \rho(770) \pi^{-} \rightarrow \pi^{+} \pi^{-} \pi^{-}$and $B^{-} \rightarrow \bar{K}^{* 0}(892) \pi^{-} \rightarrow K^{-} \pi^{+} \pi^{-}$as examples to illustrate the width effects associated with the vector mesons. ${ }^{9}$ It is known that $\rho(770)$ is much broader than $K^{*}(892)$. Therefore, it is expected that the former is subject to a larger width effect.

\footnotetext{
${ }^{8}$ Contrary to the phase space integration in Eq. (4.22) for $B^{-} \rightarrow f_{2} \pi^{-} \rightarrow \pi+\pi^{-} \pi^{-}$, here one should integrate over $s_{12}$ first and then $s_{23}$ owing to a pole structure in $T^{\mathrm{BW}}\left(s_{12}\right)$ at $s_{12}=m_{K_{2}^{*}}^{2}$.

${ }^{9}$ For an early discussion on the decay $B \rightarrow \rho \pi \rightarrow 3 \pi$, see [26].
}

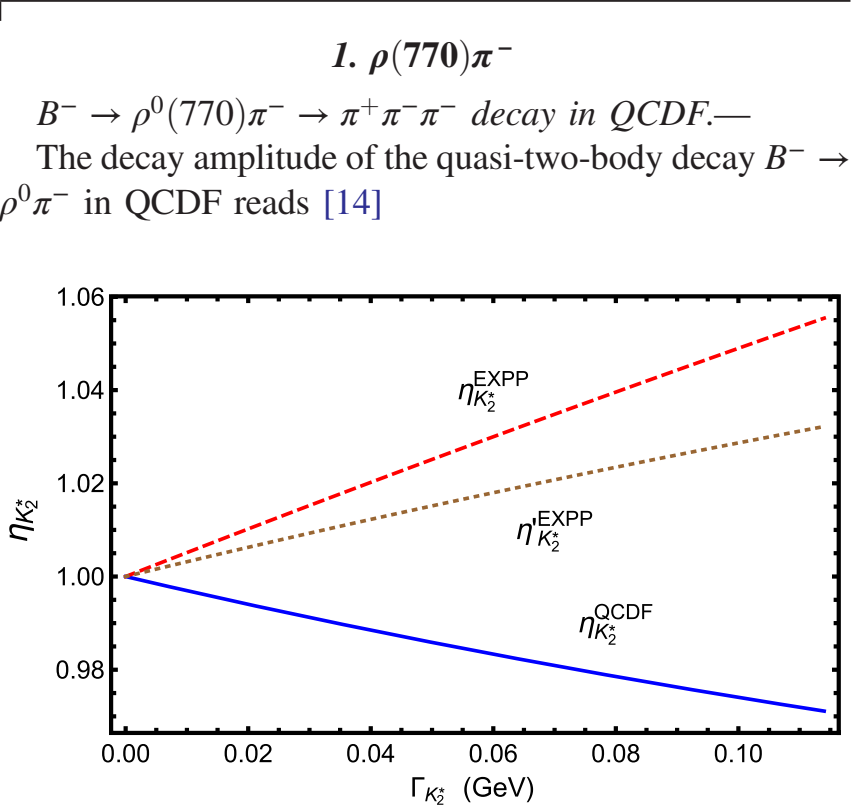

FIG. 2. Same as Fig. 1 for the $\bar{K}_{2}^{*}(1430)$ mediating the $B^{-} \rightarrow$ $K^{-} \pi^{+} \pi^{-}$decay. 


$$
\begin{aligned}
A\left(B^{-} \rightarrow \rho^{0} \pi^{-}\right)= & \frac{G_{F}}{2} \sum_{p=u, c} \lambda_{p}^{(d)}\left\{\left[\delta_{p u}\left(a_{2}-\beta_{2}\right)-a_{4}^{p}-r_{\chi}^{\rho} a_{6}^{p}+\frac{3}{2}\left(a_{7}^{p}+a_{9}^{p}\right)+\frac{1}{2}\left(a_{10}^{p}+r_{\chi}^{\rho} a_{8}^{p}\right)\right.\right. \\
& \left.-\beta_{3}^{p}-\beta_{3, \mathrm{EW}}^{p}\right]_{\pi \rho} X^{\left(B^{-} \pi, \rho\right)}+\left[\delta_{p u}\left(a_{1}+\beta_{2}\right)+a_{4}^{p}-r_{\chi}^{\pi} a_{6}^{p}+a_{10}^{p}-r_{\chi}^{\pi} a_{8}^{p}\right. \\
& \left.\left.+\beta_{3}^{p}+\beta_{3, \mathrm{EW}}^{p}\right]_{\rho \pi} X^{\left(B^{-} \rho, \pi\right)}\right\},
\end{aligned}
$$

with the chiral factor

$$
r_{\chi}^{\rho}(\mu)=\frac{2 m_{\rho}}{m_{b}(\mu)} \frac{f_{\rho}^{\perp}(\mu)}{f_{\rho}}
$$

and the factorizable matrix elements

$$
X^{\left(B^{-} \pi, \rho\right)}=2 f_{\rho} m_{B} p_{c} F_{1}^{B \pi}\left(m_{\rho}^{2}\right), \quad X^{\left(B^{-} \rho, \pi\right)}=2 f_{\pi} m_{B} p_{c} A_{0}^{B \rho}\left(m_{\pi}^{2}\right),
$$

where we have followed [27] for the definitions of $B \rightarrow P$ and $B \rightarrow V$ transition form factors.

The so-called Gounaris-Sakurai model [7] is a popular approach for describing the broad $\rho(770)$ resonance. The line shape is introduced in Eq. (2.25). Note that the GS line shape for $\rho(770)$ was employed by both BABAR [8] and LHCb $[9,10]$ in their analysis of the $\rho(770)$ resonance in the $B^{-} \rightarrow \pi^{+} \pi^{-} \pi^{-}$decay.

For the three-body decay amplitude $\mathcal{A}_{\rho(770)} \equiv A\left(\mathcal{B}^{-} \rightarrow \rho^{0}(770) \pi^{-} \rightarrow \pi^{-}\left(p_{1}\right) \pi^{+}\left(p_{2}\right) \pi^{-}\left(p_{3}\right)\right)$, factorization leads to the expression [15]

$$
\begin{aligned}
\mathcal{A}_{\rho(770)}= & -\frac{G_{F}}{2} \sum_{p=u, c} \lambda_{p}^{(d)} g^{\rho \rightarrow \pi^{+} \pi^{-}} F\left(s_{23}, m_{\rho}\right) T_{\rho}^{\mathrm{GS}}\left(s_{23}\right)\left(s_{12}-s_{13}\right) \\
& \times\left\{f_{\pi}\left[m_{\rho} A_{0}^{B \rho}\left(m_{\pi}^{2}\right)+\frac{1}{2}\left(m_{B}-m_{\rho}-\frac{m_{B}^{2}-s_{23}}{m_{B}+m_{\rho}}\right) A_{2}^{B \rho}\left(m_{\pi}^{2}\right)\right]\right. \\
& \times\left[\delta_{p u}\left(a_{1}+\beta_{2}\right)+a_{4}^{p}-r_{\chi}^{\pi} a_{6}^{p}+a_{10}^{p}-r_{\chi}^{\pi} a_{8}^{p}+\beta_{3}^{p}+\beta_{3, \mathrm{EW}}^{p}\right]_{\rho \pi}+m_{\rho} f_{\rho} F_{1}^{B \pi}\left(s_{23}\right) \\
& \left.\times\left[\delta_{p u}\left(a_{2}-\beta_{2}\right)-a_{4}^{p}-r_{\chi}^{\rho} a_{6}^{p}+\frac{3}{2}\left(a_{7}^{p}+a_{9}^{p}\right)+\frac{1}{2}\left(a_{10}^{p}+r_{\chi}^{\rho} a_{8}^{p}\right)-\beta_{3}^{p}-\beta_{3, \mathrm{EW}}^{p}\right]_{\pi \rho}\right\}+\left(s_{23} \leftrightarrow s_{12}\right) .
\end{aligned}
$$

Penguin annihilation terms characterized by $\beta_{2}, \beta_{3}$, and $\beta_{3 \text {,EW }}$, which are absent in naïve factorization, are included here. Note that

$$
s_{12}-s_{13}=-4 \vec{p}_{1} \cdot \vec{p}_{2}=4 \vec{p}_{1} \cdot \vec{p}_{3}=4\left|\vec{p}_{1}\right|\left|\vec{p}_{3}\right| \cos \theta
$$

in the rest frame of $\pi^{+}\left(p_{2}\right)$ and $\pi^{-}\left(p_{3}\right)$ with the expressions of $\left|\vec{p}_{i}\right|(i=1,2,3)$ given in Eq. (4.14). Then we can write

$$
\mathcal{A}_{\rho(770)}=-g^{\rho \rightarrow \pi^{+} \pi^{-}} F\left(s_{23}, m_{\rho}\right) T_{\rho}^{\mathrm{GS}}\left(s_{23}\right) 2 q \cos \theta \tilde{A}\left(B^{-} \rightarrow \rho \pi^{-}\right)+\left(s_{23} \leftrightarrow s_{12}\right),
$$

with $q$ already introduced in Eq. (4.14), where

$$
\tilde{A}\left(B^{-} \rightarrow \rho \pi^{-}\right)=\frac{G_{F}}{2} \sum_{p=u, c} \lambda_{p}^{(d)}\left\{\left[\delta_{p u}\left(a_{1}+\beta_{2}\right)+a_{4}^{p}+\cdots\right]_{\rho \pi} \tilde{X}^{\left(B^{-} \rho, \pi\right)}+\left[\delta_{p u}\left(a_{2}-\beta_{2}\right)-a_{4}^{p}+\cdots\right]_{\pi \rho} \tilde{X}^{\left(B^{-} \pi, \rho\right)}\right\}
$$

with 


$$
\begin{aligned}
& \tilde{X}^{\left(B^{-} \pi, \rho\right)}=2 f_{\rho} m_{B} \tilde{p}_{c} F_{1}^{B \pi}\left(s_{23}\right), \\
& \tilde{X}^{\left(B^{-} \rho, \pi\right)}=2 f_{\pi} m_{B} \tilde{p}_{c}\left[A_{0}^{B \rho}\left(m_{\pi}^{2}\right)+\frac{1}{2 m_{\rho}}\left(m_{B}-m_{\rho}-\frac{m_{B}^{2}-s_{23}}{m_{B}+m_{\rho}}\right) A_{2}^{B \rho}\left(m_{\pi}^{2}\right)\right] .
\end{aligned}
$$

The decay rate is given by

$$
\begin{aligned}
\Gamma\left(B^{-} \rightarrow \rho \pi^{-} \rightarrow \pi^{+} \pi^{-} \pi^{-}\right)= & \frac{1}{2} \frac{1}{(2 \pi)^{3} 32 m_{B}^{3}} \int d s_{23} d s_{12}\left\{\frac{\left|g^{\rho \rightarrow \pi^{+} \pi^{-}}\right|^{2} F\left(s_{23}, m_{\rho}\right)^{2}\left(1+D \Gamma_{\rho}^{0} / m_{\rho}\right)^{2}}{\left(s_{23}-m_{\rho}^{2}-f\left(s_{23}\right)\right)^{2}+m_{\rho}^{2} \Gamma_{\rho}^{2}\left(s_{23}\right)}\right. \\
& \left.\times 4 q^{2} \cos ^{2} \theta\left|\tilde{A}\left(B^{-} \rightarrow \rho \pi^{-}\right)\right|^{2}+\left(s_{23} \leftrightarrow s_{12}\right)+\text { interference }\right\} .
\end{aligned}
$$

One can integrate out the angular distribution part by noting that

$$
\int_{\left(s_{12}\right)_{\min }}^{\left(s_{12}\right)_{\max }} d s_{12} \cos ^{2} \theta=\frac{2}{3 a}=\frac{4}{3} \frac{m_{B}}{\sqrt{s_{23}}} q \tilde{p}_{c} .
$$

In the narrow width limit,

$$
\frac{m_{\rho} \Gamma_{\rho}(s)\left(1+D \Gamma_{\rho}^{0} / m_{\rho}\right)^{2}}{\left(s-m_{\rho}^{2}-f(s)\right)^{2}+m_{\rho}^{2} \Gamma_{\rho}^{2}(s)} \stackrel{\Gamma_{\rho} \rightarrow 0}{\longrightarrow} \pi \delta\left(s-m_{\rho}^{2}-f(s)\right) .
$$

We see from Eq. (2.27) that $f(s)$ vanishes when $s \rightarrow m_{\rho}^{2}$. Hence, the $\delta$-function implies $s \rightarrow m_{\rho}^{2}$ in the zero width limit. As a result, $\tilde{p}_{c} \rightarrow p_{c}, q \rightarrow q_{0}$, and $\tilde{A}\left(B^{-} \rightarrow \rho \pi^{-}\right) \rightarrow A\left(B^{-} \rightarrow \rho \pi^{-}\right)$. We then obtain the desired factorization relation

$$
\Gamma\left(B^{-} \rightarrow \rho \pi^{-} \rightarrow \pi^{+} \pi^{-} \pi^{-}\right) \stackrel{\Gamma_{\rho} \rightarrow 0}{\longrightarrow} \Gamma\left(B^{-} \rightarrow \rho \pi^{-}\right) \mathcal{B}\left(\rho \rightarrow \pi^{+} \pi^{-}\right),
$$

where use of the relations

$$
\Gamma_{\rho \rightarrow \pi^{+} \pi^{-}}=\frac{q_{0}^{3}}{6 \pi m_{\rho}^{2}} g_{\rho \rightarrow \pi^{+} \pi^{-}}^{2}, \quad \Gamma_{B^{-} \rightarrow \rho \pi^{-}}=\frac{p_{c}}{8 \pi m_{B}^{2}}\left|A\left(B^{-} \rightarrow \rho \pi^{-}\right)\right|^{2}
$$

has been made.

Numerical results.-

To compute the flavor operators $a_{i}^{p}(\rho \pi)$ and $a_{i}^{p}(\pi \rho)$ in QCDF, we need to specify the parameters $\rho_{A, H}$ and $\phi_{A, H}$ for penguin annihilation and hard spectator scattering diagrams. For $B \rightarrow V P$ decays, we use the superscripts " $I$ " and " $f$,"

$$
X_{A}^{i, f}=\ln \left(\frac{m_{B}}{\Lambda_{h}}\right)\left(1+\rho_{A}^{i, f} e^{i \phi_{A}^{i, f}}\right)
$$

to distinguish the gluon emission from the initial- and final-state quarks, respectively. We shall use

$$
\left(\rho_{A}^{i}, \rho_{A}^{f}\right)_{P V}=\left(2.87_{-1.95}^{+0.66}, 0.91_{-0.13}^{+0.12}\right), \quad\left(\phi_{A}^{i}, \phi_{A}^{f}\right)_{P V}=\left(-145_{-21}^{+14},-37_{-9}^{+10}\right)^{\circ}
$$

and the first order approximation of $\rho_{H} \approx \rho_{A}^{i}$ and $\phi_{H} \approx \phi_{A}^{i}$ (see [28] for details). This leads to

$$
\begin{aligned}
& \beta_{2}^{p}(\rho \pi)=0.025+0.011 i, \quad\left(\beta_{3}^{p}+\beta_{3, \mathrm{EW}}^{p}\right)(\rho \pi)=0.034-0.030 i \\
& \beta_{2}^{p}(\pi \rho)=-0.018-0.008 i, \quad\left(\beta_{3}^{p}+\beta_{3, \mathrm{EW}}^{p}\right)(\pi \rho)=0.026-0.021 i
\end{aligned}
$$

and the flavor operators $a_{i}^{p}(\rho \pi)$ and $a_{i}^{p}(\pi \rho)$ shown in Table II. 
TABLE II. Numerical values of the flavor operators $a_{i}^{p}\left(M_{1} M_{2}\right)$ for $M_{1} M_{2}=\rho(770) \pi$ and $\pi \rho(770)$ at the scale $\mu=\bar{m}_{b}\left(\bar{m}_{b}\right)=4.18 \mathrm{GeV}$.

\begin{tabular}{cccccc}
\hline \hline$a_{i}^{p}$ & $\rho \pi$ & $\pi \rho$ & $a_{i}^{p}$ & $\rho \pi$ & $\pi \rho$ \\
\hline$a_{1}$ & $1.007+0.108 i$ & $1.000+0.095 i$ & $a_{6}^{c}$ & $-0.045-0.005 i$ & $-0.013-0.006 i$ \\
$a_{2}$ & $0.135-0.379 i$ & $0.158-0.340 i$ & $a_{7}$ & $(-0.13+2.9 i) 10^{-4}$ & $(-0.3+2.6 i) 10^{-4}$ \\
$a_{3}$ & $0.0008+0.0183 i$ & $-0.0004+0.016 i$ & $a_{8}^{u}$ & $(5.2-1.0 i) 10^{-4}$ & $(-8.9-8.5 i) 10^{-5}$ \\
$a_{4}^{u}$ & $-0.026-0.022 i$ & $-0.026-0.021 i$ & $a_{8}^{c}$ & $(5.0-0.5 i) 10^{-4}$ & $(-10.7-3.7 i) 10^{-5}$ \\
$a_{4}^{c}$ & $-0.030-0.013 i$ & $-0.031-0.012 i$ & $a_{9}$ & $(-9.1-0.9 i) 10^{-3}$ & $(-9.0-0.8 i) 10^{-3}$ \\
$a_{5}$ & $0.0018-0.0247 i$ & $0.004-0.022 i$ & $a_{10}^{u}$ & $(-0.9+3.3 i) 10^{-3}$ & $(-1.1+2.9 i) 10^{-3}$ \\
$a_{6}^{u}$ & $-0.042-0.014 i$ & $-0.010-0.015 i$ & $a_{10}^{c}$ & $(-0.9+3.3 i) 10^{-3}$ & $(-1.2+3.0 i) 10^{-3}$ \\
\hline \hline
\end{tabular}

Following [29], we obtain in QCDF,

$$
\begin{aligned}
\mathcal{B}\left(B^{-} \rightarrow \rho(770) \pi^{-}\right)_{\mathrm{QCDF}} & =\left(8.18_{-0.81}^{+1.67}\right) \times 10^{-6}, \\
A_{C P}\left(B^{-} \rightarrow \rho(770) \pi^{-}\right)_{\mathrm{QCDF}} & =\left(0.36_{-4.54}^{+5.36}\right) \%,
\end{aligned}
$$

where use of the decay constants $f_{\rho}=216 \mathrm{MeV}$ and $f_{\rho}^{\perp}(\mu=1 \mathrm{GeV})=165 \mathrm{MeV}$ [29] has been made. For the finite-width $\Gamma_{\rho}^{0}=149.1 \pm 0.8 \mathrm{MeV}$, we find

$$
\begin{aligned}
\mathcal{B}\left(B^{-} \rightarrow \rho(770) \pi^{-} \rightarrow \pi^{+} \pi^{-} \pi^{-}\right) & =\left(8.76_{-1.68}^{+1.86}\right) \times 10^{-6}, \\
A_{C P}\left(B^{-} \rightarrow \rho(770) \pi^{-} \rightarrow \pi^{+} \pi^{-} \pi^{-}\right) & =-\left(0.24_{-0.54}^{+0.46}\right) \%,
\end{aligned}
$$

and

$$
\eta_{\rho \pi}^{\mathrm{GS}, \mathrm{QCDF}}=0.931 \quad(0.855),
$$

with negligible uncertainties, where the value in parentheses is obtained with $F\left(s, m_{f_{2}}\right)=1$. The same results for $\eta_{\rho \pi}^{\mathrm{GS}, \mathrm{QCDF}}$ can also be obtained using Eqs. (2.31) and (4.60). The deviation of $\eta_{\rho}^{\mathrm{GS}}$ from unity at $7 \%$ level is contrasted with the ratio $\Gamma_{\rho} / m_{\rho}=0.192$. For comparison, using the Breit-Wigner model to describe the $\rho$ line shape, we get

$$
\eta_{\rho \pi}^{\mathrm{BW}, \mathrm{QCDF}}=1.111 \pm 0.001, \quad(1.033)
$$

In the experimental parametrization scheme, we obtain

$$
\eta_{\rho \pi}^{\mathrm{GS}, \mathrm{EXPP}}=0.950, \quad \eta_{\rho \pi}^{\mathrm{BW}, \mathrm{EXPP}}=1.152 \pm 0.001 .
$$

The parameter $\eta_{\rho}$ as a function of the $\rho(770)$ width is shown in Fig. 3 for both Gounaris-Sakurai and BreitWigner line shape models and for both QCDF and EXPP schemes.

As shown in Eq. (2.32), the expression of $\eta_{\rho}^{\mathrm{GS}}$ is the same as that of $\eta_{\rho}^{\mathrm{BW}}$ except for an additional $r^{2} \equiv(1+$ $\left.D \Gamma_{\rho}^{0} / m_{\rho}\right)^{2}$ factor in the denominator. This $r^{2}$ term accounts for the fact that $\eta_{\rho}^{\mathrm{GS}}<1<\eta_{\rho}^{\mathrm{BW}}$ in both QCDF and EXPP schemes. Since the Gounaris-Sakurai line shape was employed by both $B A B A R$ and LHCb Collaborations in their analyses of the $\rho$ resonance in $B^{-} \rightarrow \pi^{+} \pi^{-} \pi^{-}$decay, the branching fraction of $B^{-} \rightarrow \rho \pi^{-}$should be corrected using $\eta_{\rho}^{\mathrm{GS}}$ rather than $\eta_{\rho}^{\mathrm{BW}}$.

From the measured branching fraction $\mathcal{B}\left(B^{-} \rightarrow\right.$ $\left.\rho(770) \pi^{-} \rightarrow \pi^{+} \pi^{-} \pi^{-}\right)=(8.44 \pm 0.87) \times 10^{-6}$ by $\mathrm{LHCb}$ $[9,10]$ and $\left(8.1 \pm 0.7 \pm 1.2_{-1.1}^{+0.4}\right) \times 10^{-6}$ by BABAR [8], we obtain the world average

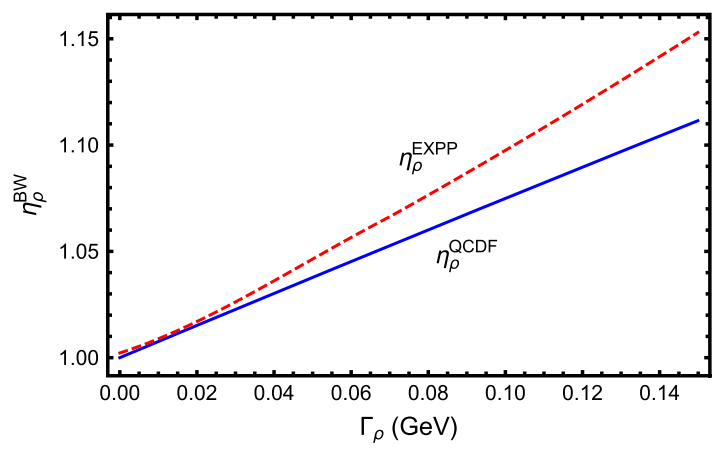

(b)

FIG. 3. Same as Fig. 1 for the $\rho(770)$ resonance mediating the $B^{-} \rightarrow \pi^{+} \pi^{-} \pi^{-}$decay using (a) the Gounaris-Sakurai model and (b) the Breit-Wigner model to describe its line shape. 
$\mathcal{B}\left(B^{-} \rightarrow \rho(770) \pi^{-} \rightarrow \pi^{+} \pi^{-} \pi^{-}\right)_{\operatorname{expt}}=(8.36 \pm 0.77) \times 10^{-6}$.

It is worth emphasizing that the $C P$ asymmetry for the quasi-two-body decay $B^{-} \rightarrow \rho^{0} \pi^{-}$has been found by $\mathrm{LHCb}$ to be consistent with zero in all three $S$-wave approaches. For example, $\mathcal{A}_{C P}\left(\rho^{0} \pi^{-}\right)=(0.7 \pm 1.9) \%$ in the isobar model $[9,10]$. However, previous theoretical predictions all lead to a negative $C P$ asymmetry for
$B^{-} \rightarrow \rho^{0} \pi^{-}$, ranging from $-7 \%$ to $-45 \%$ (see [28] for a detailed discussion). The QCDF results for the branching fraction and $C P$ asymmetry presented in Eq. (4.72) agree with experiment.

\section{2. $\rho(770) K^{-}$}

The three-body decay amplitude $\mathcal{A}_{\rho(770) K^{-}} \equiv A\left(\mathcal{B}^{-} \rightarrow\right.$ $\left.K^{-} \rho(770) \rightarrow K^{-}\left(p_{1}\right) \pi^{+}\left(p_{2}\right) \pi^{-}\left(p_{3}\right)\right)$ has the expression

$$
\begin{aligned}
\mathcal{A}_{\rho(770) K^{-}}= & -\frac{G_{F}}{2} \sum_{p=u, c} \lambda_{p}^{(s)} g^{\rho \rightarrow \pi^{+} \pi^{-}} F\left(s_{23}, m_{\rho}\right) T_{\rho}^{\mathrm{GS}}\left(s_{23}\right)\left(s_{12}-s_{13}\right) \\
& \times\left\{f_{K}\left[m_{\rho} A_{0}^{B \rho}\left(m_{K}^{2}\right)+\frac{1}{2}\left(m_{B}-m_{\rho}-\frac{m_{B}^{2}-s_{23}}{m_{B}+m_{\rho}}\right) A_{2}^{B \rho}\left(m_{K}^{2}\right)\right]\right. \\
& \times\left[\delta_{p u}\left(a_{1}+\beta_{2}\right)+a_{4}^{p}+a_{10}^{p}-r_{\chi}^{K}\left(a_{6}^{p}+a_{8}^{p}\right)+\beta_{3}^{p}+\beta_{3, \mathrm{EW}}^{p}\right]_{\rho K} \\
& \left.+m_{\rho} f_{\rho} F_{1}^{B K}\left(s_{23}\right)\left[\delta_{p u} a_{2}+\frac{3}{2}\left(a_{7}^{p}+a_{9}^{p}\right)\right]_{K \rho}\right\}, \\
= & -g^{\rho \rightarrow \pi^{+} \pi^{-}} F\left(s_{23}, m_{\rho}\right) T_{\rho}^{\mathrm{GS}}\left(s_{23}\right) 2 q \cos \theta \tilde{A}\left(B^{-} \rightarrow \rho K^{-}\right),
\end{aligned}
$$

where use of Eq. (4.59) has been made, and $\tilde{A}\left(B^{-} \rightarrow \rho K^{-}\right)$has the same expression as the QCDF amplitude for the quasitwo-body decay $B^{-} \rightarrow \rho K^{-}$[14],

$$
\begin{aligned}
A\left(B^{-} \rightarrow \rho K^{-}\right)= & \frac{G_{F}}{2} \sum_{p=u, c} \lambda_{p}^{(s)}\left\{2 f_{\rho} m_{B} p_{c} F_{1}^{B K}\left(m_{\rho}^{2}\right)\left[\delta_{p u} a_{2}+\frac{3}{2}\left(a_{7}^{p}+a_{9}^{p}\right)\right]_{K \rho}\right. \\
& \left.+2 f_{K} m_{B} p_{c} A_{0}^{B \rho}\left(m_{K}^{2}\right)\left[\delta_{p u}\left(a_{1}+\beta_{2}\right)+a_{4}^{p}+a_{10}^{p}-r_{\chi}^{K}\left(a_{6}^{p}+a_{8}^{p}\right)+\beta_{3}^{p}+\beta_{3, \mathrm{EW}}^{p}\right]_{\rho K}\right\}
\end{aligned}
$$

except for a replacement of $p_{c} F_{1}^{B K}\left(m_{\rho}^{2}\right)$ by $\tilde{p}_{c} F_{1}^{B K}\left(s_{23}\right)$ and $A_{0}^{B \rho}\left(m_{K}^{2}\right)$ by

$A_{0}^{B \rho}\left(m_{K}^{2}\right)+\frac{1}{2 m_{\rho}}\left(m_{B}-m_{\rho}-\frac{m_{B}^{2}-s_{23}}{m_{B}+m_{\rho}}\right) A_{2}^{B \rho}\left(m_{K}^{2}\right)$.

In QCDF, we obtain

$$
\begin{aligned}
\mathcal{B}\left(B^{-} \rightarrow \rho(770) K^{-}\right)_{\mathrm{QCDF}} & =\left(4.03_{-1.67}^{+3.56}\right) \times 10^{-6}, \\
A_{C P}\left(B^{-}\right. & \left.\rightarrow \rho(770) K^{-}\right)_{\mathrm{QCDF}}=\left(21.6_{-16.6}^{+17.1}\right) \% .
\end{aligned}
$$

For the finite $\rho$ width, we find

$$
\begin{aligned}
\mathcal{B}\left(B^{-} \rightarrow K^{-} \rho(770)\right. & \left.\rightarrow K^{-} \pi^{+} \pi^{-}\right)=\left(4.23_{-0.84}^{+0.95}\right) \times 10^{-6}, \\
A_{C P}\left(B^{-} \rightarrow K^{-} \rho(770)\right. & \left.\rightarrow K^{-} \pi^{+} \pi^{-}\right)=(20.5 \pm 0.8) \%,
\end{aligned}
$$

and

$$
\eta_{\rho K}^{\mathrm{GS}, \mathrm{QCDF}}=0.951 \pm 0.003, \quad(0.899) .
$$

As a comparison, if the Breit-Wigner model is used to describe the $\rho$ line shape, we are led to have

$$
\eta_{\rho K}^{\mathrm{BW}, \mathrm{QCDF}}=1.132 \pm 0.001, \quad(1.086)
$$

In the experimental parametrization scheme, we obtain

$$
\eta_{\rho K}^{\mathrm{GS}, \mathrm{EXPP}}=0.931, \quad \eta_{\rho K}^{\mathrm{BW}, \mathrm{EXPP}}=1.128 \pm 0.001 .
$$

The dependence of $\eta_{\rho}$ as a function of the $\rho(770)$ width is shown in Fig. 4 for both the Gounaris-Sakurai and BreitWigner line shape models. It is evident that $\eta_{\rho \pi}$ and $\eta_{\rho K}$ are close to each other, as it should be. Our predictions in Eq. (4.81) are consistent with the data,

$$
\begin{aligned}
\mathcal{B}\left(B^{-} \rightarrow K^{-} \rho(770) \rightarrow K^{-} \pi^{+} \pi^{-}\right)_{\mathrm{PDG}}= & (3.7 \pm 0.5) \\
& \times 10^{-6}, \\
A_{C P}\left(B^{-} \rightarrow K^{-} \rho(770) \rightarrow K^{-} \pi^{+} \pi^{-}\right)_{\mathrm{PDG}}= & 0.37 \pm 0.10
\end{aligned}
$$




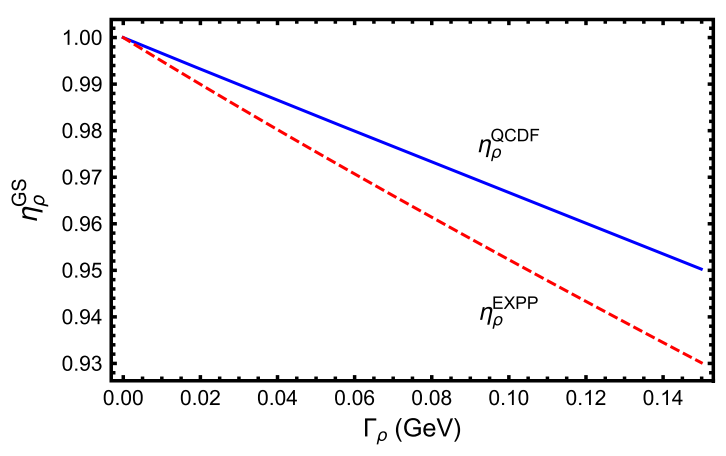

(a)

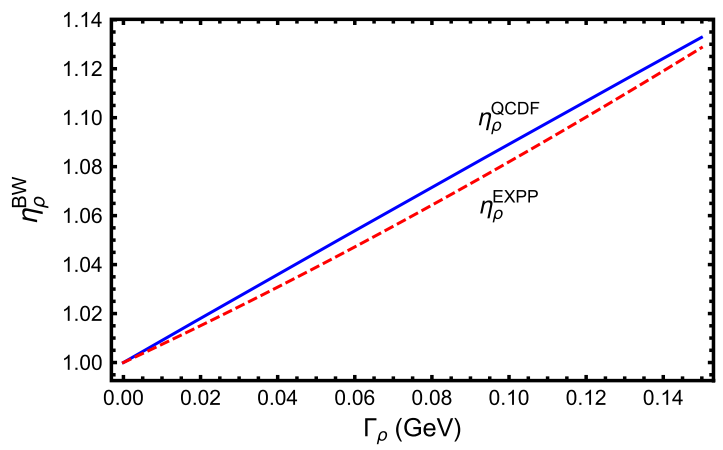

(b)

FIG. 4. Same as Fig. 3 except that the $\rho(770)$ state is the resonance produced in the decay $B^{-} \rightarrow K^{-} \pi^{+} \pi^{-}$.

\section{3. $K^{*}(892)$}

For the three-body decay amplitude $\mathcal{A}_{K^{*}(892)} \equiv A\left(\mathcal{B}^{-} \rightarrow \bar{K}^{* 0}(892) \pi^{-} \rightarrow K^{-}\left(p_{1}\right) \pi^{+}\left(p_{2}\right) \pi^{-}\left(p_{3}\right)\right)$, factorization leads to the expression

$$
\begin{aligned}
\mathcal{A}_{K^{*}(892)}= & -\frac{G_{F}}{\sqrt{2}} \sum_{p=u, c} \lambda_{p}^{(s)} g^{K^{*} \rightarrow K^{-} \pi^{+}} F\left(s_{12}, m_{K^{*}}\right) T_{K^{*}}^{\mathrm{BW}}\left(s_{12}\right)\left[s_{13}-s_{23}-\frac{\left(m_{B}^{2}-m_{\pi}^{2}\right)\left(m_{K}^{2}-m_{\pi}^{2}\right)}{s_{12}}\right] \\
& \times\left[a_{4}^{p}-\frac{1}{2} a_{10}^{p}+r_{\chi}^{K^{*}}\left(a_{6}^{p}-\frac{1}{2} a_{8}^{p}\right)+\delta_{p u} \beta_{2}^{p}+\beta_{3}^{p}+\beta_{3, \mathrm{EW}}^{p}\right]_{\pi K^{*}} m_{K^{*}} f_{K^{*}} F_{1}^{B \pi}\left(s_{12}\right) .
\end{aligned}
$$

Since

$$
s_{13}-s_{23}-\frac{\left(m_{B}^{2}-m_{\pi}^{2}\right)\left(m_{K}^{2}-m_{\pi}^{2}\right)}{s_{12}}=4 \vec{p}_{2} \cdot \vec{p}_{3}=4\left|\vec{p}_{2}\right|\left|\vec{p}_{3}\right| \cos \theta
$$

in the rest frame of $K^{-}\left(p_{1}\right)$ and $\pi^{+}\left(p_{2}\right)$, the three-body amplitude can be recast to

$$
\mathcal{A}_{K^{*}(892)}=-g^{\bar{K}^{*} \rightarrow K^{-} \pi^{+}} F\left(s_{12}, m_{K^{*}}\right) T_{K^{*}}^{\mathrm{BW}}\left(s_{12}\right) 2 q \cos \theta \tilde{A}\left(B^{-} \rightarrow \bar{K}^{* 0}(892) \pi^{-}\right),
$$

where $\tilde{A}\left(B^{-} \rightarrow \bar{K}^{* 0}(892) \pi^{-}\right)$has the same expression as the QCDF amplitude for the quasi-two-body decay $B^{-} \rightarrow$ $\bar{K}^{* 0}(892) \pi^{-}[14]$,

$$
A\left(B^{-} \rightarrow \bar{K}^{* 0} \pi^{-}\right)=\frac{G_{F}}{\sqrt{2}} \sum_{p=u, c} \lambda_{p}^{(s)}\left[a_{4}^{p}-\frac{1}{2} a_{10}^{p}+r_{\chi}^{K^{*}}\left(a_{6}^{p}-\frac{1}{2} a_{8}^{p}\right)+\beta_{2}^{p} \delta_{p u}+\beta_{3}^{p}+\beta_{3, \mathrm{EW}}^{p}\right]_{\pi K^{*}} 2 f_{K^{*}} m_{B} p_{c} F_{1}^{B \pi}\left(m_{K^{*}}^{2}\right),
$$

except for a replacement of $p_{c} F_{1}^{B \pi}\left(m_{K^{*}}^{2}\right)$ by $\tilde{p}_{c} F_{1}^{B \pi}\left(s_{12}\right)$. It is then straightforward to show the factorization relation

$$
\Gamma\left(B^{-} \rightarrow \bar{K}^{* 0}(892) \pi^{-} \rightarrow K^{-} \pi^{+} \pi^{-}\right) \stackrel{\Gamma_{K^{*} \rightarrow 0}}{\longrightarrow} \Gamma\left(B^{-} \rightarrow \bar{K}^{* 0}(892) \pi^{-}\right) \mathcal{B}\left(\bar{K}^{* 0}(892) \rightarrow \pi^{+} \pi^{-}\right)
$$

being valid in the narrow width limit.

In QCDF, we obtain

$$
\begin{aligned}
\mathcal{B}\left(B^{-} \rightarrow \bar{K}^{* 0}(892) \pi^{-}\right) & =\left(10.4_{-1.7}^{+1.8}\right) \times 10^{-6}, \\
A_{C P}\left(B^{-} \rightarrow \bar{K}^{* 0}(892) \pi^{-}\right) & =\left(0.16_{-0.14}^{+0.17}\right) \%,
\end{aligned}
$$

and

$$
\beta_{2}^{p}\left(\pi K^{*}\right)=0.017+0.006 i, \quad\left(\beta_{3}^{p}+\beta_{3, \mathrm{EW}}^{p}\right)\left(\pi K^{*}\right)=-0.027+0.022 i
$$


For the finite-width $\Gamma_{K^{* 0}}^{0}=47.3 \pm 0.5 \mathrm{MeV}$, we find

$$
\begin{aligned}
\mathcal{B}\left(B^{-} \rightarrow \bar{K}^{* 0}(892) \pi^{-} \rightarrow K^{-} \pi^{+} \pi^{-}\right) & =\left(6.52_{-1.42}^{+1.59}\right) \times 10^{-6}, \\
A_{C P}\left(B^{-} \rightarrow \bar{K}^{* 0}(892) \pi^{-} \rightarrow K^{-} \pi^{+} \pi^{-}\right) & =(0.166 \pm 0.002) \%,
\end{aligned}
$$

and

$\eta_{K^{*}}^{\mathrm{BW}, \mathrm{QCDF}}=1.067 \pm 0.002, \quad(0.9914 \pm 0.0001)$.

As for the $\eta_{K^{*}}$ parameter in the experimental parametrization, we obtain

$\eta_{K^{*}}^{\mathrm{EXP}}=1.075 \pm 0.001, \quad \eta_{K^{*}}^{\mathrm{EXPP}}=1.059 \pm 0.001$.

The dependence of $\eta_{K^{*}}$ in QCDF and in experimental parametrization is shown in Fig. 5.

The deviation of $\eta_{K^{*}}$ from unity is roughly consistent with the expectation from the ratio $\Gamma_{K^{*}} / m_{K^{*}}=0.053$. Experimentally, the average of BABAR [12] and Belle [30] measurements yields

$$
\begin{aligned}
\mathcal{B}\left(B^{-} \rightarrow \bar{K}^{* 0}(892) \pi^{-} \rightarrow K^{-} \pi^{+} \pi^{-}\right)_{\operatorname{expt}} \\
\quad=(6.71 \pm 0.57) \times 10^{-6} .
\end{aligned}
$$

The result of the QCDF calculation of the branching fraction given in Eq. (4.93) agrees with experimental data.

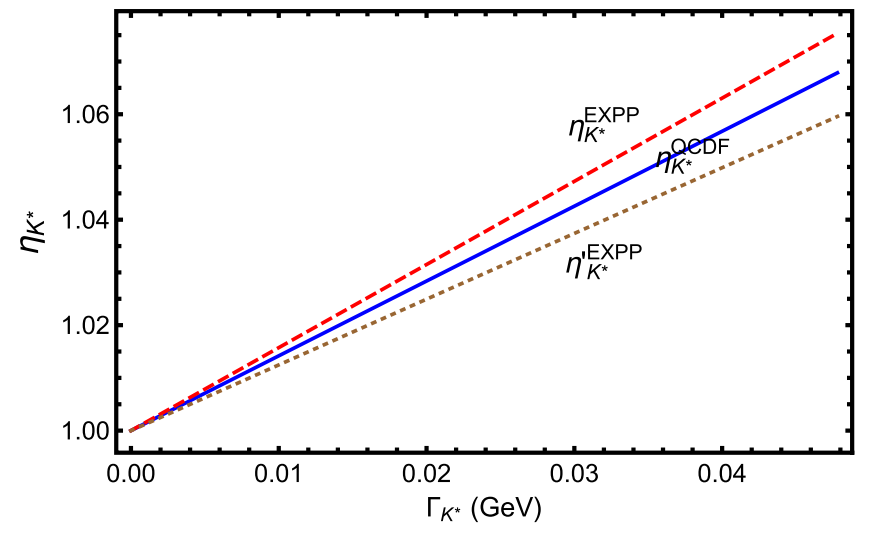

FIG. 5. Same as Fig. 1 for the resonance $K^{*}(892)$ produced in the three-body decay $B^{-} \rightarrow K^{-} \pi^{+} \pi^{-}$.

\section{Scalar resonances}

For examples of scalar intermediate states, we shall take the processes $B^{-} \rightarrow \sigma / f_{0}(500) \pi^{-} \rightarrow \pi^{+} \pi^{-} \pi^{-}$and $B^{-} \rightarrow$ $\bar{K}_{0}^{*}(1430) \pi^{-} \rightarrow K^{-} \pi^{+} \pi^{-}$to illustrate their finite-width effects. Since $K_{0}^{*}(1430)$ and especially $\sigma$ are very broad, they are expected to exhibit large width effects. ${ }^{10}$

\section{1. $\sigma / f_{0}(500)$}

In QCDF, the decay amplitude of $B^{-} \rightarrow \sigma \pi^{-}$is given by (see Eq. (A6) of [32])

$$
\begin{aligned}
A\left(B^{-} \rightarrow \sigma \pi^{-}\right)= & \frac{G_{F}}{\sqrt{2}} \sum_{p=u, c} \lambda_{p}^{(d)}\left\{\left[a_{1} \delta_{p u}+a_{4}^{p}+a_{10}^{p}-\left(a_{6}^{p}+a_{8}^{p}\right) r_{\chi}^{\pi}\right]_{\sigma \pi} X^{(B \sigma, \pi)}\right. \\
& +\left[a_{2} \delta_{p u}+2\left(a_{3}^{p}+a_{5}^{p}\right)+\frac{1}{2}\left(a_{7}^{p}+a_{9}^{p}\right)+a_{4}^{p}-\frac{1}{2} a_{10}^{p}-\left(a_{6}^{p}-\frac{1}{2} a_{8}^{p}\right) \bar{r}_{\chi}^{\sigma}\right]_{\pi \sigma} X^{(B \pi, \sigma)} \\
& \left.-f_{B} f_{\pi} \bar{f}_{\sigma}^{u}\left[\delta_{p u} b_{2}(\pi \sigma)+b_{3}(\pi \sigma)+b_{3, \mathrm{EW}}(\pi \sigma)+(\pi \sigma \rightarrow \sigma \pi)\right]\right\},
\end{aligned}
$$

where the factorizable matrix elements read

$$
X^{(B \sigma, \pi)}=-f_{\pi} F_{0}^{B \sigma^{u}}\left(m_{\pi}^{2}\right)\left(m_{B}^{2}-m_{\sigma}^{2}\right), \quad X^{(B \pi, \sigma)}=\bar{f}_{\sigma}^{u} F_{0}^{B \pi}\left(m_{\sigma}^{2}\right)\left(m_{B}^{2}-m_{\pi}^{2}\right),
$$

and $\bar{r}_{\chi}^{\sigma}(\mu)=2 m_{\sigma} / m_{b}(\mu)$. The superscript $u$ in the scalar decay constant $\bar{f}_{\sigma}^{u}$ and the form factor $F^{B \sigma^{u}}$ refers to the $u$ quark component of the $\sigma$ meson. The scale-dependent scalar decay constant is defined by $\langle\sigma|\bar{u} u| 0\rangle=m_{\sigma} \bar{f}_{\sigma}^{u}$. We follow [28] to take $\bar{f}_{\sigma}^{u}=350 \mathrm{MeV}$ at $\mu=1 \mathrm{GeV}$ and $F_{0}^{B \sigma^{u}}(0)=0.25$, where the Clebsch-Gordon coefficient $1 / \sqrt{2}$ is included in $\bar{f}_{\sigma}^{u}$ and $F_{0}^{B \sigma^{u}}$.

As discussed in Sec. II.E, $\sigma$ is too broad to be described by the usual Breit-Wigner line shape. ${ }^{11}$ We thus follow the LHCb Collaboration [10] to use the simple pole description, ${ }^{12}$

\footnotetext{
${ }^{10}$ The finite-width effect for $\sigma / f_{0}(500)$ had been considered in [31].

${ }^{11}$ Another issue with the Breit-Wigner line shape is that the Breit-Wigner mass and width agree with the pole parameters only if the resonance is narrow.

${ }^{12}$ In the analysis of $B^{0} \rightarrow \bar{D}^{0} \pi^{+} \pi^{-}$decays [33], LHCb has adopted the Bugg model [34] to describe the line shape of $\sigma / f_{0}(500)$. However, the parametrization used in this model is rather complicated and the mass parameter $M \sim 1 \mathrm{GeV}$ is not directly related to the $\sigma$ pole mass. Hence, we shall follow [10] to assume a simple pole model.
} 
$T_{\sigma}(s)=\frac{1}{s-s_{\sigma}}=\frac{1}{s-m_{\sigma}^{2}+\Gamma_{\sigma}^{2}(s) / 4+i m_{\sigma} \Gamma_{\sigma}(s)}$,

with $\sqrt{s_{\sigma}}=m_{\sigma}-i \Gamma_{\sigma} / 2$ and

$$
\Gamma_{\sigma}(s)=\Gamma_{\sigma}^{0}\left(\frac{q}{q_{0}}\right) \frac{m_{\sigma}}{\sqrt{s}} .
$$

Using the isobar description of the $\pi^{+} \pi^{-} S$-wave to fit the $B^{+} \rightarrow \pi^{+} \pi^{-} \pi^{+}$decay data, the LHCb Collaboration found [10]

$$
\sqrt{s_{\sigma}}=(563 \pm 10)-i(350 \pm 13) \mathrm{MeV},
$$

consistent with the PDG value of $\sqrt{s_{\sigma}}=(400-550)-$ $i(200-350) \mathrm{MeV}$ [3].

With $\mathcal{A}_{\sigma} \equiv A\left(B^{-} \rightarrow \sigma \pi^{-} \rightarrow \pi^{+} \pi^{-} \pi^{-}\right), \quad$ factorization leads to [15]

$$
\begin{aligned}
\mathcal{A}_{\sigma}= & \frac{G_{F}}{2} \sum_{p=u, c} \lambda_{p}^{(d)} g^{\sigma \rightarrow \pi^{+} \pi^{-}} F\left(s_{23}, m_{\sigma}\right) T_{\sigma}\left(s_{23}\right)\left\{\tilde{X}^{(B \sigma, \pi)}\left[a_{1} \delta_{p u}+a_{4}^{p}+a_{10}^{p}-\left(a_{6}^{p}+a_{8}^{p}\right) r_{\chi}^{\pi}\right]_{\sigma \pi}\right. \\
& \left.+\tilde{X}^{(B \pi, \sigma)}\left[a_{2} \delta_{p u}+2\left(a_{3}^{p}+a_{5}^{p}\right)+\frac{1}{2}\left(a_{7}^{p}+a_{9}^{p}\right)+a_{4}^{p}-\frac{1}{2} a_{10}^{p}-\left(a_{6}^{p}-\frac{1}{2} a_{8}^{p}\right) \bar{r}_{\chi}^{\sigma}\right]_{\pi \sigma}\right\} \\
& +\left(s_{23} \leftrightarrow s_{12}\right) \\
= & g^{\sigma \rightarrow \pi^{+} \pi^{-}} F\left(s_{23}, m_{\sigma}\right) T_{\sigma}\left(s_{23}\right) \tilde{A}\left(B^{-} \rightarrow \sigma \pi^{-}\right)+\left(s_{23} \leftrightarrow s_{12}\right),
\end{aligned}
$$

with

$$
\tilde{X}^{(B \sigma, \pi)}=-f_{\pi}\left(m_{B}^{2}-s_{23}\right) F_{0}^{B \sigma^{u}}\left(m_{\pi}^{2}\right), \quad \tilde{X}^{(B \pi, \sigma)}=\bar{f}_{\sigma}^{u}\left(m_{B}^{2}-m_{\pi}^{2}\right) F_{0}^{B \pi}\left(s_{23}\right) .
$$

Its decay rate reads

$$
\begin{aligned}
\Gamma\left(B^{-} \rightarrow \sigma \pi^{-} \rightarrow \pi^{+} \pi^{-} \pi^{-}\right)= & \frac{1}{2} \frac{1}{(2 \pi)^{3} 32 m_{B}^{3}} \int d s_{23} d s_{12}\left\{\frac{\left|g^{\sigma \rightarrow \pi^{+} \pi^{-}}\right|^{2} F\left(s_{23}, m_{\sigma}\right)^{2}}{\left(s_{23}-m_{\sigma}^{2}+\Gamma_{\sigma}\left(s_{23}\right) / 4\right)^{2}+m_{\sigma}^{2} \Gamma_{\sigma}^{2}\left(s_{23}\right)}\right. \\
& \left.\times\left|\tilde{A}\left(B^{-} \rightarrow \sigma \pi^{-}\right)\right|^{2}+\left(s_{23} \leftrightarrow s_{12}\right)+\text { interference }\right\} .
\end{aligned}
$$

Note that

$$
\int_{\left(s_{12}\right)_{\min }}^{\left(s_{12}\right)_{\max }} d s_{12}=\frac{2}{a}=4 \frac{m_{B}}{\sqrt{s_{23}}} q \tilde{p}_{c} .
$$

Applying the relations

$$
\begin{aligned}
& \Gamma_{\sigma \rightarrow \pi^{+} \pi^{-}}=\frac{q_{0}}{8 \pi m_{\sigma}^{2}} g_{\sigma \rightarrow \pi^{+} \pi^{-}}^{2}, \\
& \Gamma_{B^{-} \rightarrow \sigma \pi^{-}}=\frac{p_{c}}{8 \pi m_{B}^{2}}\left|A\left(B^{-} \rightarrow \sigma \pi^{-}\right)\right|^{2},
\end{aligned}
$$

we arrive at the desired factorization relation ${ }^{13}$

\footnotetext{
${ }^{13}$ In the $\mathrm{LHCb}$ paper, the square of the pole position is defined by $\sqrt{s_{\sigma}}=m_{\sigma}-i \Gamma_{\sigma}$ rather than $m_{\sigma}-i \Gamma_{\sigma} / 2$. In this case, the lefthand side of the factorization relation in Eq. (4.107) should be multiplied by a factor of 2 .
}

$\Gamma\left(B^{-} \rightarrow \sigma \pi^{-} \rightarrow \pi^{+} \pi^{-} \pi^{-}\right) \stackrel{\Gamma_{\sigma} \rightarrow 0}{\longrightarrow} \Gamma\left(B^{-} \rightarrow \sigma \pi^{-}\right) \mathcal{B}\left(\sigma \rightarrow \pi^{+} \pi^{-}\right)$.

Using the input parameters given in [15], we obtain

$$
\begin{aligned}
\mathcal{B}\left(B^{-} \rightarrow \sigma \pi^{-}\right)_{\mathrm{QCDF}} & =\left(5.31_{-0.19-1.18-1.33}^{+0.20+1.33+0.89}\right) \times 10^{-6}, \\
A_{C P}\left(B^{-} \rightarrow \sigma \pi^{-}\right)_{\mathrm{QCDF}} & =\left(15.06_{-0.29-0.03-11.34}^{+0.30+0.02+8.34}\right) \%
\end{aligned}
$$

in QCDF. For the finite-width $\Gamma_{\sigma}^{0}=700 \pm 26 \mathrm{MeV}$, we find

$$
\begin{aligned}
\mathcal{B}\left(B^{-} \rightarrow \sigma \pi^{-} \rightarrow \pi^{+} \pi^{-} \pi^{-}\right) & =\left(1.65_{-0.37}^{+0.42}\right) \times 10^{-6}, \\
A_{C P}\left(B^{-} \rightarrow \sigma \pi^{-} \rightarrow \pi^{+} \pi^{-} \pi^{-}\right) & =(14.7 \pm 0.1) \%,
\end{aligned}
$$

and

$$
\begin{array}{ll}
\eta_{\sigma}^{\mathrm{QCDF}} & =2.15 \pm 0.05 \quad(1.629 \pm 0.025), \\
\eta_{\sigma}^{\mathrm{EXPP}} & =1.64 \pm 0.03
\end{array}
$$




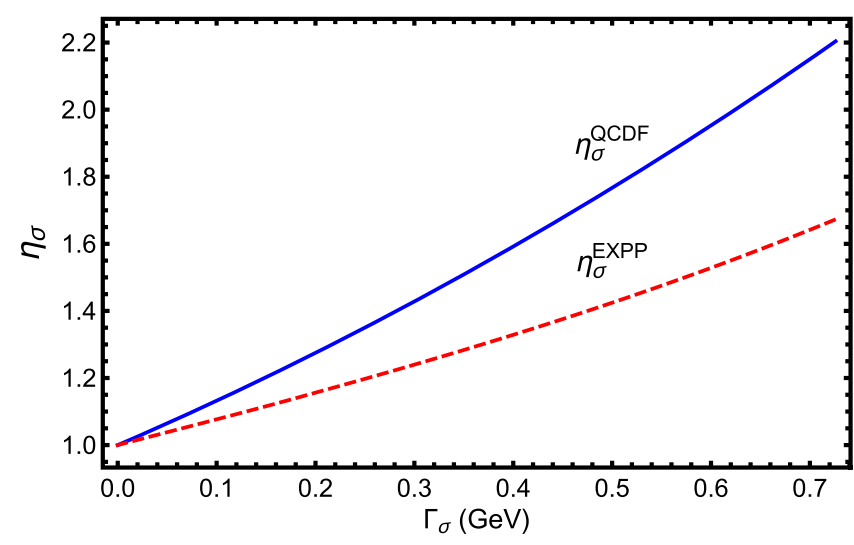

FIG. 6. The parameter $\eta_{\sigma}$ as a function of the $\sigma$ width, where the solid curve is derived from the QCDF calculation and the dashed curve from the EXPP.

where use of Eq. (2.37) has been made for the calculation of $\eta_{\sigma}^{\mathrm{EXPP}}$. The dependence of $\eta_{\sigma}$ on the $\sigma$ width is shown in
Fig. 6. Thus, the width correction is very large here. In Sec. V. B, we shall discuss its implications.

The $\mathrm{LHCb}$ measurement analyzed in the isobar model $[9,10]$ yields

$$
\begin{gathered}
\mathcal{B}\left(B^{-} \rightarrow \sigma \pi^{-} \rightarrow \pi^{+} \pi^{-} \pi^{-}\right)_{\text {expt }}=(3.83 \pm 0.84) \times 10^{-6}, \\
A_{C P}\left(B^{-} \rightarrow \sigma \pi^{-} \rightarrow \pi^{+} \pi^{-} \pi^{-}\right)_{\text {expt }}=\left(14.9_{-0.6}^{+0.5}\right) \%
\end{gathered}
$$

We see that while the calculated $C P$ asymmetry in Eq. (4.109) based on QCDF is in excellent agreement with experiment, the predicted branching fraction is smaller than the measurement by a factor of about 2 .

\section{2. $K_{\mathbf{0}}^{*}(1430)$}

For the three-body decay amplitude $\mathcal{A}_{K_{0}^{*}(1430)} \equiv$ $A\left(B^{-} \rightarrow \bar{K}_{0}^{*}(1430)^{0} \pi^{-} \rightarrow K^{-}\left(p_{1}\right) \pi^{+}\left(p_{2}\right) \pi^{-}\left(p_{3}\right)\right)$, factorization leads to the expression

$$
\begin{aligned}
\mathcal{A}_{K_{0}^{*}(1430)}= & \frac{G_{F}}{\sqrt{2}} \sum_{p=u, c} \lambda_{p}^{(s)} g^{K_{0}^{*} \rightarrow K^{-} \pi^{+}} F\left(s_{12}, m_{K_{0}^{*}}\right) T_{K_{0}^{*}}^{\mathrm{BW}}\left(s_{12}\right)\left[a_{4}^{p}-\frac{1}{2} a_{10}^{p}-r_{\chi}^{K_{0}^{*}}\left(\frac{s_{12}}{m_{K_{0}^{*}}^{2}}\right)\left(a_{6}^{p}-\frac{1}{2} a_{8}^{p}\right)\right. \\
& \left.+\delta_{p u} \beta_{2}^{p}+\beta_{3}^{p}+\beta_{3, \mathrm{EW}}^{p}\right]_{\pi K^{*}} f_{\bar{K}_{0}^{*}} F_{0}^{B \pi}\left(s_{12}\right)\left(m_{B}^{2}-m_{\pi}^{2}\right) \\
= & g^{K_{0}^{*} \rightarrow K^{-} \pi^{+}} F\left(s_{12}, m_{K_{0}^{*}}\right) T_{K_{0}^{*}}^{\mathrm{BW}}\left(s_{12}\right) \tilde{A}\left(B^{-} \rightarrow \bar{K}_{0}^{*}(1430)^{0} \pi^{-}\right),
\end{aligned}
$$

where

$$
r_{\chi}^{K_{0}^{*}}(\mu)=\frac{2 m_{K_{0}^{*}}^{2}}{m_{b}(\mu)\left(m_{s}(\mu)-m_{q}(\mu)\right)},
$$

and the vector decay constant of $\bar{K}_{0}^{*}(1430)$ is related to the scalar one defined by $\left\langle\bar{K}_{0}^{*}|\bar{s} d| 0\right\rangle=m_{K_{0}^{*}} \bar{f}_{\bar{K}_{0}^{*}}$ via $^{14}$

$$
f_{\bar{K}_{0}^{*}}=\frac{m_{s}(\mu)-m_{q}(\mu)}{m_{K_{0}^{*}}} \bar{f}_{\bar{K}_{0}^{*}} .
$$

In QCDF, the decay amplitude of $B^{-} \rightarrow \bar{K}_{0}^{* 0} \pi^{-}$reads [32]

$$
\begin{aligned}
A\left(B^{-} \rightarrow \bar{K}_{0}^{* 0} \pi^{-}\right)= & \left.\frac{G_{F}}{\sqrt{2}} \sum_{p=u, c} \lambda_{p}^{(s)}\left[a_{4}^{p}-r_{\chi}^{K_{0}^{*}} a_{6}^{p}-\frac{1}{2}\left(a_{10}^{p}-r_{\chi}^{K_{0}^{*}} a_{8}^{p}\right)+\delta_{p u} \beta_{2}^{p}+\beta_{3}^{p}+\beta_{3, \mathrm{EW}}^{p}\right)\right]_{\pi K_{0}^{*}} \\
& \times f_{\bar{K}_{0}^{*}} F_{0}^{B \pi}\left(m_{K_{0}^{*}}^{2}\right)\left(m_{B}^{2}-m_{\pi}^{2}\right) .
\end{aligned}
$$

It is obvious that $\tilde{A}\left(B^{-} \rightarrow \bar{K}_{0}^{*}(1430)^{0} \pi^{-}\right)$has the same expression as $A\left(B^{-} \rightarrow \bar{K}_{0}^{* 0} \pi^{-}\right)$except that the chiral factor $r_{\chi}^{K_{0}^{*}}$ is multiplied by a factor of $s_{12} / m_{K_{0}^{*}}^{2}$ (see also [36]) and the form factor $F_{0}^{B \pi}\left(m_{K_{0}^{*}}^{2}\right)$ is replaced by $F_{0}^{B \pi}\left(s_{12}\right)$. As before, we have the factorization relation

$$
\Gamma\left(B^{-} \rightarrow \bar{K}_{0}^{* 0} \pi^{-} \rightarrow K^{-} \pi^{+} \pi^{-}\right) \stackrel{\Gamma_{K_{0}^{*} \rightarrow 0}}{\longrightarrow} \Gamma\left(B^{-} \rightarrow \bar{K}_{0}^{* 0} \pi^{-}\right) \mathcal{B}\left(\bar{K}_{0}^{* 0} \rightarrow \pi^{+} \pi^{-}\right) .
$$

\footnotetext{
${ }^{14}$ The decay constants of a scalar meson and its antiparticle are related by $\bar{f}_{\bar{S}}=\bar{f}_{S}$ and $f_{\bar{S}}=-f_{S}$ [35]. Hence, the vector decay constants of $K_{0}^{*}(1430)$ and $\bar{K}_{0}^{*}(1430)$ are of opposite signs. Using the QCD sum rule result for $\bar{f}_{\bar{K}_{0}^{*}}$ [32], we obtain $f_{\bar{K}_{0}^{*}(1430)}=36.4 \mathrm{MeV}$.
} 


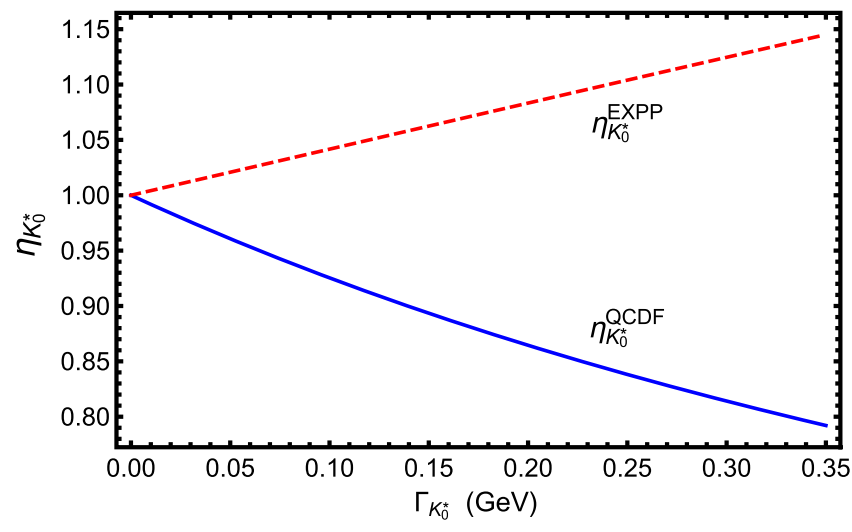

FIG. 7. Same as Fig. 6 for the resonance $\bar{K}_{0}^{*}(1430)$ produced in the three-body decay $B^{-} \rightarrow K^{-} \pi^{+} \pi^{-}$.

Following [32,35], we obtain

$\beta_{2}^{p}\left(\pi K_{0}^{*}\right)=-0.0969, \quad\left(\beta_{3}^{p}+\beta_{3, \mathrm{EW}}^{p}\right)\left(\pi K_{0}^{*}\right)=-0.0323$,

and

$$
\begin{aligned}
\mathcal{B}\left(B^{-} \rightarrow \bar{K}_{0}^{*}(1430)^{0} \pi^{-}\right) & =\left(13.6_{-9.3}^{+39.9}\right) \times 10^{-6}, \\
A_{C P}\left(B^{-} \rightarrow \bar{K}_{0}^{*}(1430)^{0} \pi^{-}\right) & =\left(1.27_{-4.75}^{+5.84}\right) \% .
\end{aligned}
$$

For the finite-width $\Gamma_{K_{0}^{*}(1430)}=270 \pm 80 \mathrm{MeV}$, we find

$$
\begin{aligned}
\mathcal{B}\left(B^{-} \rightarrow \bar{K}_{0}^{*}(1430)^{0} \pi^{-} \rightarrow K^{-} \pi^{+} \pi^{-}\right) & =\left(10.2_{-2.3}^{+3.0}\right) \times 10^{-6}, \\
A_{C P}\left(B^{-} \rightarrow \bar{K}_{0}^{*}(1430)^{0} \pi^{-} \rightarrow K^{-} \pi^{+} \pi^{-}\right) & =(1.12 \pm 0.01) \%,
\end{aligned}
$$

and

$$
\begin{aligned}
& \eta_{K_{0}^{*}}^{\mathrm{QCDF}}=0.83 \pm 0.04, \quad\left(0.31_{-0.05}^{+0.08}\right), \\
& \eta_{K_{0}^{*}}^{\mathrm{EXPP}}=1.11 \pm 0.03
\end{aligned}
$$

The dependence of $\eta_{K_{0}^{*}}$ on the $K_{0}^{*}(1430)$ width in the BreitWigner model is shown in Fig. 7. When off-shell effects on the strong coupling $g^{\bar{K}_{0}^{*} \rightarrow K^{-} \pi^{+}}$are turned off, $\eta_{K_{0}^{*}}^{\mathrm{QCDF}}$ is of order 0.30 , rendering an extremely large deviation from unity, even much larger than $\eta_{\sigma}$. Off-shell effects are particularly significant in this mode because the seemingly large QCDF enhancement in the large $s_{12}$ region is suppressed by the form factor $F\left(s_{12}, m_{K_{0}^{*}}^{2}\right)$. As a consequence, $\eta_{K_{0}^{*}}^{\mathrm{QCDF}}$ becomes about 0.83 .

It has been argued that the Breit-Wigner parametrization is not appropriate for describing the broad $K_{0}^{*}(1430)$ resonance. LASS line shape is an alternative and popular description of the $K_{0}^{*}(1430)$ component proposed by the
TABLE III. Branching fractions (in units of $10^{-6}$ ) of resonant and nonresonant (NR) contributions to $B^{-} \rightarrow K^{-} \pi^{+} \pi^{-}$. Note that the $B A B A R$ 's branching fraction $\left(2.4 \pm 0.5_{-1.5}^{+1.3}\right) \times 10^{-6}$ given in Table II of [12] is for the phase-space nonresonant contribution to $B^{-} \rightarrow K^{-} \pi^{+} \pi^{-}$.

\begin{tabular}{lrc}
\hline \hline Decay mode & $B A B A R[12]$ & Belle [30] \\
\hline $\bar{K}_{0}^{* 0}(1430) \pi^{-}$ & $19.8 \pm 0.7 \pm 1.7_{-0.9}^{+5.6} \pm 3.2$ & $32.0 \pm 1.0 \pm 2.4_{-1.9}^{+1.1}$ \\
$\mathrm{NR}$ & $9.3 \pm 1.0 \pm 1.2_{-0.4}^{+6.7} \pm 1.2$ & $16.9 \pm 1.3 \pm 1.3_{-0.9}^{+1.1}$ \\
\hline \hline
\end{tabular}

LASS Collaboration [37]. In the analysis of three-body decays of $B$ mesons, $B A B A R$ and Belle often adopt different definitions for the $K_{0}^{*}(1430)$ resonance and nonresonant. While Belle (see, e.g., [30]) employed the relativistic BreitWigner model to describe the line shape of the $K_{0}^{*}(1430)$ resonance and an exponential parametrization for the nonresonant contribution, BABAR [12] used the LASS parametrization to describe the elastic $K \pi S$-wave and the $K_{0}^{*}(1430)$ resonance by a single amplitude [37]

$T_{K_{0}^{*}}^{\mathrm{LASS}}(s)=\frac{\sqrt{s}}{q \cot \delta_{B}-i q}-e^{2 i \delta_{B}} \frac{m_{0} \Gamma_{0} \frac{m_{0}}{q_{0}}}{s-m_{0}^{2}+i m_{0} \Gamma_{0} \frac{q}{q_{0}} \frac{m_{0}}{\sqrt{s}}}$,

with

$$
\cot \delta_{B}=\frac{1}{a q}+\frac{1}{2} r q
$$

where $q$ is the c.m. momentum of $K^{-}$and $\pi^{+}$in the $K_{0}^{*}(1430)$ rest frame and $q_{0}$ is the value of $q$ when $s=m_{K_{0}^{*}}^{2}$. The second term of $T_{K_{0}^{*}}^{\mathrm{LASS}}$ is similar to the relativistic BreitWigner function $T_{K_{0}^{*}}^{\mathrm{BW}}$ except for a phase factor $\delta_{B}$ introduced to retain unitarity. The first term is a slowly varying nonresonant component.

The nonresonant branching fraction $\left(2.4 \pm 0.5_{-1.5}^{+1.3}\right) \times$ $10^{-6}$ in $B^{-} \rightarrow K^{-} \pi^{+} \pi^{-}$reported by $B A B A R$ [12] is much smaller than $\left(16.9 \pm 1.3_{-1.6}^{+1.7}\right) \times 10^{-6}$ measured by Belle (see Table III). In the $B A B A R$ analysis, the nonresonant component of the Dalitz plot is modeled as a constant complex phase-space amplitude. Since the first part of the LASS line shape is really nonresonant, it should be added to the phase-space nonresonant piece to get the total nonresonant contribution. Indeed, by combining coherently the nonresonant part of the LASS parametrization and the phase-space nonresonant, $B A B A R$ found the total nonresonant branching fraction to be $\left(9.3 \pm 1.0 \pm 1.2_{-1.3}^{+6.8}\right) \times 10^{-6}$. Evidently, the BABAR result is now consistent with Belle within errors. For the resonant contributions from $K_{0}^{*}(1430)$, the $B A B A R$ results were obtained from $(K \pi)_{0}^{* 0} \pi^{-}$by subtracting the elastic range term from the $K \pi S$-wave [12], namely, the Breit-Wigner component of 
TABLE IV. A summary of the $\eta_{R}$ parameter for various resonances produced in the three-body $B$ decays. Off-shell effects on the strong coupling $g^{R \rightarrow h_{1} h_{2}}$ are taken into account in the determination of $\eta_{R}^{\mathrm{QCDF}}$ but not in $\bar{\eta}_{R}^{\mathrm{QCDF}}$. Uncertainties in $\eta_{R}$ are not specified whenever negligible.

\begin{tabular}{llcclll}
\hline \hline Resonance & $B^{+} \rightarrow R h_{3} \rightarrow h_{1} h_{2} h_{3}$ & $\Gamma_{R}(\mathrm{MeV})[3]$ & $\Gamma_{R} / m_{R}$ & \multicolumn{1}{c}{$\bar{\eta}_{R}^{\text {QCDF }}$} & \multicolumn{1}{c}{$\eta_{R}^{\text {QCDF }}$} & \multicolumn{1}{c}{$\eta_{R}^{\text {EXPP }}$} \\
\hline$f_{2}(1270)$ & $B^{+} \rightarrow f_{2} \pi^{+} \rightarrow \pi^{+} \pi^{-} \pi^{+}$ & $186.7_{-2.5}^{+2.2}$ & 0.146 & 0.974 & $1.003_{-0.002}^{+0.001}$ & $0.937_{-0.005}^{+0.006}$ \\
$K_{2}^{*}(1430)$ & $B^{+} \rightarrow K_{2}^{* 0} \pi^{+} \rightarrow K^{+} \pi^{-} \pi^{+}$ & $109 \pm 5$ & 0.076 & $0.715 \pm 0.009$ & $0.972 \pm 0.001$ & $1.053 \pm 0.002$ \\
$\rho(770)$ & $B^{+} \rightarrow \rho^{0} \pi^{+} \rightarrow \pi^{+} \pi^{-} \pi^{+}$ & $149.1 \pm 0.8$ & 0.192 & $0.86(\mathrm{GS})$ & $0.93(\mathrm{GS})$ & $0.95(\mathrm{GS})$ \\
& & & & $1.03(\mathrm{BW})$ & $1.11(\mathrm{BW})$ & 1.15 (BW) \\
$\rho(770)$ & $B^{+} \rightarrow K^{+} \rho^{0} \rightarrow K^{+} \pi^{+} \pi^{-}$ & $149.1 \pm 0.8$ & 0.192 & $0.90(\mathrm{GS})$ & $0.95(\mathrm{GS})$ & 0.93 (GS) \\
& & & & $1.09(\mathrm{BW})$ & 1.13 (BW) & 1.13 (BW) \\
$K^{*}(892)$ & $B^{+} \rightarrow K^{* 0} \pi^{+} \rightarrow K^{+} \pi^{-} \pi^{+}$ & $47.3 \pm 0.5$ & 0.053 & 1.01 & $1.067 \pm 0.002$ & 1.075 \\
$\sigma / f_{0}(500)$ & $B^{+} \rightarrow \sigma \pi^{+} \rightarrow \pi^{+} \pi^{-} \pi^{+}$ & $700 \pm 26[10]$ & $\approx 1.24$ & $1.63 \pm 0.03$ & $2.15 \pm 0.05$ & $1.64 \pm 0.03$ \\
$K_{0}^{*}(1430)$ & $B^{+} \rightarrow K_{0}^{* 0} \pi^{+} \rightarrow K^{+} \pi^{-} \pi^{+}$ & $270 \pm 80$ & $\approx 0.19$ & $0.31_{-0.05}^{+0.08}$ & $0.83 \pm 0.04$ & $1.11 \pm 0.03$ \\
\hline \hline
\end{tabular}

the LASS parametrization. ${ }^{15}$ Although both $B A B A R$ and Belle employed the Breit-Wigner model to describe the line shape of $K_{0}^{*}(1430)$, the discrepancy between $B A B A R$ and Belle for the $K_{0}^{*} \pi$ mode remains an issue to be resolved.

Note that our calculation of $\mathcal{B}\left(B^{-} \rightarrow \bar{K}_{0}^{*}(1430)^{0} \pi^{-} \rightarrow\right.$ $K^{-} \pi^{+} \pi^{-}$) in Eq. (4.119) based on QCDF is smaller by a factor of 2 (3) when compared to the $B A B A R$ (Belle) measurement. If we follow PDG [3] to apply the naïve factorization relation (1.1), we will obtain using Table III the branching fraction of $B^{-} \rightarrow \bar{K}_{0}^{*}(1430) \pi^{-}$to be $(32.0 \pm$ $\left.1.2_{-6.0}^{+10.8}\right) \times 10^{-6}$ from $B A B A R^{16}$ and $\left(51.6 \pm 1.7_{-7.5}^{+7.0}\right) \times$ $10^{-6}$ from Belle. Obviously, they are much larger than the QCDF prediction given in Eq. (4.118). Indeed, as pointed out before [32,35], this has been a long-standing puzzle that for scalar resonances produced in $B$ decays, the QCDF predictions of $B^{-} \rightarrow \bar{K}_{0}^{* 0}(1430) \pi^{-}$and $\bar{B}^{0} \rightarrow$ $K_{0}^{*-}(1430) \pi^{+}$are in general too small compared to experiment by a factor of $2 \sim 4$. Nevertheless, when the finitewidth effect is taken into account, the PDG values of $\mathcal{B}\left(B^{-} \rightarrow \bar{K}_{0}^{* 0}(1430) \pi^{-}\right)$should be reduced by multiplying a factor of $\eta_{K_{0}^{*}}^{\mathrm{QCDF}} \simeq 0.83$ or further enhanced by a factor of $\eta_{K_{0}^{*}}^{\mathrm{EXPP}} \simeq 1.10$, depending on the scheme.

\section{DISCUSSIONS}

\section{A. Finite-width and off-shell effects}

In Table IV, we give a summary of the $\eta_{R}$ parameters calculated using QCDF and the experimental parametrization for various resonances produced in the three-body $B$ decays. Since the strong coupling of $R\left(m_{12}\right) \rightarrow P_{1} P_{2}$ will be suppressed by the form factor $F\left(s_{12}, m_{R}\right)$ when $m_{12}$ is off shell from $m_{R}$ [see Eq. (4.20)], this implies a

\footnotetext{
${ }^{15}$ It should be stressed that the Breit-Wigner component of the LASS parametrization does not lead to the factorization relation Eq. $_{i 6}$ (4.116).

${ }^{16}$ Another $B A B A R$ measurement of $B^{+} \rightarrow K_{0}^{* 0} \pi^{+} \rightarrow K_{S}^{0} \pi^{0} \pi^{+}$ [38] yields $\mathcal{B}\left(B^{+} \rightarrow K_{0}^{*}(1430) \pi^{+}\right)_{\mathrm{NWA}}=(34.6 \pm 3.3 \pm 4.6) \times 10^{-6}$.
}

suppression of the three-body decay rate in the presence of off-shell effects. Therefore, $\eta_{R}^{\mathrm{QCDF}}$ is always larger than $\bar{\eta}_{R}^{\mathrm{QCDF}}$, with the latter defined for $F\left(s, m_{R}\right)=1$. We see from Table IV that off-shell effects are small in vector meson productions, but prominent in the $K_{2}^{*}(1430)$, $\sigma / f_{0}(500)$, and $K_{0}^{*}(1430)$ resonances. Also, the parameters $\eta_{R}^{\mathrm{QCDF}}$ and $\eta_{R}^{\mathrm{EXPP}}$ are similar for vector mesons, but different for tensor and scalar resonances. To understand the origin of their differences, we need to study the differential decay rates.

In Fig. 8, we show the normalized differential rates of the $B^{-} \rightarrow R \pi^{-} \rightarrow K^{-} \pi^{+} \pi^{-}$and $B^{-} \rightarrow K^{-} R \rightarrow K^{-} \pi^{+} \pi^{-}$decays with $R=\bar{K}^{* 0}(980), \bar{K}_{0}^{* 0}(1430), \bar{K}_{2}^{0}(1430)$, and $\rho^{0}$, respectively, in the left plots. The plots blown up in the resonance regions are also shown in the right plots. Note that the figures on the right are scaled by a factor of $(\pi / 2) \Gamma_{R}$ or $\left(\pi / 2 r^{2}\right) \Gamma_{\rho}^{0}$ with $r \equiv\left(1+D \Gamma_{\rho}^{0} / m_{\rho}\right)$. For the $B^{-} \rightarrow K^{-} \rho^{0} \rightarrow$ $K^{-} \pi^{+} \pi^{-}$decay, we only show the result using the Gounaris-Sakurai line shape, as this is employed by the experimental parametrization for the $\rho$ resonance. The normalized differential rates obtained from the QCDF calculation and the experimental parametrization are shown in the plots. For $R=\bar{K}^{* 0}$ and $\bar{K}_{2}^{0}$, we also show the results using the experimental parametrization with or without enforcing the transversality condition [see Eqs. (3.2) and (3.10)]. They are plotted in dashed and dotted curves, respectively. Removing the transversality condition has mild effects on the normalized differential rates and little impacts on their values at the resonances.

As shown in Eqs. (2.20) and (2.32), $\eta_{R}$ in these decays are given by

$\eta_{R}=\frac{1}{2} \pi \Gamma_{R} \frac{d \tilde{\Gamma}\left(m_{R}\right)}{d m_{K \pi}}, \quad \eta_{\rho}^{\mathrm{GS}}=\frac{\pi \Gamma_{\rho}^{0}}{2\left(1+D \Gamma_{\rho}^{0} / m_{\rho}\right)^{2}} \frac{d \tilde{\Gamma}\left(m_{\rho}\right)}{d m_{\pi \pi}}$.

From the right plots in Fig. 8, one can read off the values of $\eta_{R}$ from the height of the curves at the resonances. 


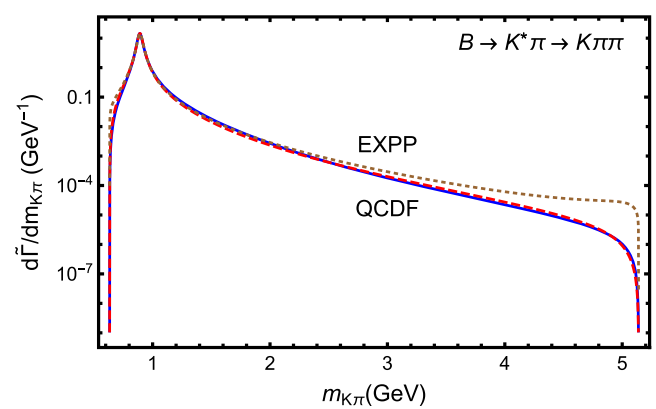

(a)

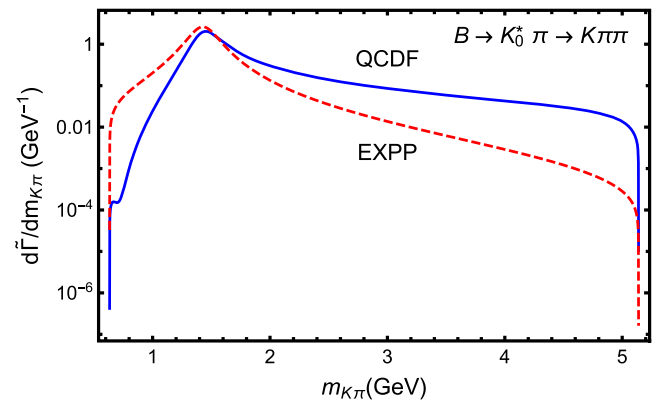

(c)

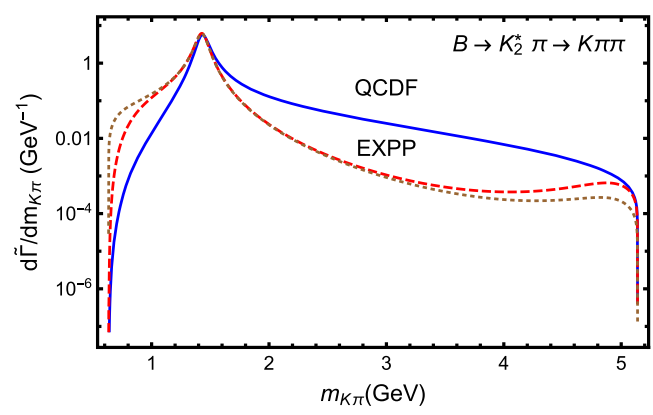

(e)

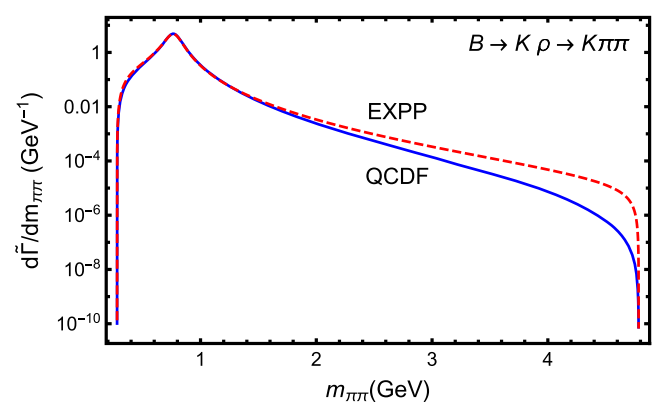

(g)

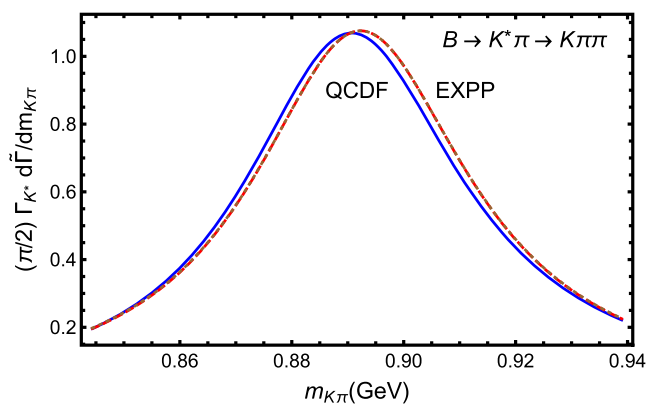

(b)

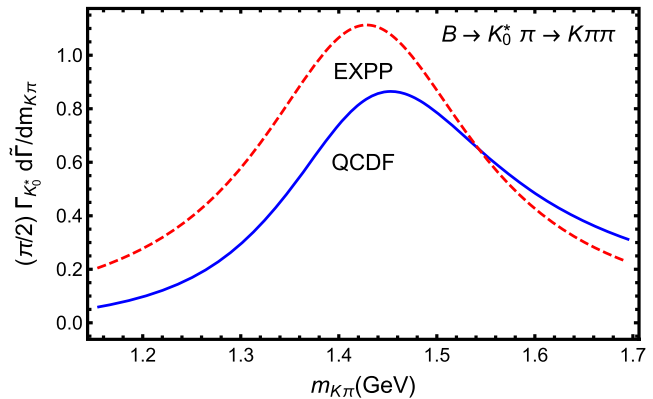

(d)

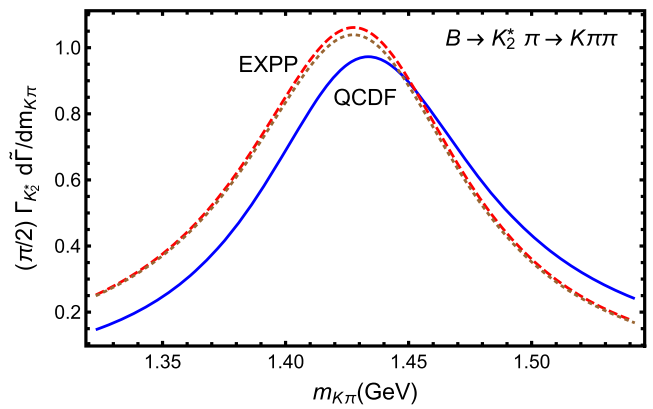

(f)

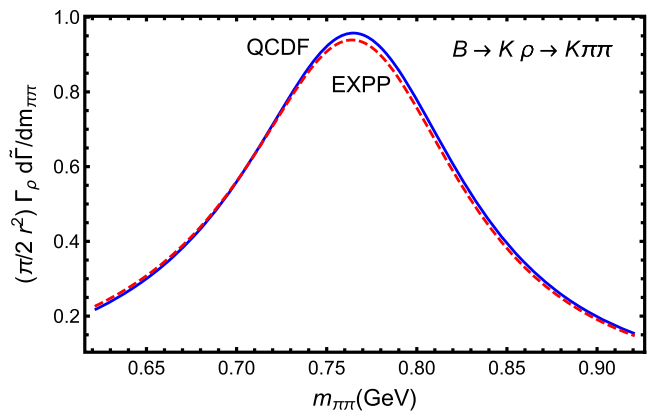

(h)

FIG. 8. Left column: the normalized differential rates in $B^{-} \rightarrow R \pi^{-} \rightarrow K^{-} \pi^{+} \pi^{-}$and $B^{-} \rightarrow K^{-} \rho \rightarrow K^{-} \pi^{+} \pi^{-}$decays. Right column: plots scaled and blown-up in the resonance regions, where the heights at the resonances equal $\eta_{R}$. In plot (h), we use $r \equiv 1+D \Gamma_{\rho}^{0} / m_{\rho}$. The solid curves come from the QCDF calculation and the dashed (dotted) curves from the experimental parametrization with (without) the transversality condition imposed.

The values agree with those shown in Table IV. Recall that for $\Gamma_{R} / m_{R} \ll 1$, we can approximate $\eta_{R}$ by the integration of the normalized differential rate around the resonance as shown in Eq. (2.24). For example, for the $B^{-} \rightarrow R^{0} \pi^{-} \rightarrow K^{-} \pi^{+} \pi^{-}$decays, $\eta_{R}$ can be approximately given by 


$$
\begin{aligned}
\eta_{R} & \simeq \frac{\pi}{2 \tan ^{-1} 2} \int_{m_{R}-\Gamma_{R}}^{m_{R}+\Gamma_{R}} \frac{d \tilde{\Gamma}\left(m_{K \pi}\right)}{d m_{K \pi}} d m_{K \pi} \\
& =\frac{\pi}{2 \tan ^{-1} 2}\left(1-\int_{\text {elsewhere }} \frac{d \tilde{\Gamma}\left(m_{K \pi}\right)}{d m_{K \pi}} d m_{K \pi}\right) .
\end{aligned}
$$

Note that for the case of $\eta_{\rho}^{\mathrm{GS}}$ one needs to include the $1 /\left(1+D \Gamma_{\rho}^{0} / m_{\rho}\right)^{2}$ factor. Numerically, we find that this approximation works well for the decay modes considered in this section. The above equation clearly shows that $\eta_{R}$ represents the fraction of rates around the resonance and it is anticorrelated with the fraction of rates off the resonance.

From Figs. 8(a) and 8(g), we see that for $R=\bar{K}^{* 0}$ and $\rho^{0}$ the normalized differential rates predicted by QCDF are very similar to those obtained by using the experimental parametrization, while for $R=\bar{K}_{0}^{* 0}$ and $\bar{K}_{2}^{* 0}$ the QCDF results and experimental models are different. Consequently, as shown in Figs. 8(b) and 8(h), QCDF and the experimental model give similar values on $d \tilde{\Gamma}\left(m_{\bar{K}^{*}}\right) / d m_{K \pi}$ and $d \tilde{\Gamma}\left(m_{\rho}\right) / d m_{\pi \pi}$, resulting in $\eta_{R}^{\mathrm{QCDF}} \simeq \eta_{R}^{\mathrm{EXPP}}$ for $R=\rho$ and $K^{*}$. In contrast, as shown in Figs. 8(d) and 8(f), the QCDF $d \tilde{\Gamma}\left(m_{\bar{K}_{0}^{*}}\right) / d m_{K \pi}$ and $d \tilde{\Gamma}\left(m_{\bar{K}_{2}}\right) / d m_{K \pi}$ are smaller than those from the experimental model, resulting in $\eta_{K_{0}^{*}, K_{2}^{*}}^{\mathrm{QCDF}}<\eta_{K_{0}^{*}, K_{2}^{*}}^{\mathrm{EXPP}}$.

Using Eq. (5.2), we can relate the smallness of $\eta_{K_{0}^{*}}^{\mathrm{QCDF}}$, comparing to $\eta_{K_{0}^{*}}^{\mathrm{EXP}}$, to the fact that the normalized differential rate obtained in the QCDF calculation is much larger than the one using the experimental parametrization in the off-resonance region, particularly in the large $m_{K \pi}$ region. To verify the source of the enhancement, we note that, as shown in Eq. (4.112), the $m_{K \pi}$ dependence in the QCDF amplitude is governed by the strong decay form factor, $F\left(m_{K \pi}^{2}, m_{K_{0}^{*}}\right)$, the $B \rightarrow \pi$ form factor, $F_{0}^{B \pi}\left(m_{K \pi}^{2}\right)$, and a $m_{K \pi}^{2}$ factors sitting in front of the QCD penguin Wilson coefficient $\left(a_{6}^{p}-a_{8}^{p} / 2\right)$ and related to the so-called chiral factor $\left(r_{\chi}^{S}\right)$ in the two-body decay [see Eq. (4.113)]. The last two factors are responsible for the enhancement of the QCDF differential rate in the large $m_{K \pi}$ region. As shown in
Eq. (3.1) and the equations below it, these two factors are not included in the experimental parametrization for the scalar resonance. As a result, QCDF and the experimental parametrization give different normalized differential rates and $\eta_{R}$ for this mode.

The momentum dependence (such as $m_{K \pi}$ ) of weak dynamics is mode dependent. For example, in the above $B^{-} \rightarrow \bar{K}_{0}^{*}(1430) \pi^{-} \rightarrow K^{-} \pi^{+} \pi^{-}$decay, we have a $m_{K \pi}^{2}$ factor from the chiral factor $r_{\chi}^{S}$, while the chiral factor $r_{\chi}^{V}$ in the $B^{-} \rightarrow \bar{K}^{*}(980) \pi^{-} \rightarrow K^{-} \pi^{+} \pi^{-}$decay does not provide the $m_{K \pi}^{2}$ factor [see Eq. (4.56)]. Such a difference in the momentum dependence of weak dynamics has a visible effect on the shape of the normalized differential rates, as depicted in Figs. 8(a) and 8(c).

As shown in Eq. (3.1), the weak dynamics in the experimental parametrization is basically represented by a complex number, the coefficient $c$, which does not have any momentum dependence. In the narrow width limit, the value of the normalized differential rate is highly dominated by its peak at the resonance, and the values of the normalized differential rate elsewhere cannot compete with it. Therefore, only $m_{K \pi(\pi \pi)} \simeq m_{R}$ matters and, consequently, it is legitimate to use a momentum-independent coefficient, namely, $c$, to represent the weak dynamics. However, in the case of a broad resonance, things are generally different. The peak at the resonance is no longer highly dominating, as its height is affected by the values of the normalized differential rate elsewhere. In this case, the momentum dependence of the weak dynamics cannot be ignored and, hence, using a momentum-independent coefficient to represent the weak dynamics is too naïve.

\section{B. Branching fractions of quasi-two-body decays}

For given experimental measurements of $\mathcal{B}\left(B^{+} \rightarrow\right.$ $R P_{3} \rightarrow P_{1} P_{2} P_{3}$ ), we show in Table $\mathrm{V}$ various branching fractions of the quasi-two-body decays $B^{+} \rightarrow R P_{3}$. $\mathcal{B}\left(B^{+} \rightarrow R P_{3}\right)_{\mathrm{NWA}}$ denotes the branching fraction obtained from Eq. (1.2) in the NWA. Our results of $\mathcal{B}\left(B^{+} \rightarrow\right.$ $\left.R P_{3}\right)_{\mathrm{NWA}}$ for $B^{+} \rightarrow K_{2}^{* 0}(1430) \pi^{+}, K^{* 0} \pi^{+}$, and $K^{+} \rho^{0}$

TABLE V. Branching fractions of quasi-two-body decays $B^{+} \rightarrow R P_{3}$ (in units of $10^{-6}$ ) derived from the measured $B^{+} \rightarrow R P_{3} \rightarrow$ $P_{1} P_{2} P_{3}$ rates. $\mathcal{B}\left(B^{+} \rightarrow R P_{3}\right)_{\mathrm{NWA}}$ denotes the branching fraction obtained from Eq. (1.2) in the narrow width approximation.

\begin{tabular}{lcccc}
\hline \hline Mode & $\mathcal{B}\left(B^{+} \rightarrow R P_{3} \rightarrow P_{1} P_{2} P_{3}\right)_{\mathrm{expt}}$ & $\mathcal{B}\left(B^{+} \rightarrow R P_{3}\right)_{\mathrm{NWA}}$ & $\eta_{R}^{\mathrm{QCDF}} \mathcal{B}\left(B^{+} \rightarrow R P_{3}\right)_{\mathrm{NWA}}$ & $\eta_{R}^{\mathrm{EXPP}} \mathcal{B}\left(B^{+} \rightarrow R P_{3}\right)_{\mathrm{NWA}}$ \\
\hline$B^{+} \rightarrow f_{2} \pi^{+} \rightarrow \pi^{+} \pi^{-} \pi^{+}$ & $1.17 \pm 0.20[8-10]$ & $2.08 \pm 0.36$ & $2.09 \pm 0.36$ & $1.95 \pm 0.33$ \\
$B^{+} \rightarrow K_{2}^{* *} \pi^{+} \rightarrow K^{+} \pi^{-} \pi^{+}$ & $1.85_{-0.50}^{+0.73}[12,30]$ & $5.56_{-1.50}^{+2.19}$ & $5.40_{-1.46}^{+2.13}$ & $5.85_{-1.58}^{+2.31}$ \\
$B^{+} \rightarrow \rho^{0} \pi^{+} \rightarrow \pi^{+} \pi^{-} \pi^{+}$ & $8.36 \pm 0.77[8-10]$ & $8.36 \pm 0.77$ & $7.78 \pm 0.72(\mathrm{GS})$ & $7.95 \pm 0.73$ (GS) \\
& & & $9.28 \pm 0.86(\mathrm{BW})$ & \\
$B^{+} \rightarrow K^{+} \rho^{0} \rightarrow K^{+} \pi^{+} \pi^{-}$ & $3.7 \pm 0.5[12,30]$ & $3.7 \pm 0.5$ & $3.5 \pm 0.5(\mathrm{GS})$ & $3.4 \pm 0.5(\mathrm{GS})$ \\
& & & $4.2 \pm 0.6(\mathrm{BW})$ & \\
$B^{+} \rightarrow K^{* 0} \pi^{+} \rightarrow K^{+} \pi^{-} \pi^{+}$ & $6.71 \pm 0.57[12,30]$ & $10.1 \pm 0.8$ & $10.7 \pm 0.9$ & $10.9 \pm 0.9$ \\
$B^{+} \rightarrow \sigma \pi^{+} \rightarrow \pi^{+} \pi^{-} \pi^{+}$ & $3.83 \pm 0.84[9,10]$ & $5.75 \pm 1.26$ & $12.36 \pm 2.71$ & $9.44 \pm 2.08$ \\
$B^{+} \rightarrow K_{0}^{* 0} \pi^{+} \rightarrow K^{+} \pi^{-} \pi^{+}$ & $27.9_{-4.3}^{+5.6}[12,30]$ & $45_{-7}^{+9}$ & $37_{-6}^{+8}$ & $50_{-8}^{+10}$ \\
\hline \hline
\end{tabular}


modes agree with the PDG data [3]. For $B^{+} \rightarrow$ $f_{2}(1270) \pi^{+}, \rho^{0} \pi^{+}$, and $\sigma \pi^{+}$decays, we have included the new measurement of $B^{+} \rightarrow \pi^{+} \pi^{+} \pi^{-}$performed by the LHCb Collaboration $[9,10]$. As for $\mathcal{B}\left(B^{+} \rightarrow K_{0}^{* 0} \pi^{+}\right)_{\mathrm{NWA}}$, our value is different from $\left(39_{-5}^{+6}\right) \times 10^{-6}$ given by PDG [3] as the contribution of $B^{+} \rightarrow K_{0}^{* 0} \pi^{+} \rightarrow K_{S}^{0} \pi^{0} \pi^{+}$[38] is included in the latter case.

When the resonance is sufficiently broad, it is necessary to take into account the finite-width effects characterized by the parameter $\eta_{R}$. In Table $\mathrm{V}$, we have shown the corrections to $\mathcal{B}\left(B^{+} \rightarrow R P_{3}\right)_{\mathrm{NWA}}$ in both QCDF and EXPP schemes. Although the finite-width effects are generally small, they are significant in the $B^{+} \rightarrow \rho \pi^{+}$decay and prominent in $B^{+} \rightarrow \sigma / f_{0}(500) \pi^{+}$and $B^{+} \rightarrow K_{0}^{* 0}(1430) \pi^{+}$. For example, the PDG value of $\mathcal{B}\left(B^{+} \rightarrow \rho \pi^{+}\right)=(8.3 \pm 1.2) \times 10^{-6}$ [3] should be corrected to $(7.7 \pm 1.1) \times 10^{-6}$ in QCDF or $(7.9 \pm 1.1) \times 10^{-6}$ in EXPP. The large width effects in the $\sigma / f_{0}(500)$ production imply that $B^{-} \rightarrow \sigma \pi^{-}$has a large branching fraction of order $10^{-5}$. More precisely, the LHCb value of $\mathcal{B}\left(B^{+} \rightarrow \sigma \pi^{+}\right)=(5.8 \pm 1.3) \times 10^{-6}$ should be corrected to $(12.4 \pm 2.7) \times 10^{-6}$ in QCDF or $(9.4 \pm 2.1) \times$ $10^{-6}$ in EXPP.

\section{CONCLUSIONS}

For the branching fractions of the quasi-two-body decays $\mathcal{B}\left(B \rightarrow R P_{3}\right)$ with $R$ being an intermediate resonant state, it is a common practice to apply the factorization relation, also known as the NWA, to extract them from the measured process $B \rightarrow R P_{3} \rightarrow P_{1} P_{2} P_{3}$. However, such a treatment is valid only in the narrow width limit of the intermediate resonance, namely, $\Gamma_{R} \rightarrow 0$. In this work, we have studied the corrections to $\mathcal{B}\left(B \rightarrow R P_{3}\right)$ arising from the finite-width effects. We consider the parameter $\eta_{R}$ which is the ratio of the three-body decay rate without and with the finite-width effects of the resonance. Our main results are as follows:

(i) We have presented a general framework for the parameter $\eta_{R}$ and shown that it can be expressed in terms of the normalized differential rate and is determined by its value evaluated at the resonance. Since the value of the normalized differential rate at the resonance is anticorrelated with the normalized differential rate off the resonance, it is the shape of the normalized differential rate that matters in the determination of $\eta_{R}$.

(ii) In the experimental analysis of $B \rightarrow R P_{3} \rightarrow P_{1} P_{2} P_{3}$ decays, it is customary to parametrize the amplitude as $A\left(m_{12}, m_{23}\right)=c F\left(m_{12}, m_{23}\right)$, where the strong dynamics is described by the function $F$ parametrized in terms of the resonance line shape, the angular dependence, and Blatt-Weisskopf barrier factors, while the information of weak interactions is encoded in the complex coefficients $c$. We evaluate $\eta_{R}$ in this experimentally motivated parametrization and in the theoretical framework of QCDF. (iii) In QCDF calculations, we have verified the NWA relation both analytically and numerically for some charged $B$ decays involving tensor, vector, and scalar resonances. We have introduced a form factor $F\left(s_{12}, m_{R}\right)$ for the strong coupling of $R\left(m_{12}\right) \rightarrow$ $P_{1} P_{2}$ when $m_{12}$ is away from $m_{R}$. We find that offshell effects are small in vector meson productions, but prominent in the $K_{2}^{*}(1430), \sigma / f_{0}(500)$, and $K_{0}^{*}(1430)$ resonances.

(iv) In principle, the two-body rates reported by experiments should be corrected using $\eta_{R}=\eta_{R}^{\mathrm{EXPP}}$ in Eq. (1.4), as the data are extracted using the experimental parametrization. On the other hand, the experimental parametrization of the normalized differential rates should be compared with the theoretical predictions using QCDF calculations as the latter take into account the energy dependence of weak interaction amplitudes. In some cases, where $\eta_{R}^{\mathrm{EXPP}}$ are very different from $\eta_{R}^{\mathrm{QCDF}}$, we note that using an energy-independent coefficient $c$, in the experimental parametrization, to represent the weak dynamics is too naïve. Moreover, systematic uncertainties in these experimental results after being corrected by $\eta_{R}^{\mathrm{EXPP}}$ are still underestimated.

(v) We have compared between $\eta_{R}^{\mathrm{QCDF}}$ and $\eta_{R}^{\mathrm{EXPP}}$ for their width dependence in Figs. 1-7. Numerical results are summarized in Table IV. In general, the two quantities are similar for vector mesons but different for tensor and scalar mesons. A study of the differential rates in Fig. 8 enables us to understand the origin of their differences. For example, the similar normalized differential rates for $\rho$ and $K^{*}$ at and near the resonance account for $\eta_{\rho, K^{*}}^{\mathrm{QCDF}} \simeq \eta_{\rho, K^{*}}^{\mathrm{EXP}}$. In contrast, the $m_{K \pi}^{2}$ dependence associated with the penguin Wilson coefficients $\left(a_{6}^{p}-a_{8}^{p} / 2\right)$ in $B^{-} \rightarrow$ $\bar{K}_{0}^{*}(1430) \pi^{-} \rightarrow K^{-} \pi^{+} \pi^{+}$yields a large enhancement in the QCDF differential rate in the large $m_{K \pi}$ distribution, rendering $\eta_{K_{0}^{*}}^{\mathrm{QCDF}}<\eta_{K_{0}^{*}}^{\mathrm{EXP}}$.

(vi) Finite-width corrections to $\mathcal{B}\left(B^{+} \rightarrow R P\right)_{\mathrm{NWA}}$, the branching fractions of quasi-two-body decays obtained in the NWA, are summarized in Table V for both QCDF and EXPP schemes. In general, finitewidth effects are small, less than $10 \%$, but they are prominent in $B^{+} \rightarrow \sigma / f_{0}(500) \pi^{+}$and $B^{+} \rightarrow$ $K_{0}^{* 0}(1430) \pi^{+}$decays.

(vii) It is customary to use the Gounaris-Sakurai model to describe the line shape of the broad $\rho(770)$ resonance to ensure the unitarity far from the pole mass. If the relativistic Breit-Wigner model is employed instead, we find $\eta_{\rho}^{\mathrm{BW}}>1>\eta_{\rho}^{\mathrm{GS}}$ in both QCDF and EXPP schemes owing to the $\left(1+D \Gamma_{\rho}^{0} / m_{\rho}\right)$ term in the GS model. For example, in the presence of finitewidth corrections, the PDG value of $\mathcal{B}\left(B^{+} \rightarrow\right.$ $\left.\rho \pi^{+}\right)=(8.3 \pm 1.2) \times 10^{-6}$ should be corrected to 
$(7.7 \pm 1.1) \times 10^{-6}$ in $\mathrm{QCDF}$ and $(7.9 \pm 1.1) \times 10^{-6}$ in EXPP.

(viii) The $\sigma / f_{0}(500)$ scalar resonance is very broad, and its line shape cannot be described by the familiar Breit-Wigner model. We have followed the $\mathrm{LHCb}$ Collaboration to use a simple pole model description. We have found very large width effects: $\eta_{\sigma}^{\mathrm{QCDF}} \sim 2.15$ and $\eta_{\sigma}^{\mathrm{EXPP}} \sim 1.64$. Consequently, $B^{-} \rightarrow \sigma \pi^{-}$has a large branching fraction of order $10^{-5}$.

(ix) We have employed the Breit-Wigner line shape to describe the production of $K_{0}^{*}(1430)$ in three-body $B$ decays and found large off-shell effects. The smallness of $\eta_{K_{0}^{*}}^{\mathrm{QCDF}}$ relative to $\eta_{K_{0}^{*}}^{\mathrm{EXPP}}$ is ascribed to the fact that the normalized differential rate obtained in the QCDF calculation is much larger than that using the
EXPP scheme in the off-resonance region. The large discrepancy between QCDF estimate and experimental data of $\Gamma\left(B^{-} \rightarrow \bar{K}_{0}^{* 0} \pi^{-} \rightarrow K^{-} \pi^{+} \pi^{-}\right)$still remains an enigma.

(x) In the approach of QCDF, the calculated $C P$ asymmetries of $B^{-} \rightarrow f_{2}(1270) \pi^{-}, B^{-} \rightarrow \sigma / f_{0}(500) \pi^{-}$, and $B^{-} \rightarrow K^{-} \rho^{0}$ agree with the experimental observations. The nonobservation of $C P$ asymmetry in $B^{-} \rightarrow \rho(770) \pi^{-}$can also be accommodated in QCDF.

\section{ACKNOWLEDGMENTS}

This research was supported in part by the Ministry of Science and Technology of R.O.C. under Grants No. MOST-106-2112-M-033-004-MY3 and No. MOST108-2112-M-002-005-MY3.
[1] H. Y. Cheng, Comments on the quark content of the scalar meson $f_{0}(1370)$, Phys. Rev. D 67, 054021 (2003).

[2] T. Huber, J. Virto, and K. K. Vos, Three-body non-leptonic heavy-to-heavy $B$ decays at NNLO in QCD, J. High Energy Phys. 11 (2020) 103.

[3] P. A. Zyla et al. (Particle Data Group), Review of particle physics, Prog. Theor. Exp. Phys. (2020), 083C01.

[4] H. Y. Cheng, Hadronic charmed meson decays involving axial vector mesons, Phys. Rev. D 67, 094007 (2003).

[5] H. Y. Cheng, C. W. Chiang, and C. K. Chua, Width effects in resonant three-body $B$ decays, Phys. Lett. B 813, 136058 (2021).

[6] D. Asner, Charm Dalitz plot analysis formalism and results: Expanded RPP-2004 version, arXiv:hep-ex/0410014.

[7] G. J. Gounaris and J. J. Sakurai, Finite-Width Corrections to the Vector Meson Dominance Prediction for $\rho \rightarrow e^{+} e^{-}$, Phys. Rev. Lett. 21, 244 (1968).

[8] B. Aubert et al. (BABAR Collaboration), Dalitz plot analysis of $B^{+} \rightarrow \pi^{+} \pi^{+} \pi^{-}$decays, Phys. Rev. D 79, 072006 (2009).

[9] R. Aaij et al. (LHCb Collaboration), Observation of Several Sources of $C P$ Violation in $B^{+} \rightarrow \pi^{+} \pi^{+} \pi^{-}$Decays, Phys. Rev. Lett. 124, 031801 (2020).

[10] R. Aaij et al. (LHCb Collaboration), Amplitude analysis of the $B^{+} \rightarrow \pi^{+} \pi^{+} \pi^{-}$decay, Phys. Rev. D 101, 012006 (2020).

[11] J. R. Pelaez, From controversy to precision on the sigma meson: A review on the status of the non-ordinary $f_{0}(500)$ resonance, Phys. Rep. 658, 1 (2016).

[12] B. Aubert et al. (BABAR Collaboration), Evidence for direct $C P$ violation from Dalitz-plot analysis of $B^{ \pm} \rightarrow K^{ \pm} \pi^{\mp} \pi^{ \pm}$, Phys. Rev. D 78, 012004 (2008).

[13] M. Beneke, G. Buchalla, M. Neubert, and C. T. Sachrajda, QCD Factorization for $B \rightarrow P P$ Decays: Strong Phases and $C P$ Violation in the Heavy Quark Limit, Phys. Rev. Lett. 83, 1914 (1999); QCD factorization for exclusive, nonleptonic
B meson decays: General arguments and the case of heavy light final states, Nucl. Phys. B591, 313 (2000).

[14] M. Beneke and M. Neubert, QCD factorization for $B \rightarrow P P$ and $B \rightarrow P V$ decays, Nucl. Phys. B675, 333 (2003).

[15] H. Y. Cheng and C. K. Chua, Branching fractions and $C P$ violation in $B^{-} \rightarrow K^{+} K^{-} \pi^{-}$and $B^{-} \rightarrow \pi^{+} \pi^{-} \pi^{-}$decays, Phys. Rev. D 102, 053006 (2020).

[16] H. Y. Cheng and K. C. Yang, Charmless hadronic $B$ decays into a tensor meson, Phys. Rev. D 83, 034001 (2011).

[17] H. Y. Cheng, C. K. Chua, and A. Soni, Charmless threebody decays of $B$ mesons, Phys. Rev. D 76, 094006 (2007).

[18] H. Y. Cheng and C. K. Chua, Branching fractions and direct $C P$ violation in charmless three-body decays of $B$ mesons, Phys. Rev. D 88, 114014 (2013).

[19] H. Y. Cheng, C. K. Chua, and Z. Q. Zhang, Direct $C P$ violation in charmless three-body decays of $B$ mesons, Phys. Rev. D 94, 094015 (2016).

[20] W. Wang, B to tensor meson form factors in the perturbative QCD approach, Phys. Rev. D 83, 014008 (2011).

[21] H. Y. Cheng, C. K. Chua, and C. W. Hwang, Covariant light front approach for $\mathrm{s}$ wave and $\mathrm{p}$ wave mesons: Its application to decay constants and form-factors, Phys. Rev. D 69, 074025 (2004).

[22] J. P. Dedonder, A. Furman, R. Kaminski, L. Lesniak, and B. Loiseau, $S$-, $P$ - and $D$-wave final state interactions and $C P$ violation in $B^{ \pm} \rightarrow \pi^{ \pm} \pi^{\mp} \pi^{ \pm}$decays, Acta Phys. Pol. B 42, 2013 (2011).

[23] H. Y. Cheng, C. K. Chua, and A. Soni, Final state interactions in hadronic $B$ decays, Phys. Rev. D 71, 014030 (2005).

[24] J. H. Alvarenga Nogueira, I. Bediaga, A. B. R. Cavalcante, T. Frederico, and O. Lourenmo, $C P$ violation: Dalitz interference, $C P T$, and final state interactions, Phys. Rev. D 92, 054010 (2015). 
[25] H. Y. Cheng, Y. Koike, and K. C. Yang, Two-parton lightcone distribution amplitudes of tensor mesons, Phys. Rev. D 82, 054019 (2010).

[26] J. Tandean and S. Gardner, Nonresonant contributions in $B \rightarrow \rho \pi$ decay, Phys. Rev. D 66, 034019 (2002); S. Gardner and U. G. Meissner, Rescattering and chiral dynamics in $B \rightarrow \rho \pi$ decay, Phys. Rev. D 65, 094004 (2002).

[27] M. Wirbel, B. Stech, and M. Bauer, Exclusive semileptonic decays of heavy mesons, Z. Phys. C 29, 637 (1985); M. Bauer, B. Stech, and M. Wirbel, Exclusive nonleptonic decays of $D, D_{s}$, and $B$ mesons, Z. Phys. C 34, 103 (1987).

[28] H. Y. Cheng, $C P$ violation in $B^{ \pm} \rightarrow \rho^{0} \pi^{ \pm}$and $B^{ \pm} \rightarrow \sigma \pi^{ \pm}$ decays, arXiv:2005.06080.

[29] H. Y. Cheng and C. K. Chua, Revisiting charmless hadronic $B_{u, d}$ decays in QCD factorization, Phys. Rev. D 80, 114008 (2009).

[30] A. Garmash et al. (Belle Collaboration), Evidence for Large Direct $C P$ Violation in $B^{ \pm} \rightarrow \rho(770)^{0} K^{ \pm}$from Analysis of the Three-Body Charmless $B^{ \pm} \rightarrow K^{ \pm} \pi^{ \pm} \pi^{\mp}$ Decay, Phys. Rev. Lett. 96, 251803 (2006).

[31] J. J. Qi, Z. Y. Wang, X. H. Guo, and Z. H. Zhang, Study of localized $C P$ violation in $B^{-} \rightarrow \pi^{-} \pi^{+} \pi^{-}$and the branching ratio of $B^{-} \rightarrow \sigma(600) \pi^{-}$in the QCD factorization approach, Nucl. Phys. B948, 114788 (2019).
[32] H. Y. Cheng, C. K. Chua, and K. C. Yang, Charmless hadronic $B$ decays involving scalar mesons: Implications to the nature of light scalar mesons, Phys. Rev. D 73, 014017 (2006).

[33] R. Aaij et al. (LHCb Collaboration), Dalitz plot analysis of $B^{0} \rightarrow \bar{D}^{0} \pi^{+} \pi^{-}$decays, Phys. Rev. D 92, 032002 (2015).

[34] D. V. Bugg, The mass of the sigma pole, J. Phys. G 34, 151 (2007).

[35] H. Y. Cheng, C. K. Chua, K. C. Yang, and Z. Q. Zhang, Revisiting charmless hadronic $B$ decays to scalar mesons, Phys. Rev. D 87, 114001 (2013).

[36] B. El-Bennich, A. Furman, R. Kaminski, L. Lesniak, B. Loiseau, and B. Moussallam, $C P$ violation and kaonpion interactions in $B \rightarrow K \pi^{+} \pi^{-}$decays, Phys. Rev. D 79, 094005 (2009); Erratum, Phys. Rev. D 83, 039903 (2011).

[37] D. Aston, N. Awaji, T. Bienz, F. Bird, J. D'Amore, W. Dunwoodie, R. Endorf, K. Fujii, H. Hayashi and S. Iwata et al. A study of $K^{-} \pi^{+}$scattering in the reaction $K^{-} p \rightarrow$ $K^{-} \pi^{+} n$ at $11-\mathrm{GeV} / \mathrm{c}, \mathrm{Nucl}$. Phys. B296, 493 (1988).

[38] J. P. Lees et al. (BABAR Collaboration), Evidence for $C P$ violation in $B^{+} \rightarrow K^{*}(892)^{+} \pi^{0}$ from a Dalitz plot analysis of $B^{+} \rightarrow K_{\mathrm{S}}^{0} \pi^{+} \pi^{0}$ decays, Phys. Rev. D 96, 072001 (2017). 